\title{
DESIGN OF MEMS MAGNETIC ACTUATOR FOR MEMS FOURIER TRANSFORM INFRARED SPECTROMETER
}

\author{
by \\ Farzana Husain \\ Bachelor of Mechanical Engineering, Bangladesh University of Engineering and Technology, \\ 2004
}

\author{
A thesis \\ presented to Ryerson University \\ in partial fulfillment of the \\ requirements for the degree of \\ Master of Applied Science \\ in the Program of \\ Mechanical and Industrial Engineering
}

Toronto, Ontario, Canada, 2013

(c) Farzana Husain 2013 


\section{Author's declaration}

I hereby declare that I am the sole author of this thesis. This is a true copy of the thesis, including any required final revisions, as accepted by my examiners.

I authorize Ryerson University to lend this thesis to other institutions or individuals for the purpose of scholarly research

I further authorize Ryerson University to reproduce this thesis by photocopying or by other means, in total or in part, at the request of other institutions or individuals for the purpose of scholarly research.

I understand that my thesis may be made electronically available to the public. 
Title of thesis: Design of MEMS Magnetic Actuator for MEMS Fourier Transform Infrared Spectrometer

Name: Farzana Husain

Master of Applied Science in the Program of Mechanical Engineering, Ryerson University, 2013

\begin{abstract}
The objective of this thesis is to design MEMS magnetic actuator for MEMS FTIRS. The actuator consists of moving part and fixed part. The moving part uses rotation-to-translation motion conversion mechanism to achieve large translation, which includes four trapezoidal plates, central ring, anchoring springs and connection springs. The fixed part of the actuator consists of four solenoids. The actuator can be integrated with separately fabricated micromirror plate to achieve high surface quality translation micromirror for FTIRS. The actuator is capable of eliminating titling by controlling the four solenoids individually. The MEMS magnetic actuator has been designed and simulated to be able to output a static displacement of 370micrometers. The stress has been analyzed for the moving part of the actuator. The actuator fixed part has been designed. Dynamic analysis has been conducted for the moving part of the actuator. The moving part of the actuator has been fabricated using MetalMUMPs.
\end{abstract}




\section{Acknowledgements}

I would like to thank:

* My supervisor, Dr. Siyuan He, for his encouragement, support and guidance in this research project

* Yuan Xue, Kenneth Tam, Chao Fan, Zewdu Hailu for being great graduate friends throughout my time at Ryerson University

* My family for their love, support and encouragements

* Special thanks to Muntazera Husain for proofreading the thesis

* The following groups for their collaboration and financial support on this project:

O Ryerson University

o CMC Microsystems 


\section{Table of Contents}

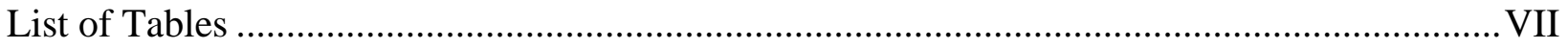

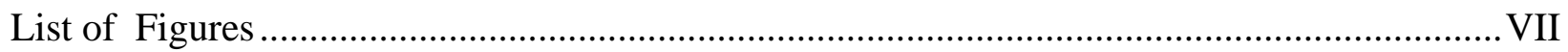

Chapter 1 Introduction ......................................................................................................... 1

1.1The current technology and problems for alcohol interlock ................................................. 2

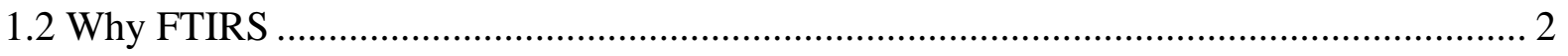

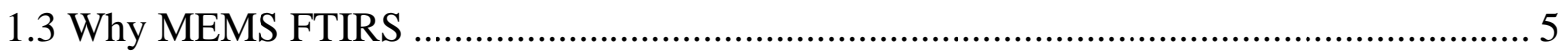

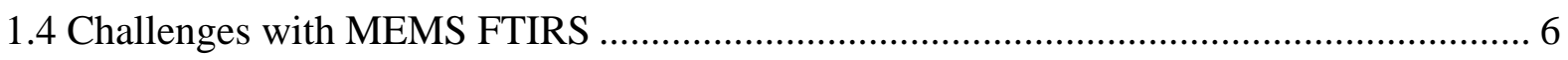

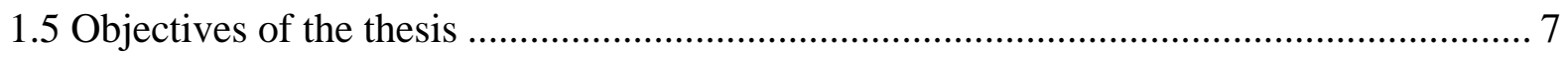

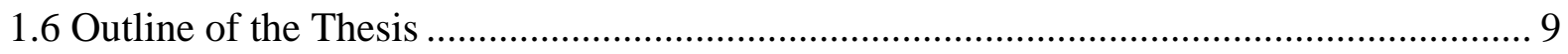

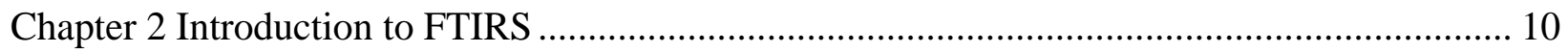

2.1 The Sample Analysis Process ............................................................................................ 10

2.2 Introduction to MEMS FTIRS ………………………................................................... 12

2.2.1 Different design approach of MEMS based FTIRS: ................................................. 12

2.2.2 MEMS FTIRS with high sensitivity ...................................................................... 15

2.3MEMS magnetic actuators ............................................................................................ 18

Chapter 3 Actuator and Translation Micromirror ……………………………………………..... 23

3.1 Magnetic driven micromirror for FTIRS: ........................................................................ 23

3.2 Working principle of the magnetic actuator........................................................................ 25

3.3 Translation Micromirror................................................................................................. 30

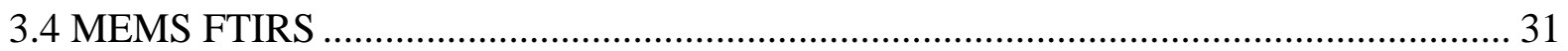

3.5 Minimizing Tilt …………………………………................................................... 32

Chapter 4: Design of Actuator ................................................................................................... 34

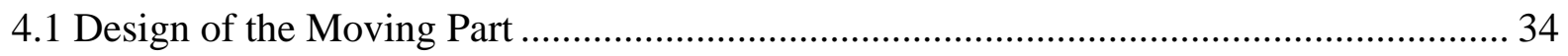




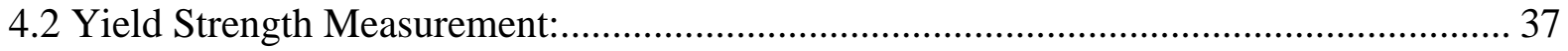

4.3 Simulated Performances of the Actuator ................................................................ 39

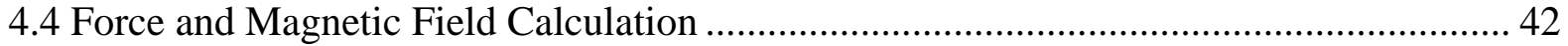

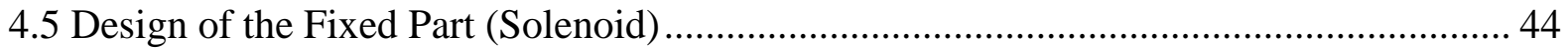

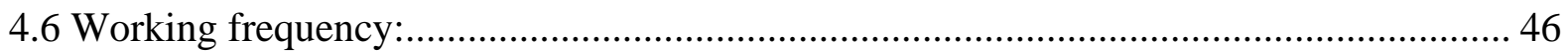

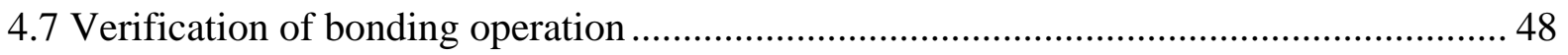

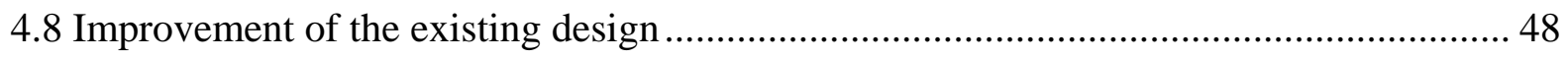

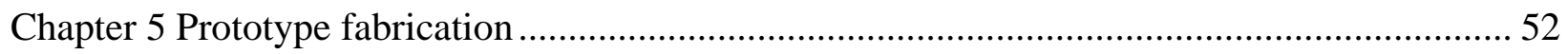

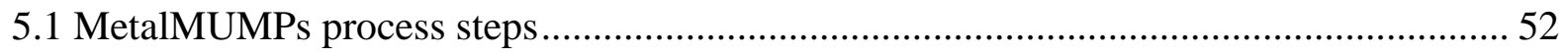

5.2 MetalMUMPs layers and materials properties...................................................... 55

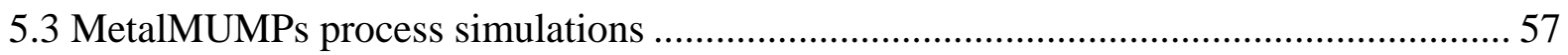

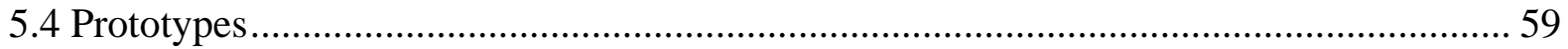

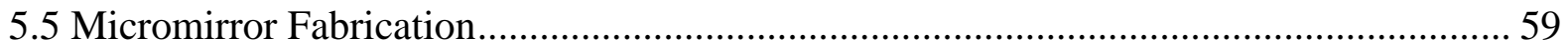

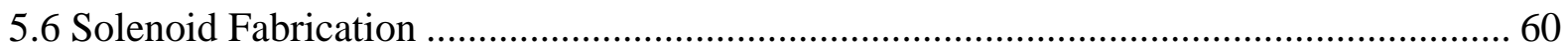

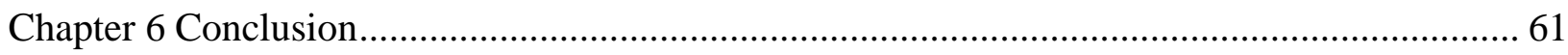

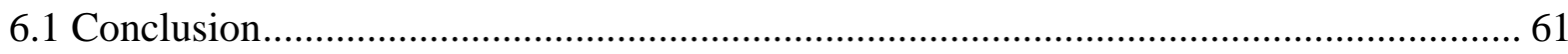

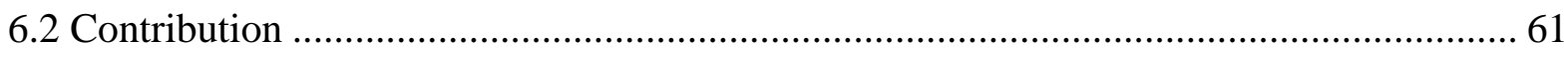

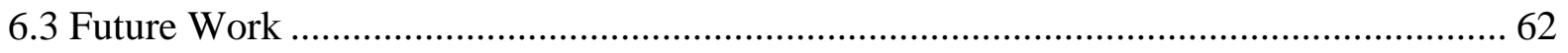

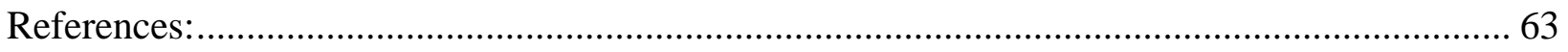




\section{List of Tables}

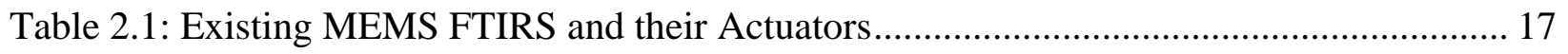

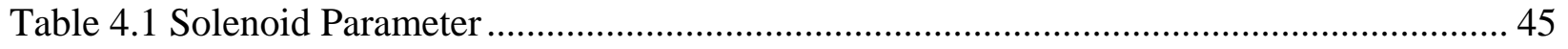

Table 4.2: First Three Mode of Natural Frequency …........................................................ 47

Table 5.1: Layer names, thicknesses and lithography levels (from MetalMUPs hand book) ...... 56

Table 5.2: MEMSCAP level name, CIF and GDSII ${ }^{\mathrm{TM}}$ level designation, and nominal and

minimum features and spaces for each level.(MetalMUMPs Manual) .................................... 57 


\section{List of Figures}

Figure 1.1: Available Alcohol Interlock System for Automobile.......................................... 1

Figure 1.2: Schematic of Dispersive Spectrometer.............................................................. 3

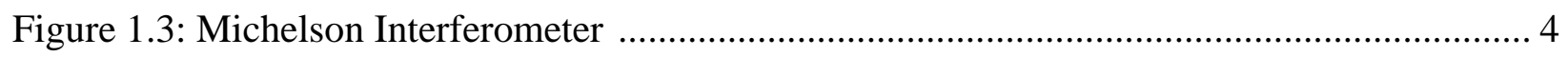

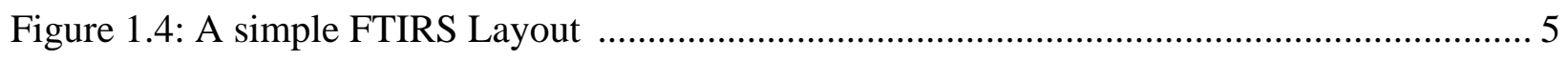

Figure 1.5: Use of Designed Actuator in MEMS FTIRS ..................................................... 8

Figure 2.1: Schematic diagram of sample analysis process using FTIRS ............................... 10

Figure 2.2: Layout of Michelson Interferometer based FTIRS ............................................ 13

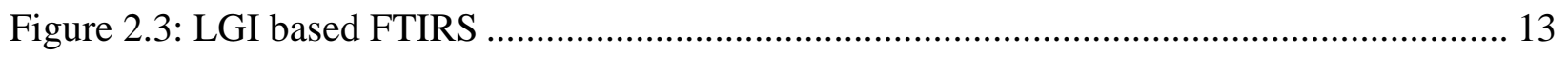

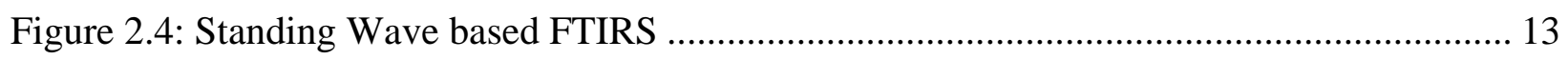

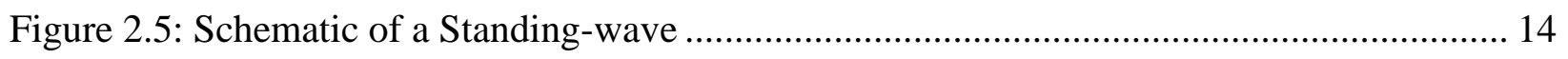

Figure 2.6: (a) MEMS Michelson interferometer by Block MEMS, (b) SEM Micrograph ....... 16

Figure 2.7: Schematic Design of the Mirror Plate and Basic Pantograph Suspension Designed by

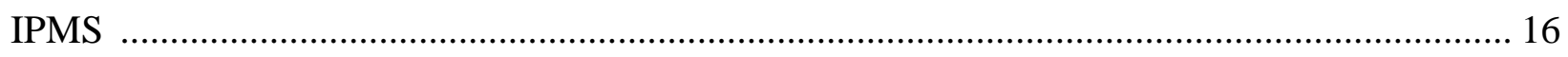

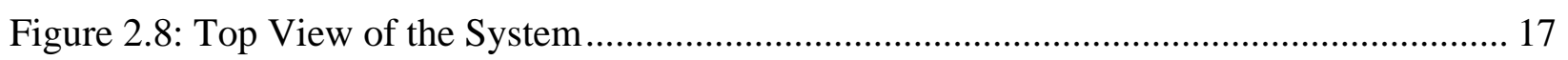

Figure 2.9: Structure of a cantilever beam microactuator: (a) schematic view and (b) cut view...

Figure 2.10: Schematic diagram of two-axis electromagnetically actuated micromirror............. 19

Figure 2.11: Sketch for the construction of the fabricated electromagnetic actuator ................. 20

Figure 2.12: Schematic diagram of three-axis electromagnetically actuated micromirror........... 21

Figure 2.13: Schematic of the electromagnetic actuator. (a) Overhead view of the micro actuator.

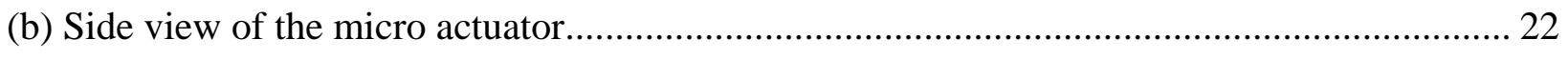

Figure 2.14 MEMS Antenna Driven by Electromagnetic Actuator ....................................... 22 
Figure 3.1: Magnetic Driven Micromirror Assembly.

Figure 3.2: Magnetic Actuation ................................................................................ 26

Figure 3.3: Magnetization and Force States in Magnetic Field ............................................ 27

Figure 3.4: Illustration of Actuation Principle. (a) Rest Position, (b) Out of Plane Actuation due

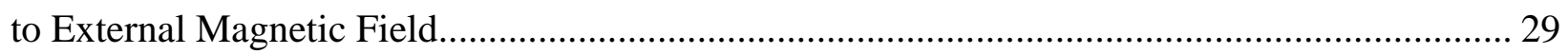

Figure 3.5: (a) Micromirror, (b) Actuator, (c) Translation Micromirror after Bonding .............. 30

Figure 4.1: 2D Diagram of Proposed Magnetic Actuator...................................................... 35

Figure 4.2: Detail design of (a) Trapezoidal Plate, (b) Central Ring, (c) Anchoring Spring, (d)

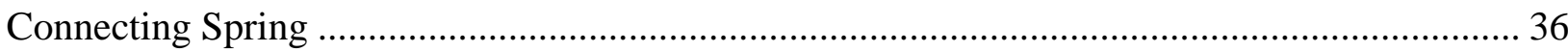

Figure 4.3: Typical yield behaviour for non-ferrous alloys. 1: True elastic limit, 2:

Proportionality limit, 3: Elastic limit, 4: Offset yield strength ........................................... 37

Figure 4.4: Yield Stress Measurement by Sharpe et al. , 2002 ............................................ 38

Figure 4.5: Measurement of Yield Stress for Electroplated Nickel ....................................... 38

Figure 4.6: Simulated Force vs. Displacement ................................................................ 39

Figure 4.7: Simulated Force vs. VonMises Stress .......................................................... 40

Figure 4.8: Displacement of Actuator @ 5mN Load................................................................ 41

Figure 4.9: VonMises Stress of Actuator @ 5mN Load........................................................... 41

Figure 4.10: Actuator and Solenoid arrangement and its cross-sectional view of a single

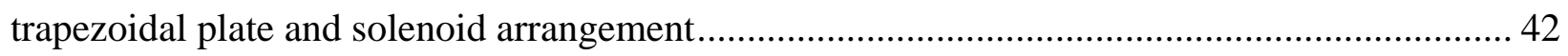

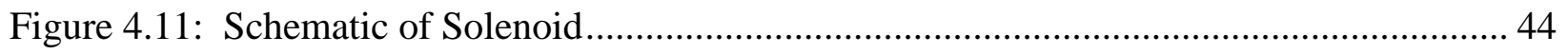

Figure 4.12: First mode of natural frequency (without mirror) .......................................... 47

Figure 4.13: First mode of natural frequency(with mirror) .............................................. 48 
Figure 4.14: Stress Concentration at the Corner of the Spring: (a) Anchoring Spring, (b)

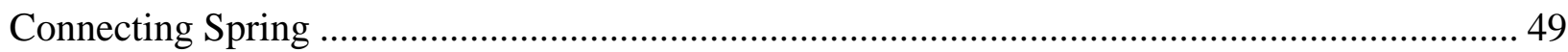

Figure 4.15: Layout of Springs (a) Box Spring, (b) Serpentine Spring .................................. 50

Figure 5.1: Graphical Presentation of MetalMUMPs Process .............................................. 55

Figure 5.2: Actuator Design in AUTOCAD ...................................................................... 58

Figure 5.3: MetalMUMPs simulation of creating 3D model using Coventorware software....... 59

Figure 5.4: Protoype under Microscope Zygo ................................................................... 60 


\section{Chapter 1: Introduction}

Alcohol interlock is a breath testing device which is installed in a vehicle to prevent drunk driving. It is connected to the starter or ignition system of the vehicle which requires the breath sampling of the driver before starting a vehicle. This device will prevent the vehicle from starting if the driver's breath alcohol concentration (BAC) exceeds a preset level (usually corresponding to blood alcohol concentration of 0.02)[1]. Once a breath sample has been provided, and if the amount of alcohol in the breath sample exceeds the recommended level, the alcohol interlock interrupts the flow of power between the vehicle ignition and the starter system of the engine. This device also requires the driver to continue to pass repeated breath tests while the vehicle is in use to ensure that the driver remains sober. The alcohol interlock will never interfere with the running engine as this device is connected to the starter system and not to the engine of the vehicle [2]. Images of available alcohol antilock device are shown in Fig 1.1.
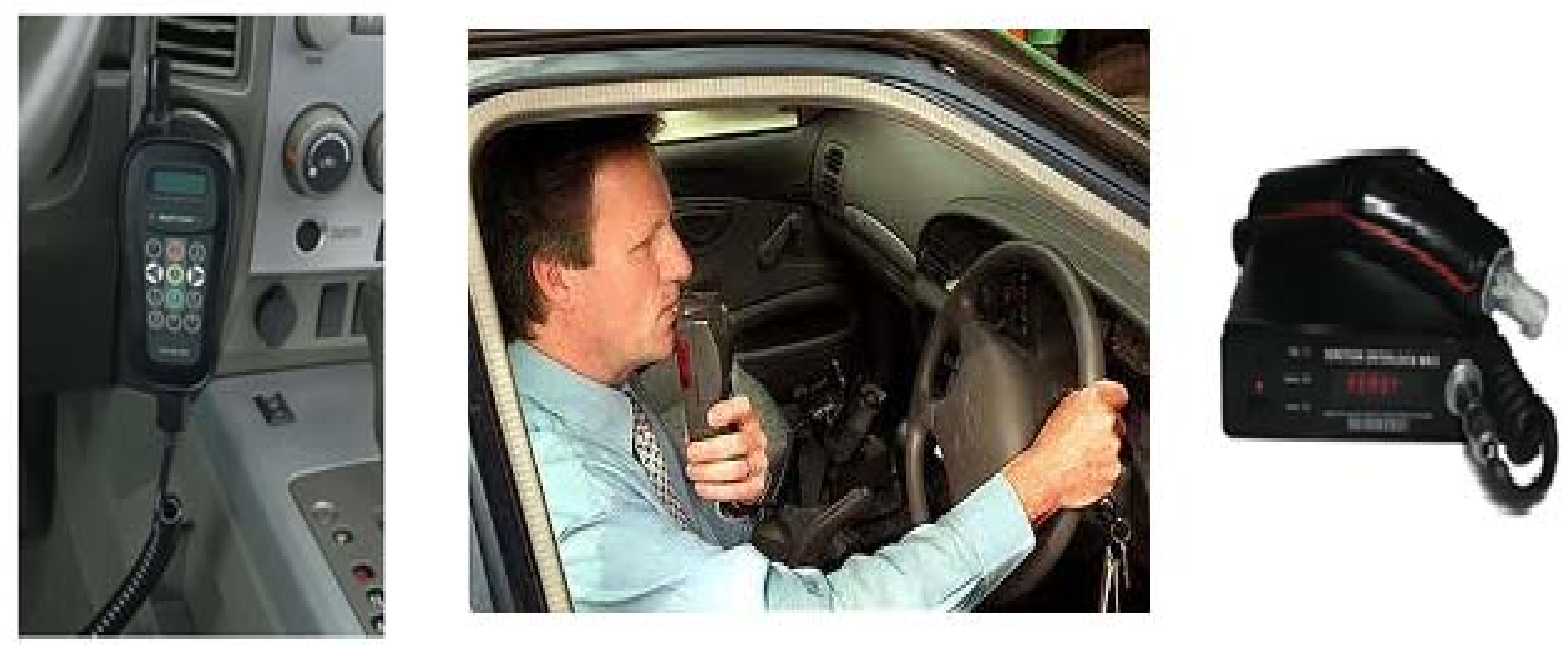

Figure1.1: Available Alcohol Interlock System for Automobile [3] 


\subsection{The current technology and problems for alcohol interlock}

There are three types of sensors available for alcohol interlock systems: semiconductor, fuel cell (electrochemical) and infrared [1]. A semiconductor sensor is cheaper compared to a fuel cell or infrared technology. But it is not ethyl alcohol sensitive and can be triggered by other substances like perfume, cigarette and requires maintenance more often (every 30 days) to maintain the accuracy of the alcohol interlock system. Fuel cell can address the shortcomings of the semiconductor technology as it is ethyl alcohol sensitive, requires less frequent calibration, more reliable and accurate. But it is expensive compared to a semiconductor sensor. Infrared technology is better than semiconductor and it is commonly used for breath testing. However, this technology is impractical for automobile alcohol interlock devices due to its large size. Moreover, this technology is quite expensive and there is a high level of difficulty associated with achieving specificity and accuracy at low BAC levels.

Current technology of alcohol interlock system is based on the electrochemical sensing of breath alcohol. The driver needs to follow an active breath-sampling procedure where the driver has to deeply blow into the sensing unit in order to detect his breath alcohol. This procedure leads to non-continuous monitoring. The system also requires frequent maintenance and is expensive [1].

\subsection{Why FTIRS}

Infrared spectrum represents a fingerprint of a sample with absorption peaks, corresponding to the frequencies of vibrations between the bonds of the atoms that make up the material. No two compounds produce the exact same infrared spectrum as each material is a unique combination of different atoms. Therefore, the quantitative analysis of every different kind of material can be accomplished by infrared spectroscopy. In addition, the size of the peaks in the spectrum is a 
direct indication of the amount of material present. With modern software algorithms, infrared is an excellent tool for quantitative analysis.

Dispersive type infrared spectrometer used individual frequencies from an infrared spectrum using a prism. The working principle of the dispersive type spectrometer is shown in the Fig 1.2. A prism is used to separate the wavelength of the light and a slit is used to restrict the lights that are allowed to strike the detector. The detector measures the amount of energy at each frequency which has passed through the sample. In this type of spectrometer one wavelength is measured at a time results in slow scanning.

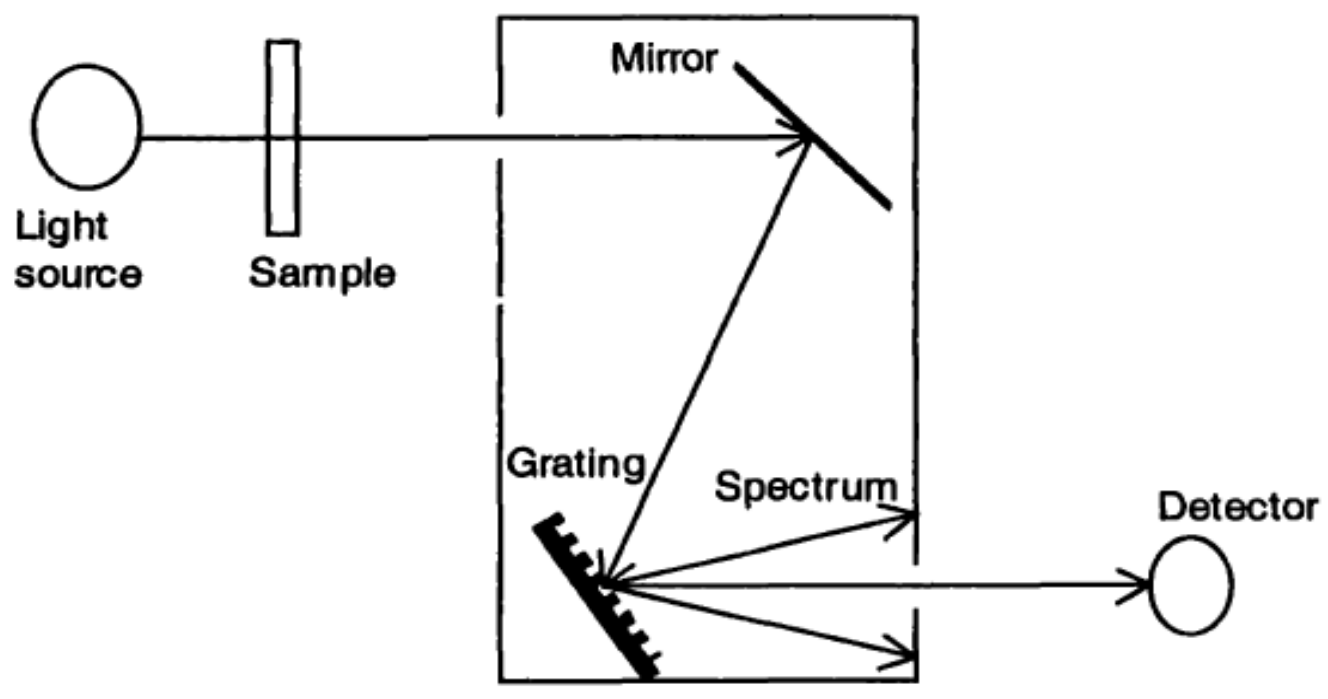

Figure 1.2: Schematic of Dispersive Spectrometer [4]

Fourier Transform Infrared (FTIR) Spectrometer has been developed to overcome the slow scanning limitation of the dispersive spectrometer. It can measure the frequencies simultaneously rather than individually. In order to achieve this criterion an optical instrument called interferometer is used in FTIRS. Interferometer measures the deviations of a surface from a reference plane and creates an interference or fringe pattern and generates a signal consisting of 
all infrared signal encoded in it. The working principle of the Michelson interferometer is shown in Fig 1.3. The incoming light source is divided into two parts at the beam splitter. One beam is transmitted to the fixed mirror and the other beam is transmitted to the moving mirror. These two beams are interfered when they reflected back due to the optical path difference.

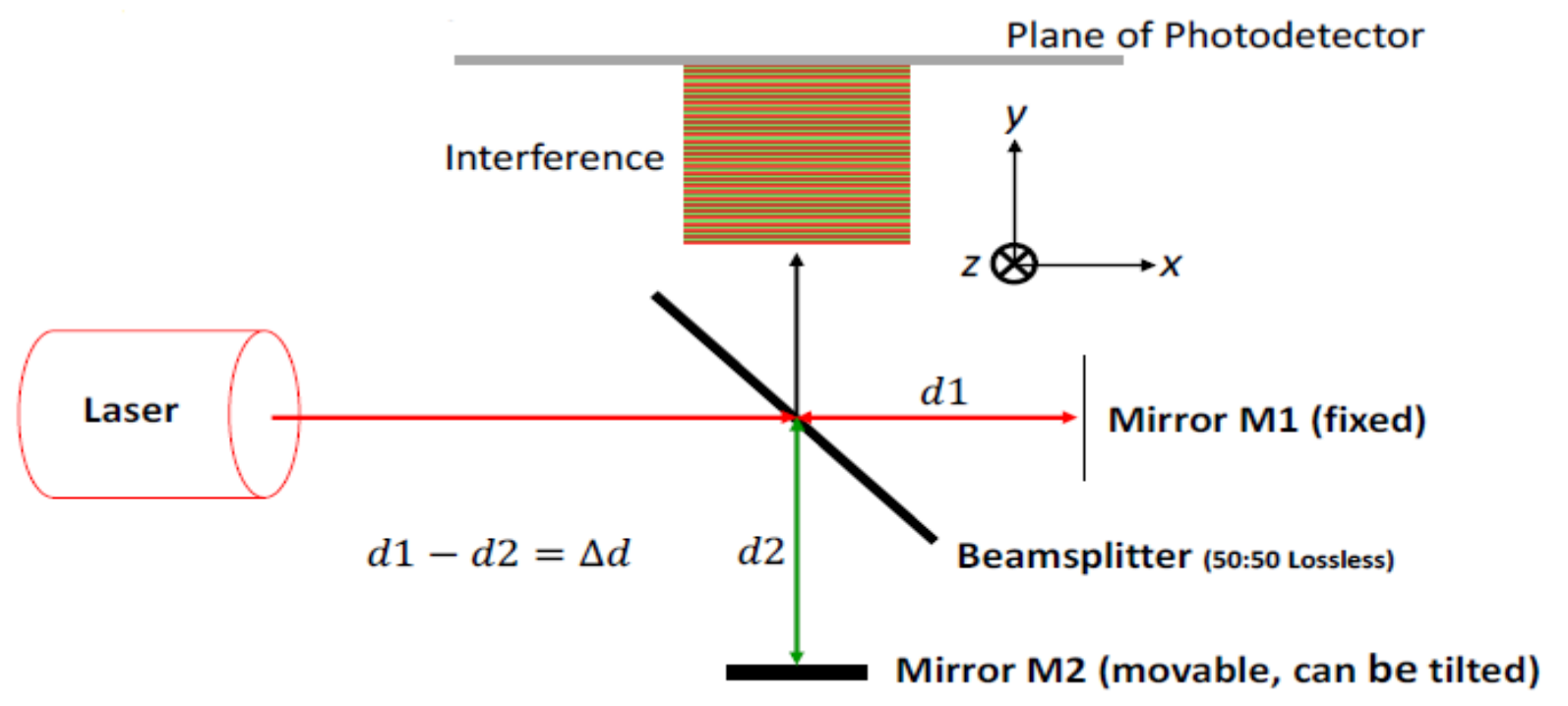

Figure 1.3: Michelson Interferometer [5]

The analysis is performed based on the interference pattern by measuring the number of complete and partial fringes resulting from the translation of the mirror. If moving mirror is not aligned i.e. tilted, the reflected beam will be tilted [6]. As a result, the interference pattern will be distorted. Therefore, it is important to eliminate or minimize the mirror tilting.

The basic layout of a simple FTIRS is shown in Fig 1.4. It consists of interferometer, sample compartment, IR source and detector. The interferogram generated by the interferometer passes through the sample compartment and then creates a fringe or interference pattern on the detector. This procedure can measure all frequencies of the light wave simultaneously which increases the speed of the analysis process. 


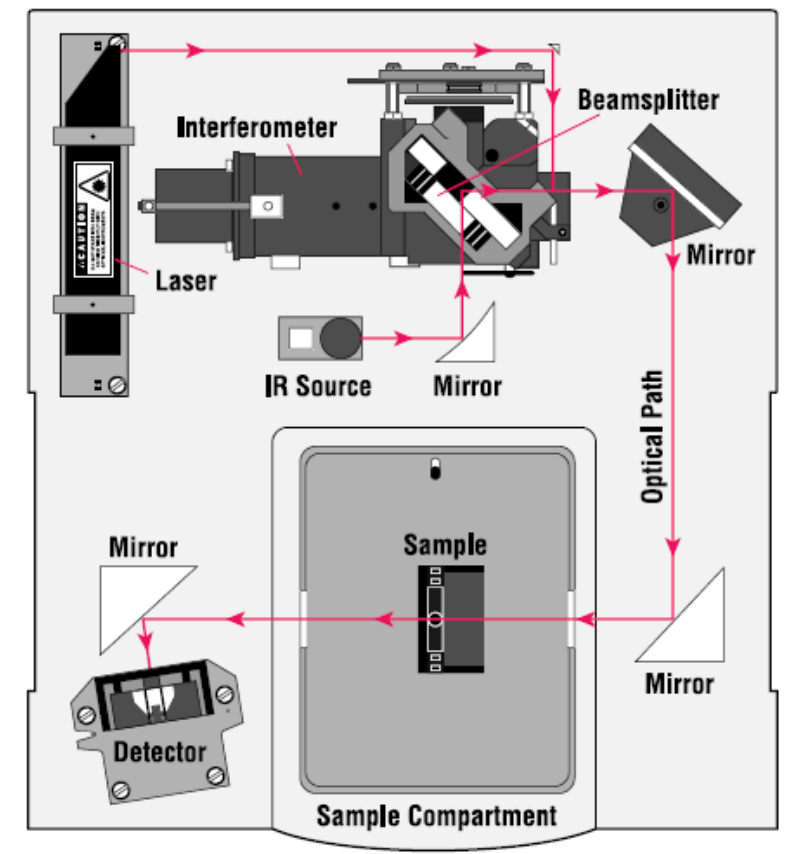

Figure 1.4: A simple FTIRS Layout [9]

\subsection{Why MEMS FTIRS}

Micro Electro Mechanical System (MEMS) is a micro scale structure where mechanical and electrical components integrated on a same chip using the microfabrication process [14]. FTIRS is a well established technique to analyze different solid, liquid and gaseous mixture through creating accurate and high resolution infrared spectra [15][16]. MEMS based FTIRS has the advantage of smaller size, high integration, lower manufacturing cost (for batch production) and high reliability over the regular FTIRS. MEMS based FTIRS also demonstrate the higher sensitivity comparing to the regular FTIR [16].

The current automotive alcohol interlock technology is based on electrochemical sensing of the breath alcohol which has the limitations of low sensitivity. It needs an active breath-sampling procedure which leads to non-continuous monitoring and requires frequent maintenance [1]. 
Alcohol interlock system based on MEMS FTIRS can address the limitations of available alcohol interlock system. MEMS FTIRS based alcohol interlock system would detect breathe alcohol with higher sensitivity than the current technology. So it will ensure continuous monitoring of the driver's BAC without requiring the driver to blow into the sensor. As a result, the effectiveness of the alcohol interlock will be significantly improved. MEMS FTIRS based alcohol interlock system also has the advantage of compact size and low cost.

\subsection{Challenges with MEMS FTIRS}

The main challenge for MEMS FTIRS is to achieve a large displacement in order to get high spectral resolution [16][17][[21][22][31][30]. Spectral resolution is a measure of the ability of an instrument to distinguish spectral features that are close together i.e. it determines the number of data points in a spectrum. For example, a $4 \mathrm{~cm}^{-1}$ resolution spectrum contains a data point every $4 \mathrm{~cm}^{-1}$ whereas a $32 \mathrm{~cm}^{-1}$ spectrum contains a data point at every $32 \mathrm{~cm}^{-1}$.

Another challenge is to maintain alignment precision during mirror actuation [16][21][31]. Optical alignment is important to create accurate interference pattern on the beamsplitter of a Michelson Interferometer.

A large mirror is also required for high sensitivity [17[21][22]. A larger mirror has a larger light gathering power which increases the sensitivity. In 2011, Kenda et al. developed a MEMS actuator for FTIRS and mentioned that the achieving large mirror like $5 \mathrm{~mm}$ is one of the main challenges for MEMS based FTIRS.

A MEMS magnetic actuator has been developed in this thesis to provide the high displacement of the translation micromirror of MEMS FTIRS to overcome the limitation of current alcohol interlock systems. The highest displacement achieved by the existing MEMS FTIRS is 
600micron which used a thermal actuator [21]. The thermal actuator can generate large displacement at static mode. But the actuation substances can be heated up which leads to inaccuracy and less reliability [12]. This heating is the main concern in long term use as polysilicon can deform physically at high temperature. The second highest displacement achieved by existing MEMS FTIRS is 500micron which is achieved in a resonant mode[18]. In resonant actuation mode, the MEMS mirror is actuated with a signal frequency equal to the resonant frequency of the mirror. The mirror motion will act as a mechanical oscillator because the mirror scanning amplitude is amplified at the mechanical resonant frequency and then follow a sinusoidal motion. In static mode, the mirror is actuated far from its mechanical resonant frequency and the actuation signal is linear and the mirror can be driven to hold a specific position by applying a certain continuous actuation signal. In addition, actuator operated at resonant mode requires an expensive vacuum packaging. The highest mirror displacement achieved by the existing MEMS FTIRS at static mode is 54micron[15].

There are many advantages of MEMS magnetic actuator. For example, high actuation force, bidirectional displacement and low voltage requirement. Electromagnetic actuator can generate a large displacement at low voltage even in a static mode [12]. Other advantages of MEMS magnetic actuator are: magnetic actuation eliminates electrical wires which is unavoidable in electrostatic, thermal and piezoelectric actuation. This can drastically reduce the complexity in packaging of MEMS components. Secondly, relatively large magnetic field can be present in free space without causing any damage to environment whereas large electric field in free space or the dielectric create problems such as dielectric breakdown[13]. 


\subsection{Objectives of the thesis}

The main objective of the thesis is as follows:

1. Design a MEMS magnetic actuator capable of a) achieving more than 100 micron static (nonresonant) vertical displacement. This displacement will be highest displacement achieved by available MEMS based FTIRS except the thermal actuator based FTIRS. Thermal actuator is less accurate and not reliable as polysilicon deforms at higher temperature[12]. The highest displacement at static mode achieved so far is 54 micron by the available FTIRS[15]; b) integrating with a micromirror plate to construct the translation micromirror. Two separate microfabrication processes will be used to fabricate the actuator and mirror plate in order to ensure large displacement and high mirror quality; and c) minimizing the tilting.

2. Fabrication of a prototype using mature and commercially available MetalMUMPs process.

One application of the designed MEMS magnetic actuator is in MEMS FTIRS. The actuator will be used to actuate the translation micromirror which is the core part in a Michelson interferometer based FTIRS as shown in Fig 1.5.

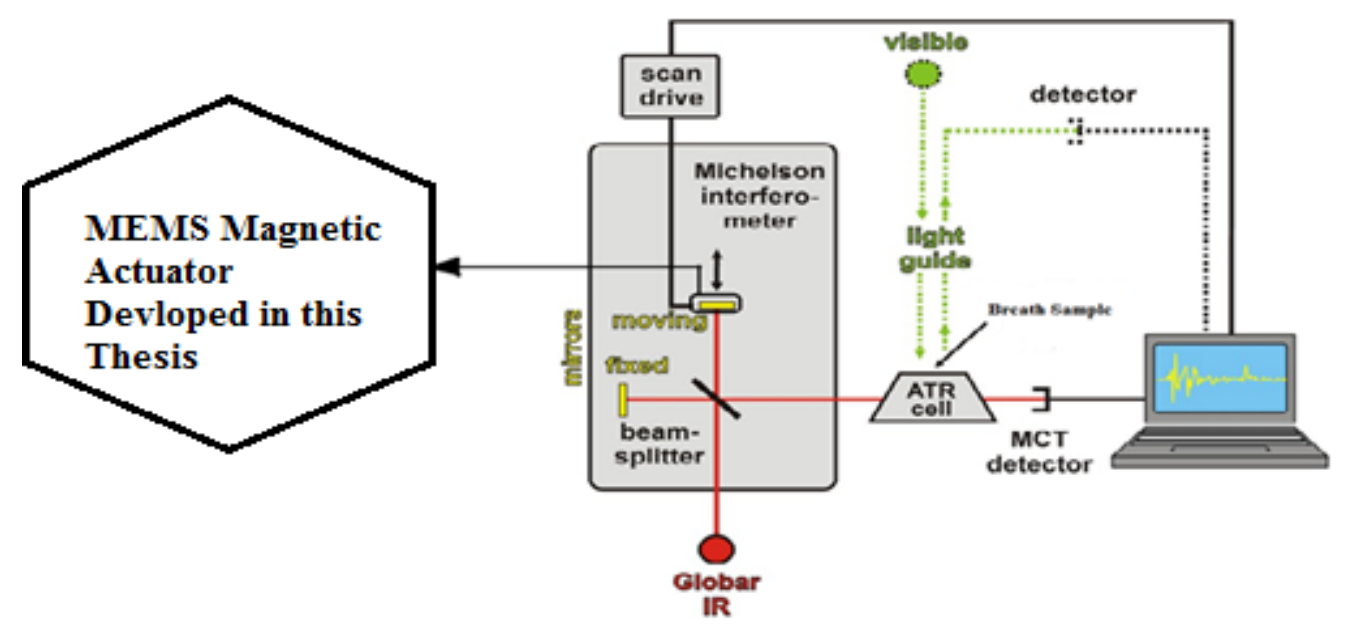

Figure 1.5: Use of Designed Actuator in MEMS FTIRS [7] 


\subsection{Outline of the Thesis}

In Chapter 1 the introduction has been described. In Chapter 2 the background of the FTIRS, MEMS magnetic actuator and their development will be described. Introduction of actuator and realizing translation micromirror using the actuator will be discussed in Chapter 3 . The moving and fixed part design and performances of the actuator will be discussed in Chapter 4 and prototype fabrication will be discussed in Chapter 5. The contributions and future work will be discussed in the concluding chapter which is Chapter 6. 


\section{Chapter 2: Introduction to FTIRS}

FTIRS stands for Fourier Transform Infrared Spectrometer. When infrared radiation interact with the material, it will vibrate the bond of the material and create a molecular fingerprint of the sample [9][10][11][15]. Like human fingerprint, two unique molecular structures cannot produce the same infrared spectrum which makes infrared spectroscopy useful for several types of analysis [9]. FTIR can identify the unknown materials of the sample as well as the amount of the components in a mixture. The one most important advantage of FTIRS technique is that it can measure intensity over a wide range of wavelengths at the same time and this has made the dispersive infrared spectrometer obsolete [9][15]. A Fourier Transform is used to convert the raw data in to the actual spectrum.

\subsection{The Sample Analysis Process}

The sample analysis process using FTIRS is illustrated in Fig 2.1.

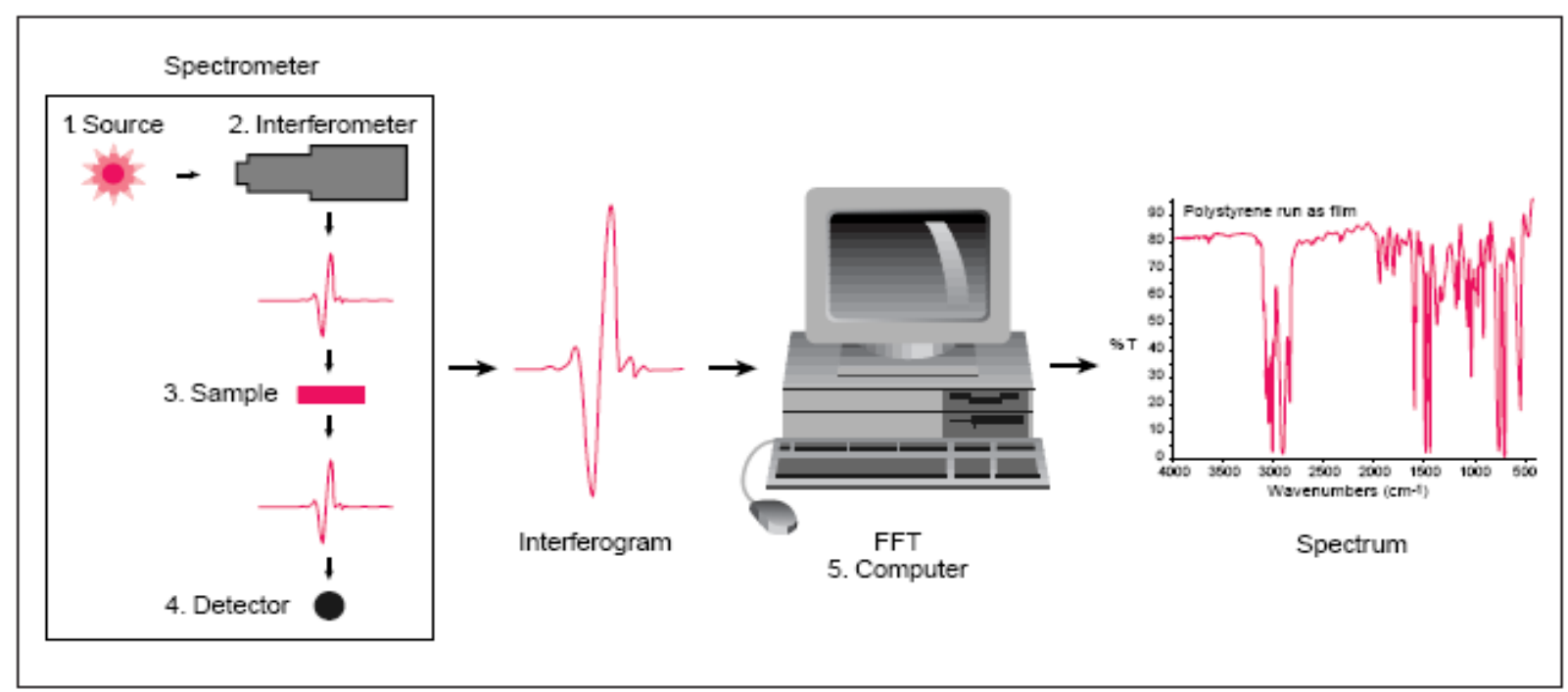

Figure 2.1: Schematic Diagram of Sample Analysis Process using FTIRS [9] 
Infrared energy is emitted from source and the beam divided into two parts when enters into the interferometer. One beam reflects to the moving mirror and another one to the fixed mirror. Since the moving mirror keeps moving at a constant speed and fixed mirror is fixed. There is a phase difference between two beams when they reflected back from both mirrors. The interference is created due to the path difference between two mirrors when they recombined. The interferance signal then exits the interferometer and enters the sample compartment transmitted through or reflected off of the surface of the sample depending on the type of analysis. The specific frequencies of energy for a specific sample are absorbed in the sample compartment for the analysis. The beam finally passes to the detector for final measurement. The detectors used are specially designed to measure the special interferogram signal. The interferogram from the detector cannot be read directly as interferogram plotted the intensity vs. moving mirror position. Therefore, the interferogram is converted to real spectrum i.e. intensity vs. frequency using Fourier Transformation and presented for interpretation and any further manipulation.

The two main advantages which make the FTIRS popular is the Felgett advantage and the Jacquinot advantage [13][15]. Felgett advantage is based on the fact that FTIRS can measure all the frequencies simultaneously and all the wavenumbers of the light beam at the time. The Jacquinot advantage is based on the fact that all the infrared radiation passes through the sample and strikes the detector at once. Thus the detector detects the maximum amount of light at all points during a scan which increases the sensitivity. The other advantages include simple mechanical structure, non-destructive analysis technique and precise measurement method which require no external calibration. But it is impossible to detect atom or monatomic substances, 
noble gases and diatomic molecules such as $\mathrm{N}_{2}$ and $\mathrm{O}_{2}$ by using FTIRS. It is also very difficult to analyze aqueous mixture using infrared as water is a strong infrared absorber.

The application of FTIRS is vast from material identification to quality control and failure analysis. In recent years, it has a huge application on biomedical engineering and space applications.

\subsection{Introduction to MEMS FTIRS}

MEMS based FTIRS has been developed for various applications by different groups [16-32] due to its benefit over regular FTIRS. All these groups have described that the MEMS based FTIRS has the advantages of high spectral resolution which increase the sensitivity of the spectrometer. The miniature size of the MEMS based FTIRS has made it popular for biomedical, space, environmental and other application. The manufacturing cost of the MEMS FTIRS is also low due to the leveraging of microfabrication batch production [17] [21] [22].

\subsubsection{Different Design Approach of MEMS based FTIRS:}

There are three different design approaches for MEMS based FTIRS. They are Michelson Interferometer based FTIRS, Lamellar Grating Interferometer (LGI) FTIRS and standing wave Interferometer based FTIRS.

The most common and widely used MEMS FTIRS is Michelson Interferometer based FTITS. This type of FTIRS has higher sensitivity and can maintain precise optical alignment $[16][17][20][21][22][23]$. The basic design included a classical Michelson interferometer with a MEMS actuator to displace the moving mirror as shown in the Fig 2.2. Michelson interferometer based MEMS FTIRS become popular due to its simple operation technique and stability [16]. 


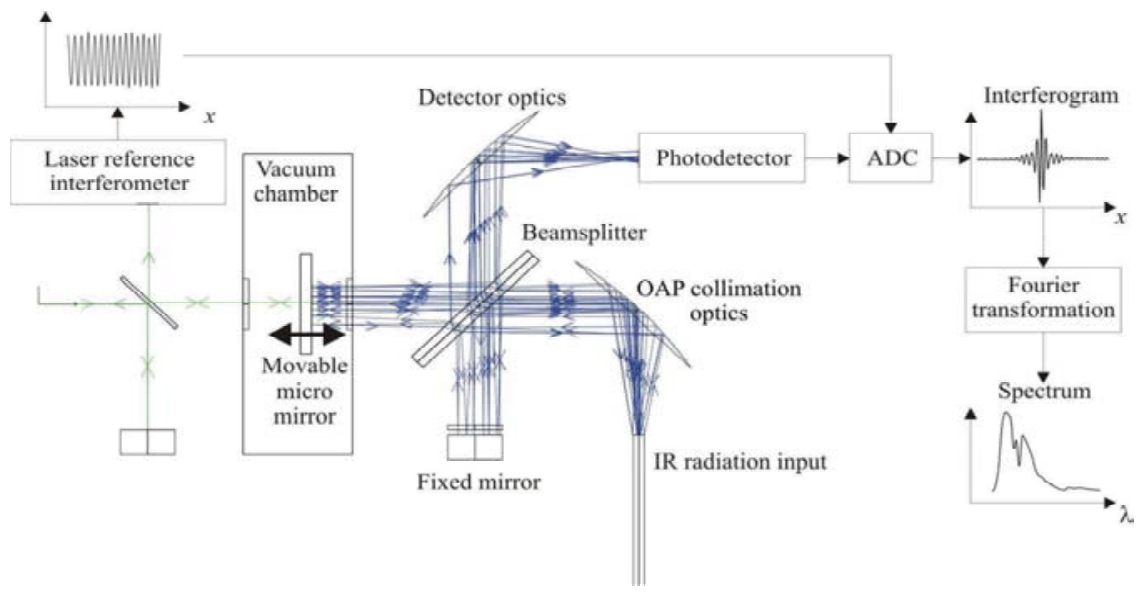

Figure 2.2: Layout of Michelson Interferometer based FTIRS [17]

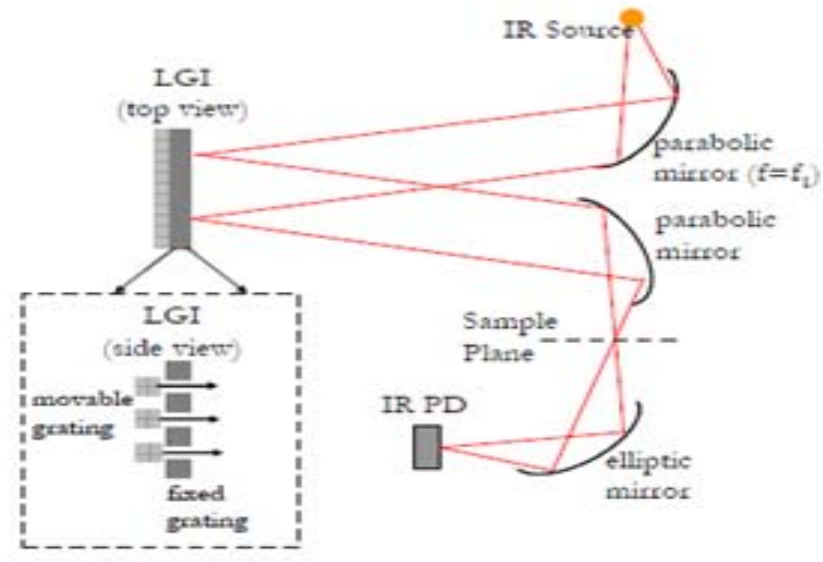

Figure 2.3: LGI based FTIRS [18]

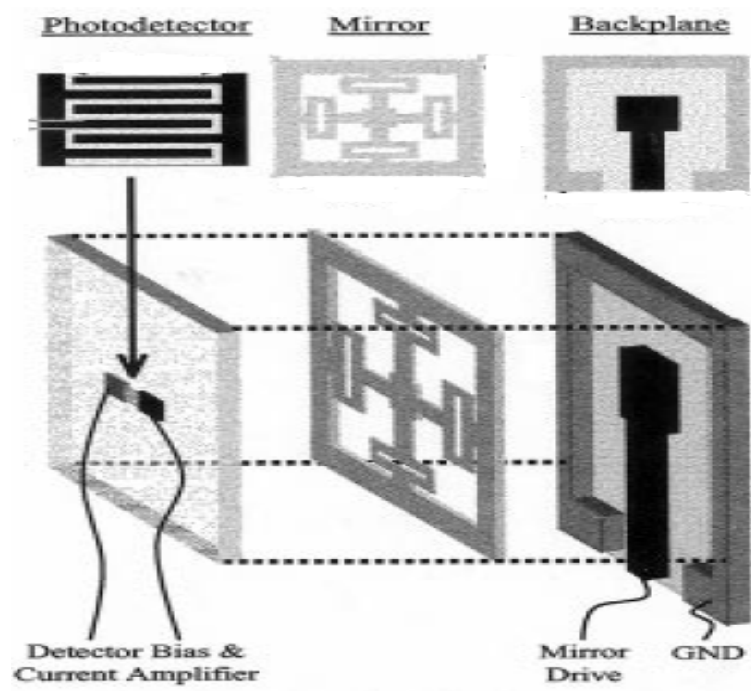

Figure 2.4: Standing Wave based FTIRS [29] 
Lamellar Grating Interferometer has become popular after the advancement of microfabrication. MEMS technology can facilitate the fabrication of very precise diffraction grating [18] [24] [19] [26]. Working principle of LGI based FTIRS is shown in Fig 2.3. The incoming beam is reflected partly from the fixed grating fingers and partly from the moving grating fingers. When these two beams are interfered with each other, they create interferogram at the detector due to the optical path difference. In order to analyze a sample, the sample needs to be placed in the sample plane and the transmittance properties of the sample can be detected by comparing with the source spectrum without the sample [18]. The main advantages of lamellar grating FTIRS are the elimination of beam splitter and reference mirror which make the interferometer compact and less expensive. But the complex fabrication process is one of the main disadvantages of this type of MEMS FTIRS.

The interferogram generated by the superposition of forward and backward propagating waves is known as a standing wave [20] as shown in Fig 2.5. This type of FTIRS also has no beam splitter which reduces the larger physical size.

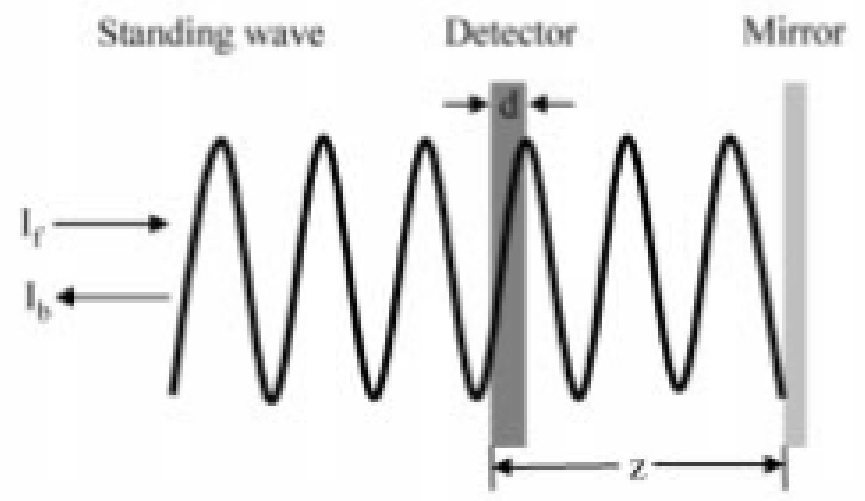

Figure 2.5: Schematic of a Standing-wave [29]

The standing wave spectrometer consists of a MEMS mirror, a thin-film semitransparent photo detector and an electrical back plane for actuating the mirror as shown in Fig 2.4. The fixed detector is used to sample the standing wave and the moving mirror is used to vary the standing 
wave [20] [28] [29]. When light is reflected from a mirror, the standing wave pattern is generated because of the interference of the backward propagating and forward propagating waves. The forward propagating wave represents the fixed arm whereas the backward propagating wave represents the moving arm of a Michelson Interferometer.

\subsubsection{MEMS FTIRS with High Sensitivity}

The main goal of the thesis is to design a MEMS magnetic actuator for a MEMS FTIRS based alcohol interlock system. This will overcome the limitations of available alcohol interlock systems e.g. driver does not need to breath into the machine when detecting the alcohol level due to the high sensitivity. High spectral resolution can be achieved by larger translation of the moving mirror. Block MEMS, LLC developed a MEMS based FTIR fabricated by SUMMiT V fabrication process developed by Sandia National Laboratory [21] which can provide $600 \mu \mathrm{m}$ physical mirror displacement (spectral resolution of $8 \mathrm{~cm}^{-1}$ ). A combination of thermal actuator with a gear and crank system is used in a Michelson Interferometer setup as shown in Fig 2.6. The basic design includes stationary mirror, moving mirror and beamsplitter that are constructed in the same substrate and in the same surface micromachining fabrication process. These three

components are identical in their construction. The beamsplitter is elongated by $\sqrt{ } 2$ in order to achieve a $45^{\circ}$ alignment. Each component has a primary component with an optical surface of interest, a secondary component which holds the primary component in place to maintain the interferometric alignment and a proprietary self-locking mechanism to enable irreversible assembly. 


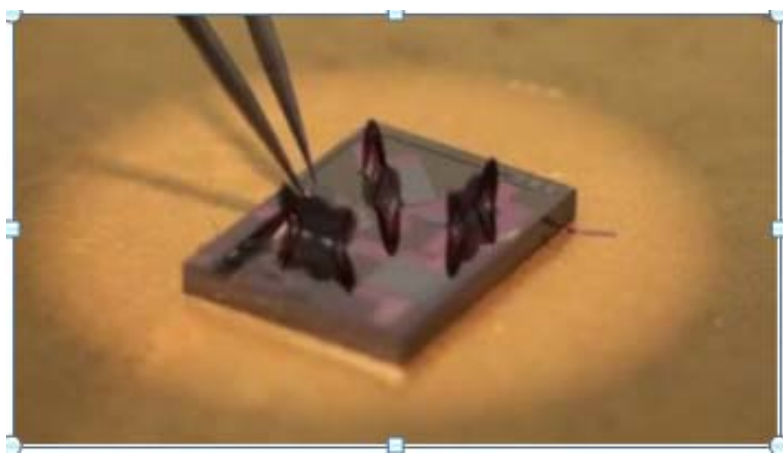

(a)

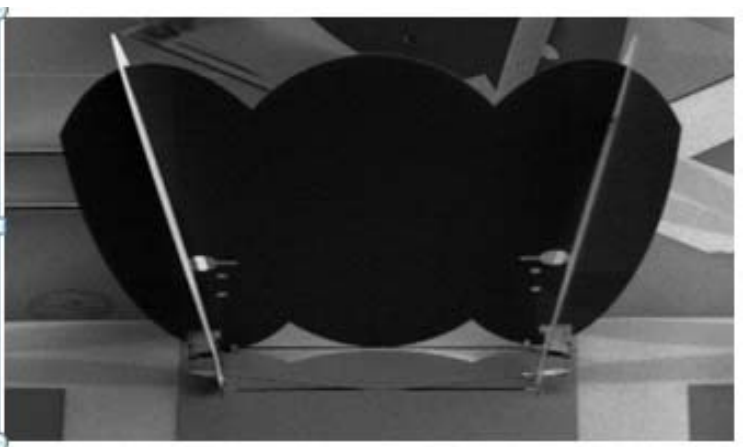

(b)

Figure 2.6: (a) MEMS Michelson Interferometer by Block MEMS, (b) SEM Micrograph [21]

Chempen design can provide the maximum mirror size, higher flatness and less tilt compared to MEMS component. It is capable of producing a $600 \mu \mathrm{m}$ mirror displacement which is a large mirror travel for MEMS FTIRS [21].

Fraunhofer Institute for Photonic Microsystems (IPMS) designed a MOEMS mirror to enable a miniaturized MEMS based FTIR with improved system performances of $10 \mathrm{~cm}-1$ spectral resolution and has a target of achieving $500 \mu \mathrm{m}$ mirror translation at resonant mode [18]. The moving mirror is translated by four symmetric pantographs like suspension as shown in Fig 2.7.

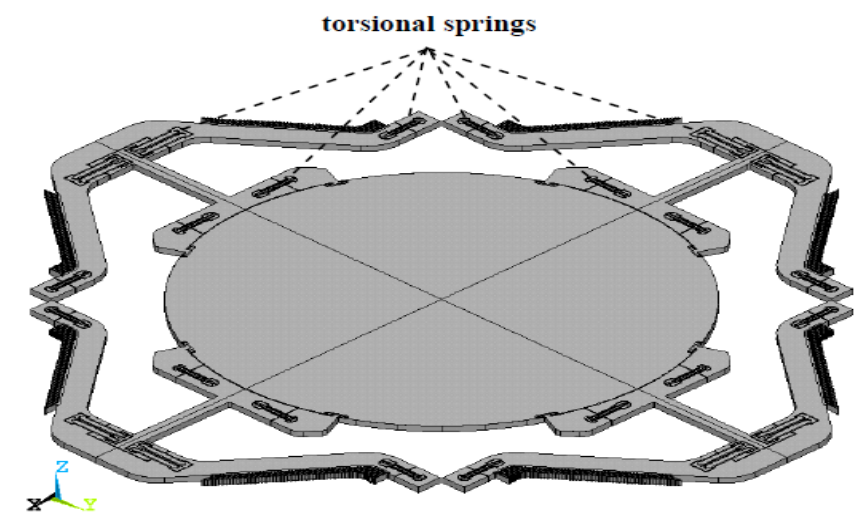

Figure 2.7: Schematic Design of the Mirror Plate and Basic Pantograph Suspension Designed by IPMS [18] 
Torsional type springs are used as deflectable element instead of bending spring in order to reduce mirror deformation due to the mechanical stress. One single pantograph consists of six torsional springs and connected by stiff levers.

Guckelet al. used LIGA (German abbreviation and stands for Lithographie, Galvanoformung and Abformung) technology to fabricate a MEMS FTIRS [15]. It is basically a Michelson Interferometer which consists of an optical bench for the passive alignment of the optical components with an integrated actuator shown in Fig 2.8. All components were fabricated in the same plane using LIGA technology. An electromagnetic actuator is used to provide the large displacement. This type of spectrometer is capable of achieving optical path difference of 108 $\mu \mathrm{m}$ corresponding to mirror travel of $54 \mu \mathrm{m}$.

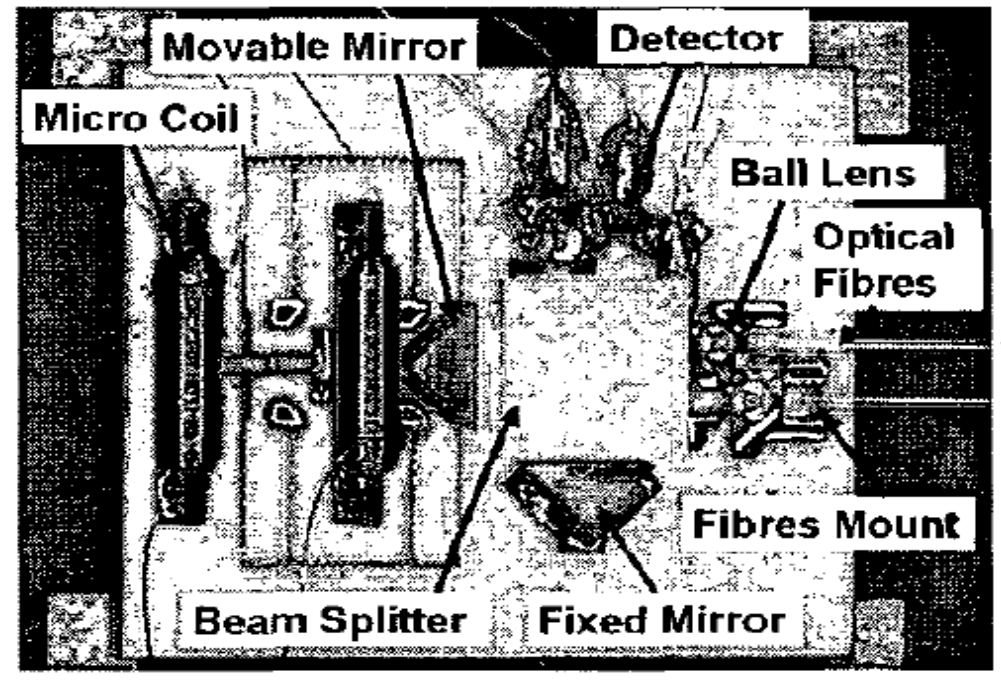

Figure 2.8: Top View of the System [15]

In 2009, Feiweenet al. designed a FTIR actuated electromagnetically at resonant mode which achieved 100 micron displacement [19]. Omar et al. designed an FTIR in 2000 capable of achieving 39 micron displacement at static mode [27]. The summary of existing MEMS FTIRS is shown in Table 2.1: 
Table 2.1: Existing MEMS FTIRS and their Actuators

\begin{tabular}{|l|l|l|l|l|}
\hline & $\begin{array}{l}\text { Interferometer } \\
\text { Type }\end{array}$ & Actuator Type & $\begin{array}{l}\text { Mode of } \\
\text { Operation }\end{array}$ & $\begin{array}{l}\text { Displacemen } \\
\text { tmicron) }\end{array}$ \\
\hline Chempen & $\begin{array}{l}\text { Michelson } \\
\text { Interferometer }\end{array}$ & Thermal & 600 \\
\hline IPMS & Michelson & $\begin{array}{l}\text { Electrostatic } \\
\text { Vertical comb } \\
\text { drive }\end{array}$ & Resonant & 500 \\
\hline $\begin{array}{l}\text { Feiweenet } \\
\text { al. }\end{array}$ & Lamellar Grating & Comb drive & Resonant & 100 \\
\hline Guckelet al & Michelson & Magnetic & Static & 54 \\
\hline Omar et al. & Michelson & $\begin{array}{l}\text { Electrostatic Comb } \\
\text { drive }\end{array}$ & Static & 39 \\
\hline
\end{tabular}

\subsection{MEMS magnetic actuators}

The main challenge of developing MEMS FTIRS is to provide large displacement of the moving mirror at static mode. This challenge can be overcome by developing a MEMS magnetic actuator. MEMS magnetic actuators have the advantage of achieving large displacement at low voltage in static mode. One of the main objectives of this thesis was to design and fabricate a magnetic actuator providing large displacement which would be integrated in the MEMS FTIRS to facilitate the translation of the moving mirror. The description of available MEMS magnetic actuators are as follows:

Magnetic Design 1[23]: In 2002, Chao and Ahn designed a bidirectional magnetic actuator which can provide a deflection of $+/-80$ micron deflection approximately when applying a dc current of $100 \mathrm{~mA}$. As shown in Fig 2.9, the actuator consists of a silicon cantilever beam and an electromagnet. There are arrays of permanent magnet at the tip of the beam to provide the bidirectional motion. At the bottom surface of the beam the arrays permanent magnet are placed along the axis of the electromagnet. 


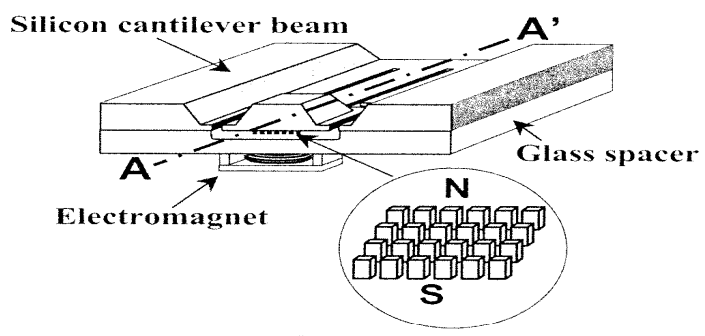

Permanent magnet arrays

(a)

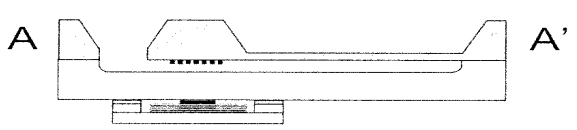

(b)

Figure 2.9: Structure of a cantilever beam microactuator: (a) schematic view and (b) cut view [23] Magnetic Design 2: Cho et al. designed an electromagnetically driven micromirror which is capable of inducing both $\mathrm{x}$ and $\mathrm{y}$ axis actuation and used an external magnetic field to acquire large force. The actuation voltages are below $4.7 \mathrm{~V}$ and $1.76 \mathrm{~V}$ for $\mathrm{x}$-axis and $\mathrm{y}$-axis actuation respectively.

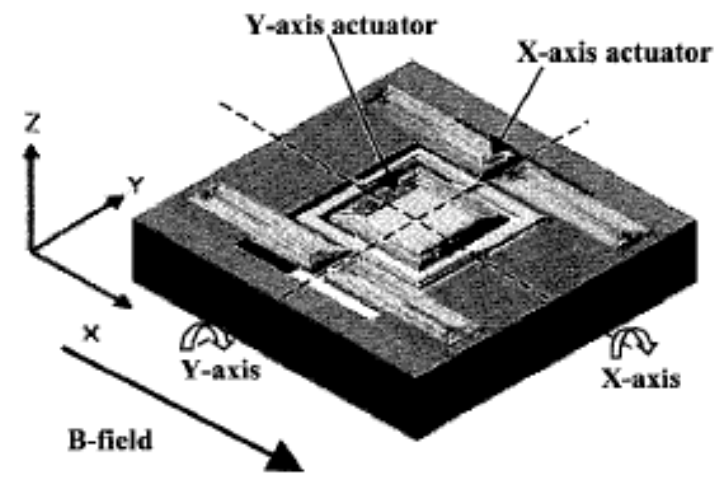

Figure 2.10: Schematic Diagram of Two-axis Electromagnetically Actuated Micromirror [21]

As shown in the Fig 2.10, the structure consists of a mirror plate, frames, cantilever type actuator and coils. There are two types of actuator. The $\mathrm{x}$-axis actuator is in the cantilever on the outside of the mirror plate and the y-axis actuator is in the mirror plate. The operating direction of the 
micromirror is determined by the direction of the current flowing in the coils on the mirror plate and the cantilever actuator.

Magnetic Design3: In 2008, Lee et al. [24] designed an electromagnetic actuator capable of achieving a deflection of 150 micron. This electromagnetic actuator consists of a diaphragm, a micro coil, and a magnet shown in Fig 2.11. The actuation occurs as the magnetic force due the applied electrical current between the micro coils caused the diaphragm to deflect. As shown in the figure below the micro actuator consists of micro coil electroplated onto the glass substrate and the PDMS diaphragm.

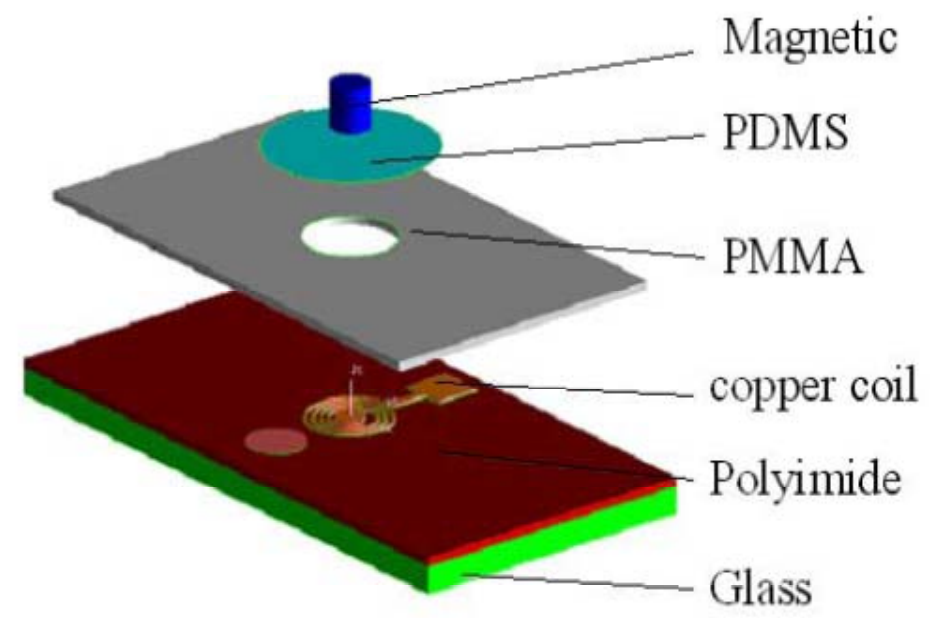

Figure 2.11: Sketch for the Construction of the Fabricated Electromagnetic Actuator [24]

Magnetic Design 4: In 2009, Cho et al. designed an electromagnetic actuator which is capable of $\mathrm{x}, \mathrm{y}$ and $\mathrm{z}$ direction actuation. The maximum vertical displacement achieved +-42 micrometer and the actuation voltage is less than $3 \mathrm{~V}$. It is fabricated by bulk micromachining process. The $\mathrm{z}-$ axis actuation can provide the easy assembling of optical components as well as improving the alignment of the components. 


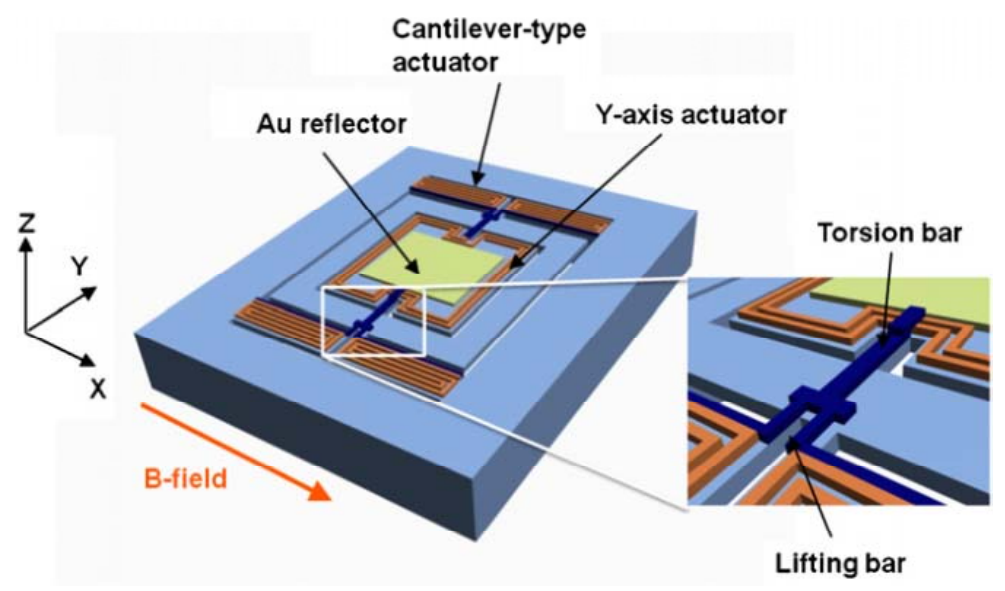

Figure 2.12: Schematic Diagram of Three-axis Electromagnetically Actuated Micromirror [22]

As shown in Fig 2.12, the structure consists of a gold coated mirror plate, coil, frame and cantilever type actuator. The actuator coil in the mirror plate can provide y-axis actuation whereas the cantilever type actuator provide both $\mathrm{x}$ and $\mathrm{z}$ axis actuation.

Magnetic Design 5[16]: Guckel et al. designed an actuator where the magnetic coil is externally fabricated and assembled along the magnetic path in order to avoid the complex fabrication technique and increase the number of turns of the coil. Moreover the increased inductance of these coils improves their suitability for inductive position sensing. The actuator has three parts: the coil, the substrate and the spring constrained plunger. The coil converts the electric energy to magnetic energy, the substrate acts as a mount for the coil and springs, and has a magnetic path for coupling the magnetic field from the coil to the plunger. It is fabricated using LIGA technology.

Magnetic Design 6[28]: Liu et al. designed a three dimensional structure for micro electromagnetic actuator. The three-dimensional structure and customized fabrication process is adopted to improve the magnetic force and reduce the rigidity of the Permalloy cantilever. The design of the electromagnetic actuator mainly consists of three components: a planar exciting coil, a Permalloy cantilever beam and a lower Permalloy flux return path as shown in Fig 2.13. 
The planar exciting coil will generate a magnetic flux when a current pass through. Most of the flux concentrates in the Permalloy magnetic core. The Permalloy cantilever bend down due to the attraction to the magnetic force.

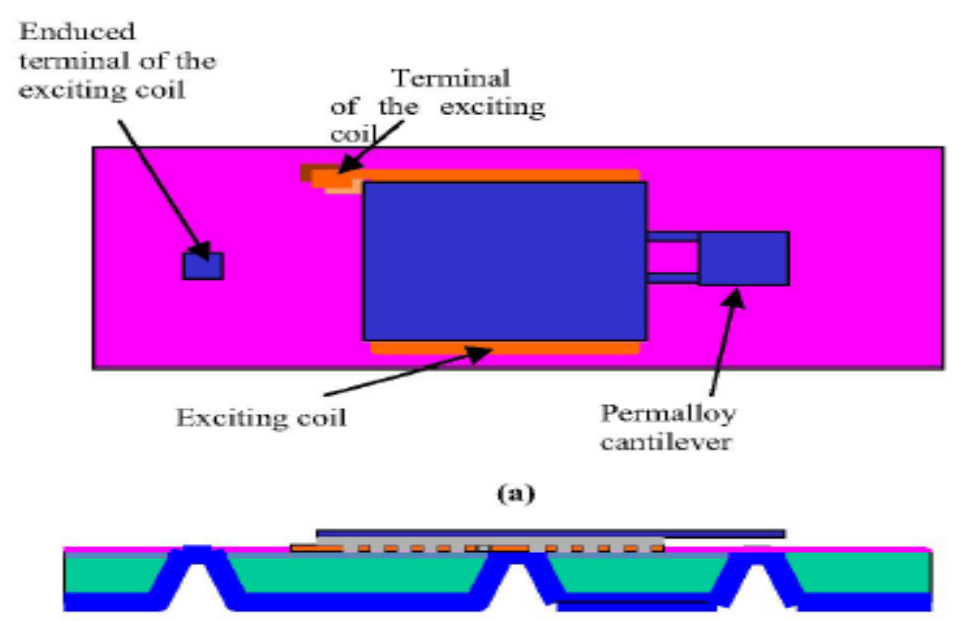

(b)

Figure 2.13: Schematic of the Electromagnetic Actuator. (a) Overhead View of the Micro Actuator. (b) Side View of the Micro Actuator [28]

Magnetic Design 7 [25]: In 2011, Kim et al. introduced a MEMS antenna platform working at $60 \mathrm{GHz}$ resonant frequency where they used millimeter scale electromagnetic actuator. The magnetic force is generated by four electromagnets composed of copper coil wound around iron cores. The structure of the actuator is shown in Fig 2.14

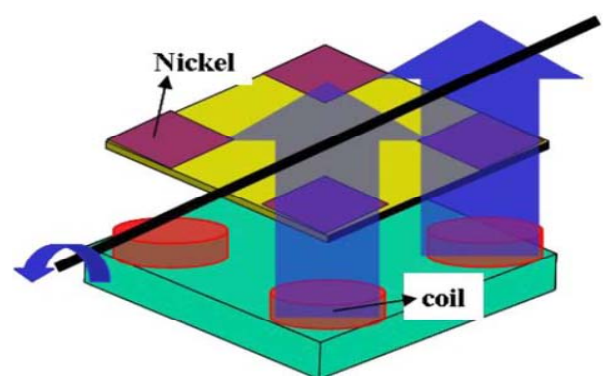

Figure 2.14: MEMS Antenna Driven by Electromagnetic Actuator [25]

A maximum displacement of $1.3 \mathrm{~mm}$ is obtained at $59.8 \mathrm{GHz}$ resonant frequency and the structure is fabricated using a complicated customized MEMS fabrication technology. 


\section{Chapter 3: Actuator and Translation Micromirror}

In this thesis a MEMS magnetic actuator has been designed and fabricated using METALMUMPS process. The goal of the thesis is to develop a high displacement magnetic actuator with potential application to MEMS FTIRS with increased sensitivity. This MEMS FTIRS will be used as future alcohol interlock system which can provide continuous operation without requiring the driver to breath into the device. In order to achieve high sensitivity and higher spectral resolution, it is required to be detected by a large mirror displacement. MEMS magnetic actuator can play an important role in providing a large displacement. Commercial METALMUMPS process for fabrication has the advantages of providing reliable and low cost fabrication for MEMS component. In this Chapter all components of MEMS magnetic actuator will be discussed

\subsection{Magnetic driven micromirror for FTIRS:}

There are three types design approach in MEMS FTIRS: Michelson interferometer based FTIRS, LGI based FTIRS and standing wave based FTIRS as discussed in Chapter 2. For the advantages over other design approaches, Michelson interferometer based FTIRS design approach will be used to develop MEMS based FTIRS for the future breath alcohol detector.

In the Michelson interferometer, there is a translation mirror and a fixed mirror. The actuation mechanism for the displacement of the moving mirror is very crucial in this application. The spectral resolution of FTIR is proportional to the displacement of the translation micromirror. There are different actuation methods in the field of MEMS application. They are electrostatic, thermal, magnetic and piezoelectric actuation. The electrostatic actuator can induce the large displacement but it requires high voltage (more than 100V) to generate the large displacement. 
Moreover the electrostatic actuator needs to be at the resonant mode in order to generate the large deflection. The thermal actuator can generate large displacement but there is a possibility of heating and is not feasible in case of long runs as polysilicon deform at high temperature. But the electromagnetic actuator can generate large displacement at low voltage even in a static mode.

MEMS magnetic actuator has been designed and fabricated to provide the displacement of the translation mirror. The micromirror will be fabricated and bonded on this actuator plate to construct a translation micromirror. The approximate arrangement of the translation micromirror is shown in Fig 3.1.

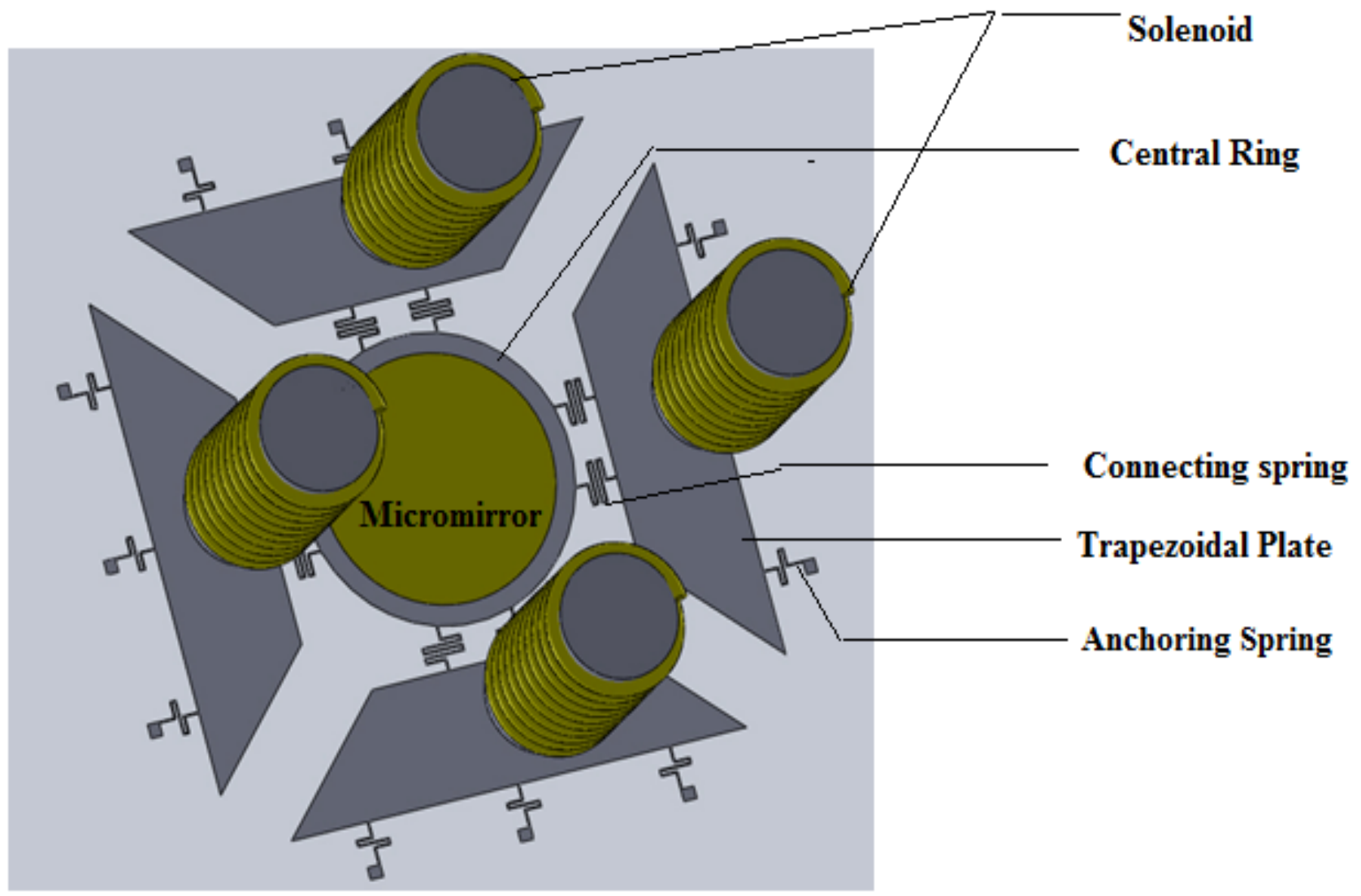

Figure 3.1: Magnetic Driven Micromirror Assembly 
The magnetic driven micromirror assembly consists of an actuator and a micromirror. The actuator has two parts: moving part and fixed part. The moving part consists of four trapezoidal plates which are symmetrical to each other and are connected to a central ring. The trapezoidal plates are fabricated with electroplated nickel (Permalloy material). There are three anchoring springs at the larger side of each the trapezoidal plates which are fixed at the end. There are two connecting springs at the shorter side of the each trapezoidal part which are connected to the central ring. The moving part is fabricated using commercial and mature MetalMUMPs process. The micromirror will be fabricated separately and will be glued with the central ring of the moving part of the actuator after fabrication. The fixed part of the actuator consists of four solenoids placed above the four trapezoidal plates. Trapezoidal plates will rotate with the interaction of the magnetic field generated by each solenoid. The rotations of the trapezoidal plates provide translation to the central ring.

\subsection{Working principle of the magnetic actuator}

The actuator designed in this thesis converts rotational motion of the four trapezoidal plates into translational motion to the central ring of the micromirror due to the interaction between magnetic field and Permalloy material nickel.

MEMS magnetic actuators use magnetic effects to produce motion on the moving part of the microstructure. This magnetic effect can be created by the permanent magnet and /or electromagnetic coil. Magnetic force can be produced either of the two ways:

i. Current carrying conductor exposed to a magnetic field is known as Lorentz Force

ii. Force generated by varying the reluctivity of the magnetic coil along its movement path is known as Reluctance Force 
The either one of the above two forces or both forces together are used to design the magnetic actuation.

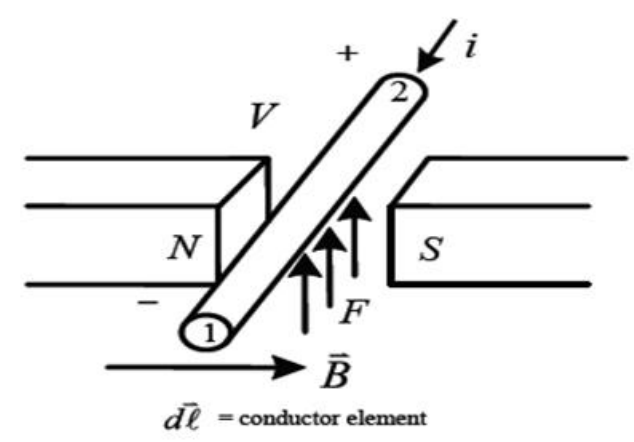

Figure 3.2: Magnetic Actuation [13]

Magnetic field density B represents the induced total magnetic field inside a piece of magnetic material as shown in Fig 3.2.

Uniform and non-uniform magnetic fields are two broad categories of magnetic field irrespective of the methods by which they are generated. The interaction of hard and soft magnet in uniform and non-uniform magnetic fields has been illustrated in the Fig 3.3.

Net force and/or torque that can be produced depend on the type of magnet (hard or soft) and their initial orientation in the field (aligned or misaligned with field lines). Two hard (permanent) magnets (1and 2) and two soft (Permalloy) are used to illustrate the magnetic actuation with different orientation. Pieces 1 and 3 are aligned to the magnetic field whereas the pieces 2 and 4 are misaligned with the magnetic field.

When there is no external magnetic field as shown in Fig 3.3 (a), the permanent magnets are polarized but the soft magnets are not polarized. The force and torque is not present on any of the pieces due to the absence of an external magnetic field. 


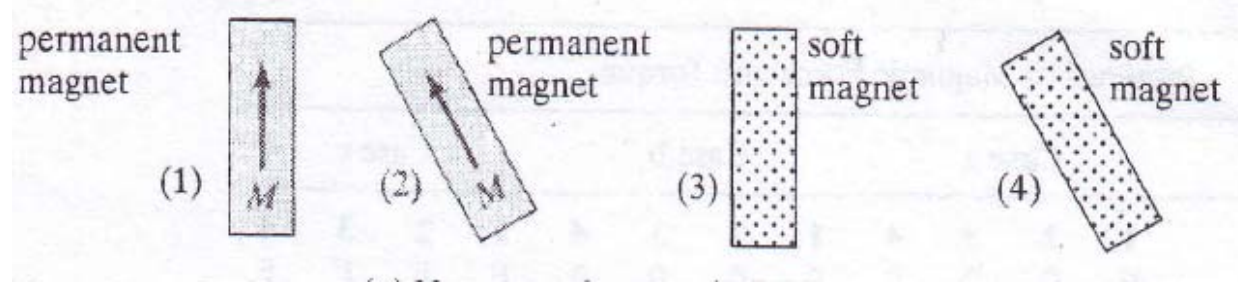

(a) No external magnetic field

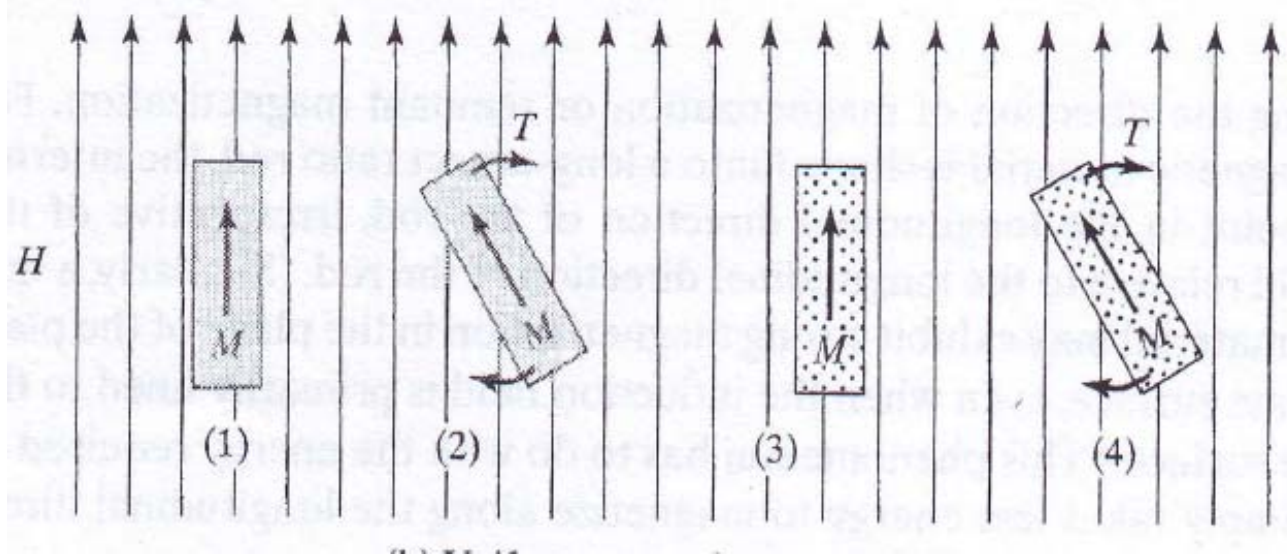

(b) Uniform external magnetic field

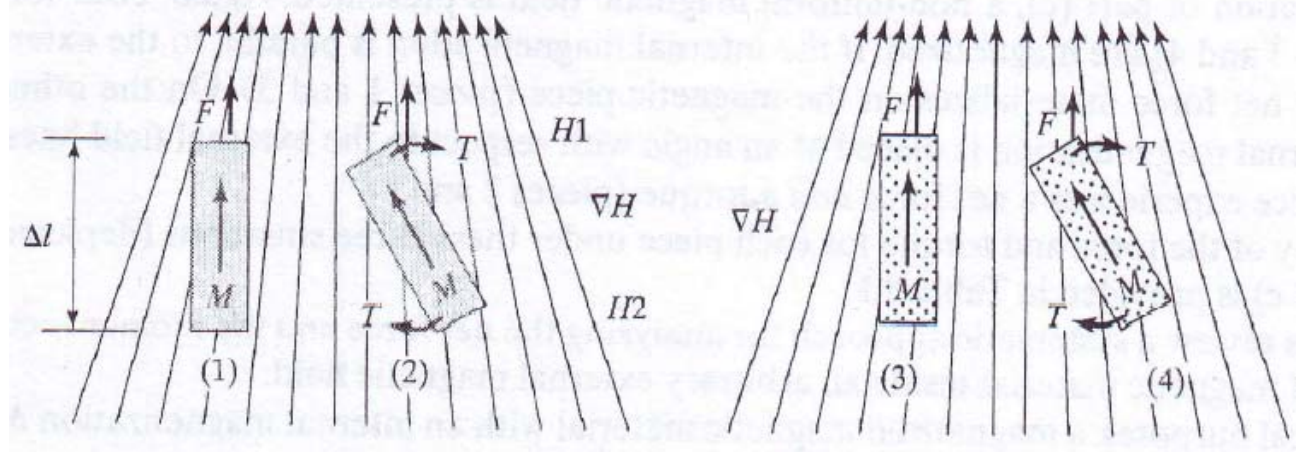

(c) Non-uniform external magnetic field

Figure 3.3: Magnetization and Force States in Magnetic Field [13]

When a uniform magnetic field is applied as illustrated in Fig 3.3(b), both the hard and soft magnets become magnetized. No force or torque is generated on the aligned pieces (1 and 3) parallel to the magnetic field. But a torque is generated to the misaligned pieces ( 2 and 4$)$ as the internal magnetization being placed at an angle with respect to the external magnetic field. Shape 
anisotropy plays an important role to determination the direction of actuation due to this magnetization effect.

In Fig 3.3(c), when non-uniform magnetic field is applied, both the hard and soft magnets become magnetized. A net force is generated on the aligned pieces (1 and 3) whose internal magnetization is parallel to the external magnetic field. On the other hand, a net force and torque are generated on the misaligned pieces (2 and 4) whose internal magnetization is placed at an angle with respect to the external magnetic field.

The top surface of the trapezoidal plate has been electroplated with nickel (Permalloy/soft magnet) as shown in Fig 3.1. Therefore, this trapezoidal plate generates force and torque at the presence of external magnetic field. When an external magnetic field is applied through the solenoid, an internal magnetization is created within the soft magnet or Permalloy pieces which subsequently interact with the external magnetic field. This will create a magnetic torque causing the trapezoidal plates to rotate. The deflection of a mechanical structure for actuation due to the magnetic field depends on different factors and parameters of the device. There has to be an applied force and a restoring force during actuation. The applied force is the force represented by magnetic interaction and the restoring force is determined by the spring constant of the moving structure. The applied force depends on both the field from the coils and the magnet. The applied force depends on the remanence value of the magnet and its volume and the amount of current passing through it. The restoring force depends on the spring constant, Young's modulus of the moving structure, its length, width and thickness. The working principle of the designed magnetic actuator in this thesis can be illustrated by the Fig 3.4. 


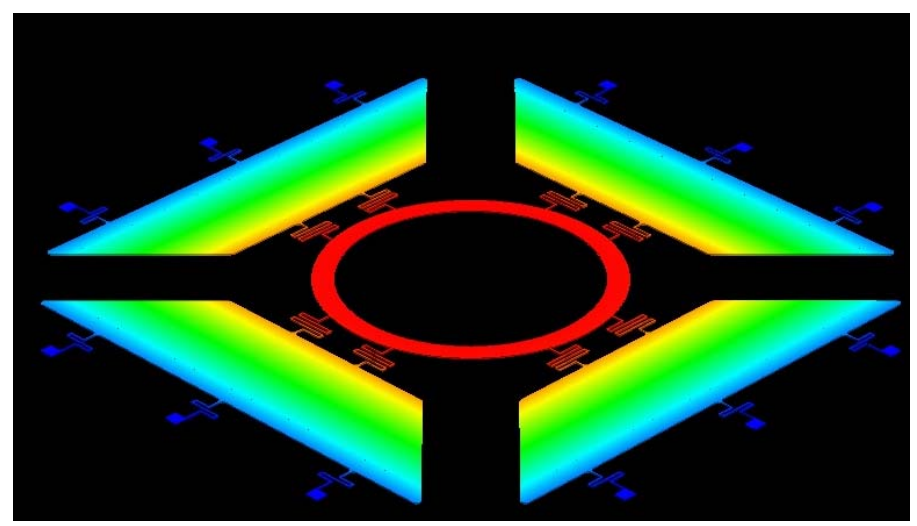

(a)

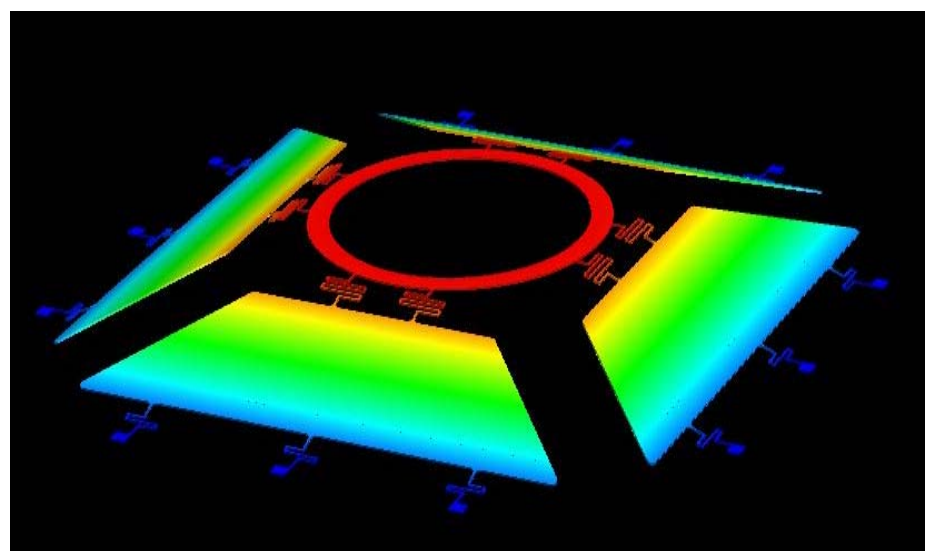

(b)

Figure 3.4: Illustration of Actuation Principle. (a) Rest Position, (b) Out of Plane Actuation due to External Magnetic Field (Fixed part is not shown in the figure)

The structure of the MEMS magnetic actuator has been discussed in Chapter 3.1, where there are four solenoids placed above each trapezoidal plate of the actuator. A magnetic field is generated by the solenoid when current is applied. The torque results due to the interaction between the magnetic field and the Permalloy trapezoidal plate. This torque will rotate the trapezoidal plates and will raise the central ring through the connection springs and anchoring springs. Thus the micromirror assembled in the central ring will also rise. When the current through the four solenoids are removed, the trapezoidal plates and the ring along with the micromirror plate will go back to its original positions due to the restoring forces from the springs and hence the micromirror will return to its original position. Mirror tilting will be minimized in this design by 
determining the current requirement of each solenoid to achieve a specific displacement and hence controlling the current to achieve a certain displacement.

\subsection{Translation Micromirror}

A micromirror will be fabricated separately and will be bonded with the central ring of the actuator to develop a translating micromirror. Mirror plate without holes will have high optical quality. The mirror plate will be fabricated separately and bonded after the actuator is released to ensure there is no releasing hole in the mirror plate and also to achieve high mirror quality such as flatness. A mirror with $3.2 \mathrm{~mm}$ diameter and $0.2 \mathrm{~mm}$ thickness will be fabricated and bonded on the central ring of the actuator. The inner and outer diameters of the central ring of the designed actuator are $3 \mathrm{~mm}$ and $3.5 \mathrm{~mm}$. Therefore, the circumference of the $3.2 \mathrm{~mm}$ diameter mirror can be adhered to the central ring without exceeding the perimeter of the central ring outer surface as shown in Fig 3.5.

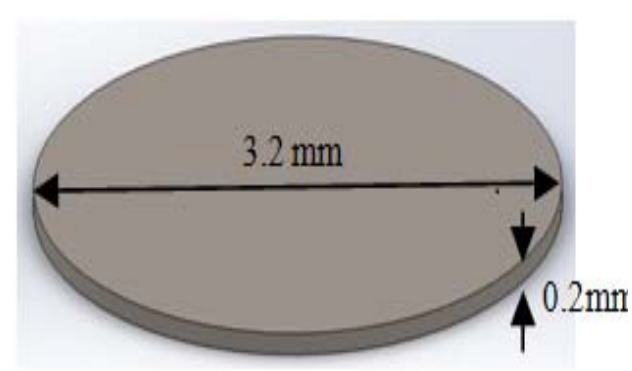

(a)

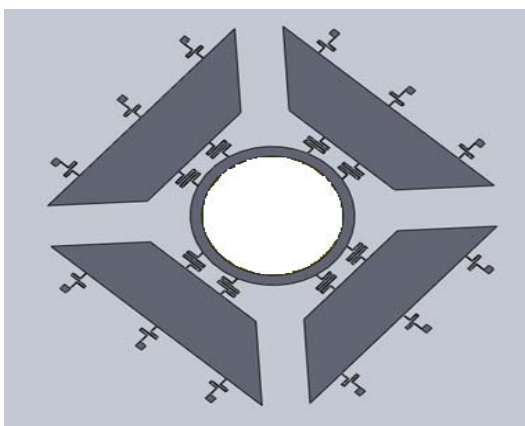

(b)

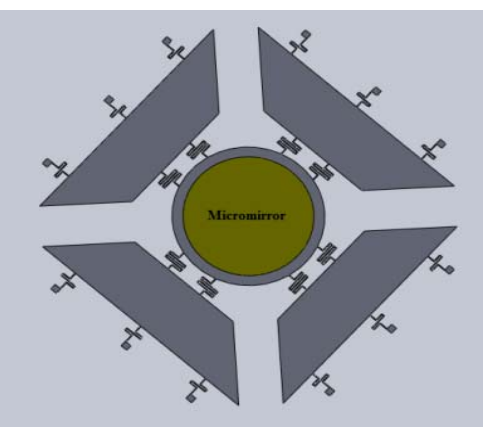

(c)

Figure 3.5: (a) Micromirror, (b) Actuator, (c) Translation Micromirror after Bonding

There are several different custom and commercial microfabrication processes to fabricate the micromirror and many different factors like cost, reliability and easiness of operation needs to be considered to choose the fabrication process. Commercial process like SOIMUMPS can be chosen for reliability and low cost in contrast with a customized process in order to ensure easy 
fabrication and achieve the required mirror quality. Polished wafer and simple microfabrication will be used for fabricating the mirror plate for the actuator. The mirror surface needs to be coated with a thin metal film to ensure high reflection before it is cut.

One possible way to bond the micromirror is adhesive bonding using glue. The glue could be epoxy, photoresist, or UV cured glue. A spin coating method can be used to coat the glue to coat the back side of the micromirror and then pressed over the central ring of the actuator. The large size of the mirror $(3.2 \mathrm{~mm}$ in diameter and $0.2 \mathrm{~mm}$ in thickness) makes the operation of the bonding possible and convenient. High stiffness of the released actuator will facilitate the bonding operation. It is difficult to quantify the required stiffness of the actuator for the bonding operation. It can only be determined through experimentation

\subsection{MEMS FTIRS}

One of the potential applications of the actuator designed in this thesis is in MEMS FTIRS with high sensitivity which will be used in the alcohol interlock system. There are three different design approaches for MEMS FTIRS as discussed in Chapter 2.2. Among these three design approaches, Michelson Interferometer based FTIRS is most popular due to its simple design and high sensitivity. Michelson interferometer based FTIRS design approach will be used for the design. Michelson interferometer based FTIRS has a translation micromirror which is moving forward and backwards to create the interferogram. The micromirror itself will be bonded to the actuator. This translation micromirror as shown in the Fig 3.5(c) will be used to develop MEMS FTIRS as shown in the Fig 1.5.

\subsection{Minimizing Tilt}

In Michelson interferometer based FTIRS, interference or fringe pattern is created due to the optical path difference between fixed and moving mirror. It is very important that the fixed and 
moving mirror is perfectly aligned during operation. If the mirror is tilted, the reflected light will be tilted and the fringe pattern will be distorted. Minimizing the tilt is one of the main challenges in case of MEMS FTIRS [21]. Tilting can occur for several different reasons. Tilting may exist due to the imperfection during microfabrication process. The imperfection occurs due to different chemical reactions during the microfabrication process. Two different fabrication processes have been used to fabricate translation micromirror to ensure both quality of large displacement and high mirror quality and then assembled together to construct translation micromirror. The assembling of such smaller scale component is always promising. Tilting may occur during the assembling of micromirror with the actuator. It will also be very critical to align the four solenoids exactly the same way above four trapezoidal plates as well as the location of the solenoids may not be exact. These will create tilting as well.

The actuator developed in this thesis has the capability of eliminating or decreasing mirror tilting. The mirror tilting minimization is achieved by determining and recording the applied current and mirror translation. Mirror translation has been realized by the conversion of the displacement of the top edge of the four trapezoidal plates. Mirror tilting occurs if the displacement of the top edge of the four actuator plates will not be the same. The four solenoids may not be exactly aligned with the trapezoidal plates and the four solenoids may not be the exactly same location of the trapezoidal plates. Therefore, the current requirements to achieve a certain displacement are different for four trapezoidal plates. The current vs. displacement has been recorded for each trapezoidal plate in order to predict the current requirement for each solenoid to achieve certain displacement. The same displacement can be achieved by the top edge of all trapezoidal plates by applying the respective current and hence minimize the mirror tilting. This type of control is open loop control which has the advantages of simplicity and 
stability but has no automatic feedback system. Another type of control system can also be used when a sensor will be used to give automatic feedback to each solenoid and hence apply that amount of current to realize same displacement. This system can provide more accuracy but is expensive and complicated. 


\section{Chapter 4: Design of Actuator}

The actuator has moving and fixed parts. The moving parts of the actuator consists of four trapezoidal plates, anchoring springs for anchoring the plates, connecting springs for connecting the trapezoidal plates and the central ring. The fixed part of the actuator consists of four solenoids placed above the four trapezoidal plates of the moving part. These solenoids will generate a magnetic force proportional to an applied current to displace the actuator.

\subsection{Design of the Moving Part}

The goal is to develop a high displacement MEMS magnetic actuator used in a FTIRS. The spectral resolution of the FTIRS depends on the mirror displacement. Spectral resolution is a measure of the ability of an instrument to distinguish spectral features that are close together that determines the number of data points in a spectrum. For example, a $4 \mathrm{~cm}^{-1}$ resolution spectrum contains a data point at every $4 \mathrm{~cm}^{-1}$ whereas, a $32 \mathrm{~cm}^{-1}$ spectrum contains a data at every $32 \mathrm{~cm}^{-1}$ [13]. High resolution is denoted by a small wave number whereas low resolution is denoted by large wave number.

The spectral resolution of FTIRS is the inverse of achievable Optical Path Difference (OPD)

Spectral Resolution $=1 / \mathrm{OPD}$

The optical path difference (OPD) between the two optical paths of a Michelson interferometer is given by Equation 4.2 .

$\mathrm{OPD}=2 \times$ displacement of moving mirror

The AutoCAD 2D diagram of the proposed magnetic actuator for the translating micromirror is shown in Fig 4.1. The overall dimension of the actuator is $10 \mathrm{mmx} 10 \mathrm{~mm}$. The design includes 
four trapezoidal plates located at the four corners which are symmetrical to each other. The larger side of the trapezoidal part is $6 \mathrm{~mm}$ and the shorter side is $3 \mathrm{~mm}$ and the distance between the two sides is $1.5 \mathrm{~mm}$. The larger side of the trapezoidal plate is fixed at the end through the three anchoring springs and the other side of the trapezoidal plate is connected to a central ring through two connecting springs. All the springs used in this design are serpentine type. The central ring is connected with the four trapezoidal plates with eight connecting spring. The outer diameter of the central ring is $3.5 \mathrm{~mm}$ and the inner diameter is $3 \mathrm{~mm}$. A micromirror will be attached on the center ring after fabrication.

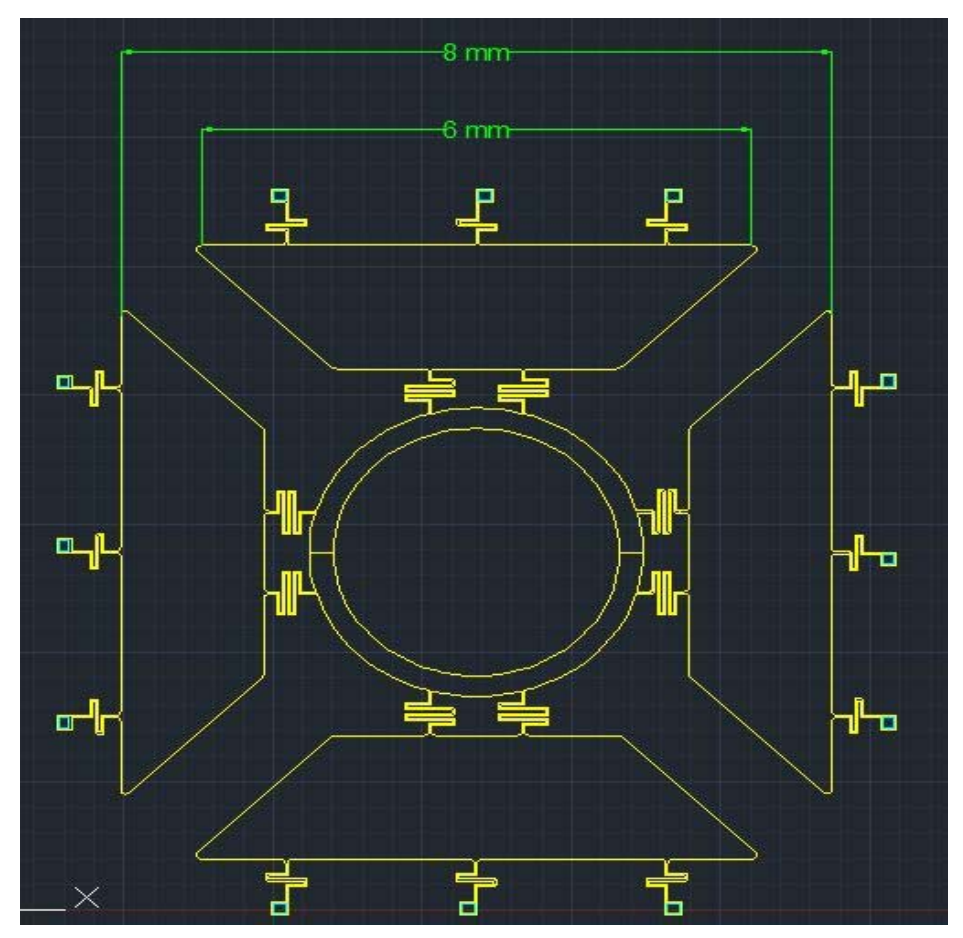

Figure 4.1: 2D Diagram of Proposed Magnetic Actuator

The detail dimensions of the trapezoidal plates, central ring, anchoring spring and connecting springs are shown in the Fig 4.2 


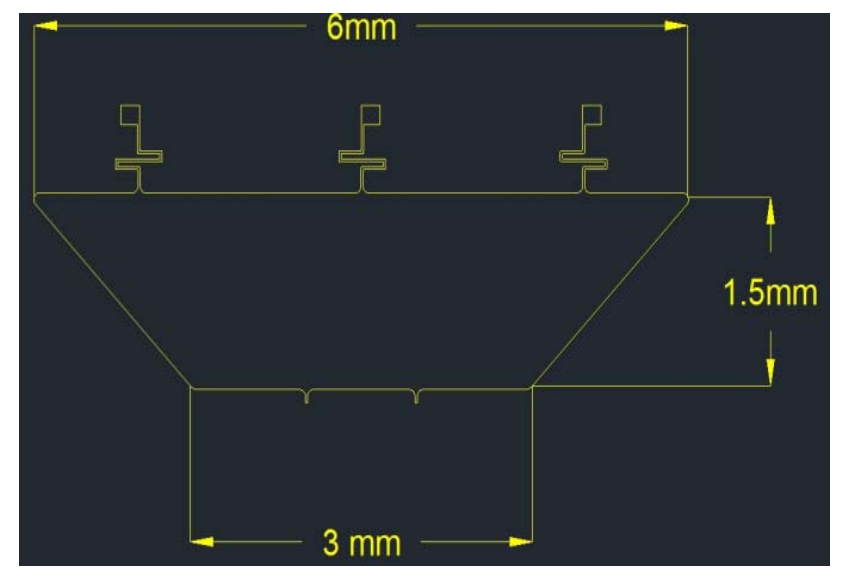

(a)

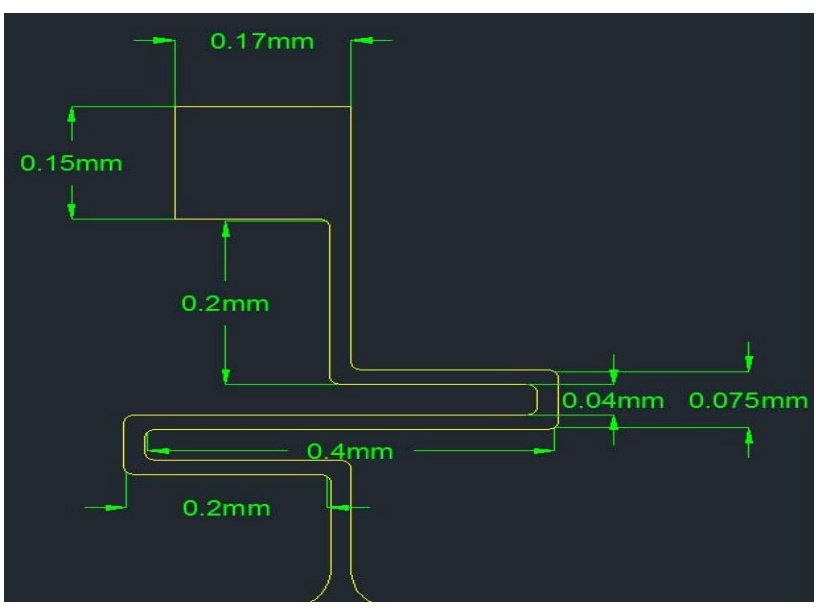

(c)

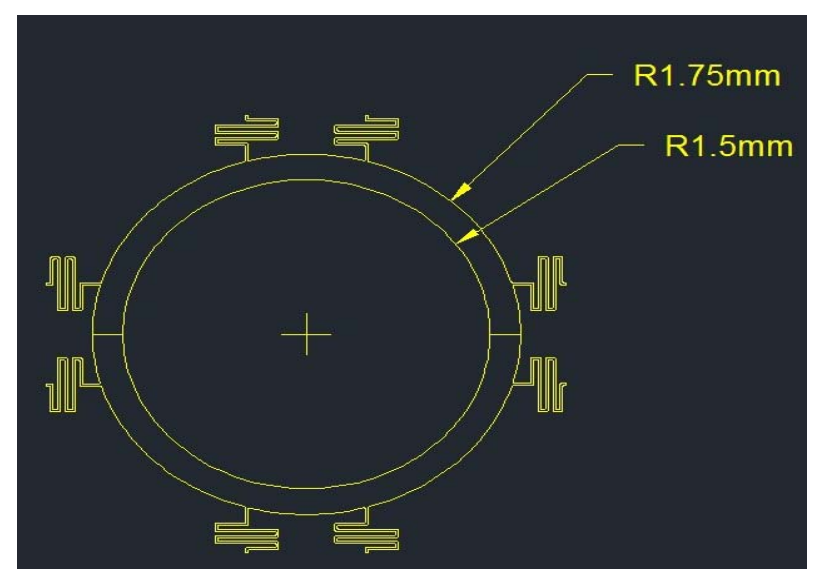

(b)

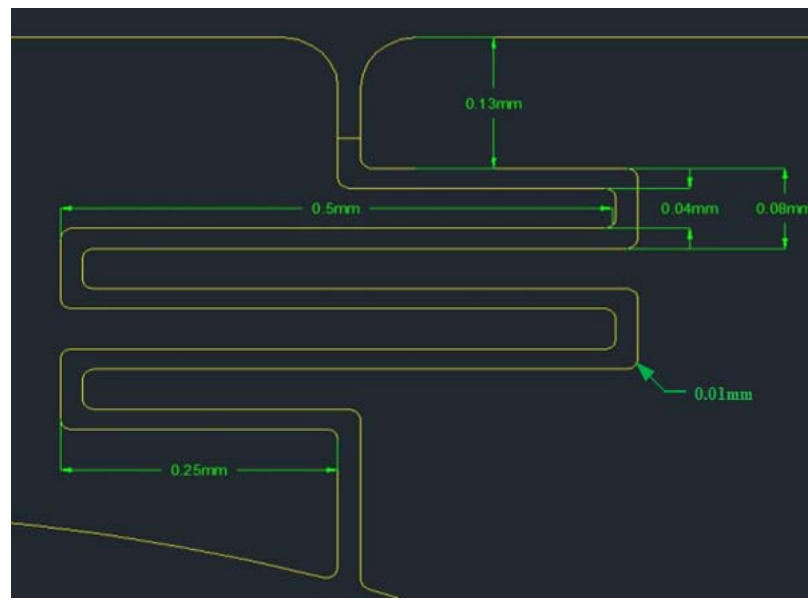

(d)

Figure 4.2: Detail Design of (a) Trapezoidal Plate, (b) Central Ring, (c) Anchoring Spring, (d) Connecting Spring

The MetalMUMPs fabrication process is used to fabricate this actuator. The Multi-User MEMS Processes (MUMPs) is a commercial program that provides cost-effective and reliable MEMS fabrication. It is also a mature and simple microfabrication process. MetalMUMPs is one commercial MUMPs process which is designed for general purpose electroplated nickel micromachining of MEMS components. Use of this commercial MetalMUMPs process will 
ensure low cost and reliable fabrication facility for the designed actuator over the other customized MEMS fabrication processes.

\subsection{Yield Strength Measurement:}

In mechanics of materials, yield strength is calculated from the stress-strain curve as shown in the Fig 4.3.

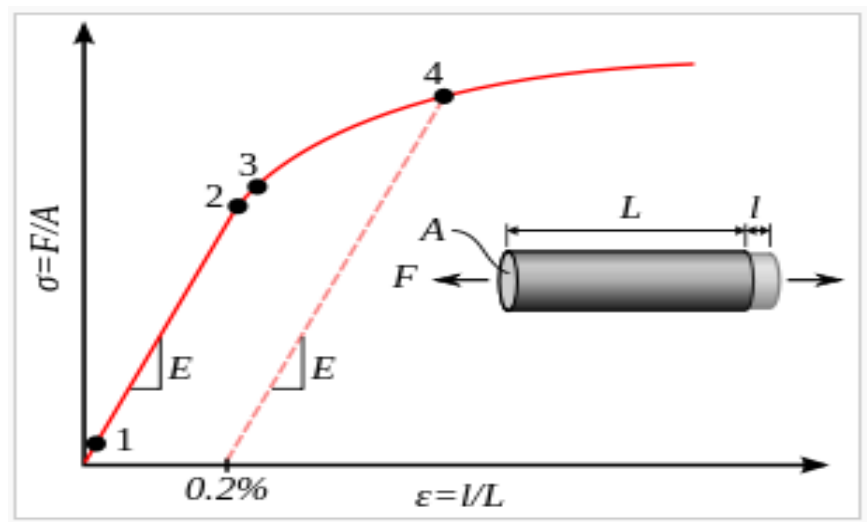

Figure 4.3: Typical Yield Behaviour for Non-ferrous Alloys. 1: True Elastic Limit, 2: Proportionality Limit, 3: Elastic Limit, 4: Offset Yield Strength [42]

There is no data available for yield stress of electroplated nickel fabricated in MetalMUMPs process. Zine-El-Abidine et al. mentioned that the yield strength of the electroplated nickel fabricated in MetalMUMPs process varies between 400MPa and 1000MPa. In 2002, Sharpe et al. performed measurement for yield strength of nickel as shown in Fig 4.4. This figure is used to determine the yield strength for the electroplated nickel as described below.

The curve in Fig 4.4 shows the stress vs. displacement. The procedure of calculating yield point is done using the stress-strain curve. In 2009, He et al. characterized the Young's modulus of the electroplated nickel fabricated using MetalMUMPs and found that the average Young's modulus for nickel film is $159 \mathrm{GPa}$. Young's modulus is the ratio of the stress and strain in the linear portion of a stress-strain curve. The corresponding stress for $0.002(0.2 \%)$ strain is $0.32 \mathrm{GPa}$ 
considering the young's modulus is 159GPa. The unannealed 0226_8a curve of Fig 4.4 has been used to measure the yield strength of the electroplated nickel.

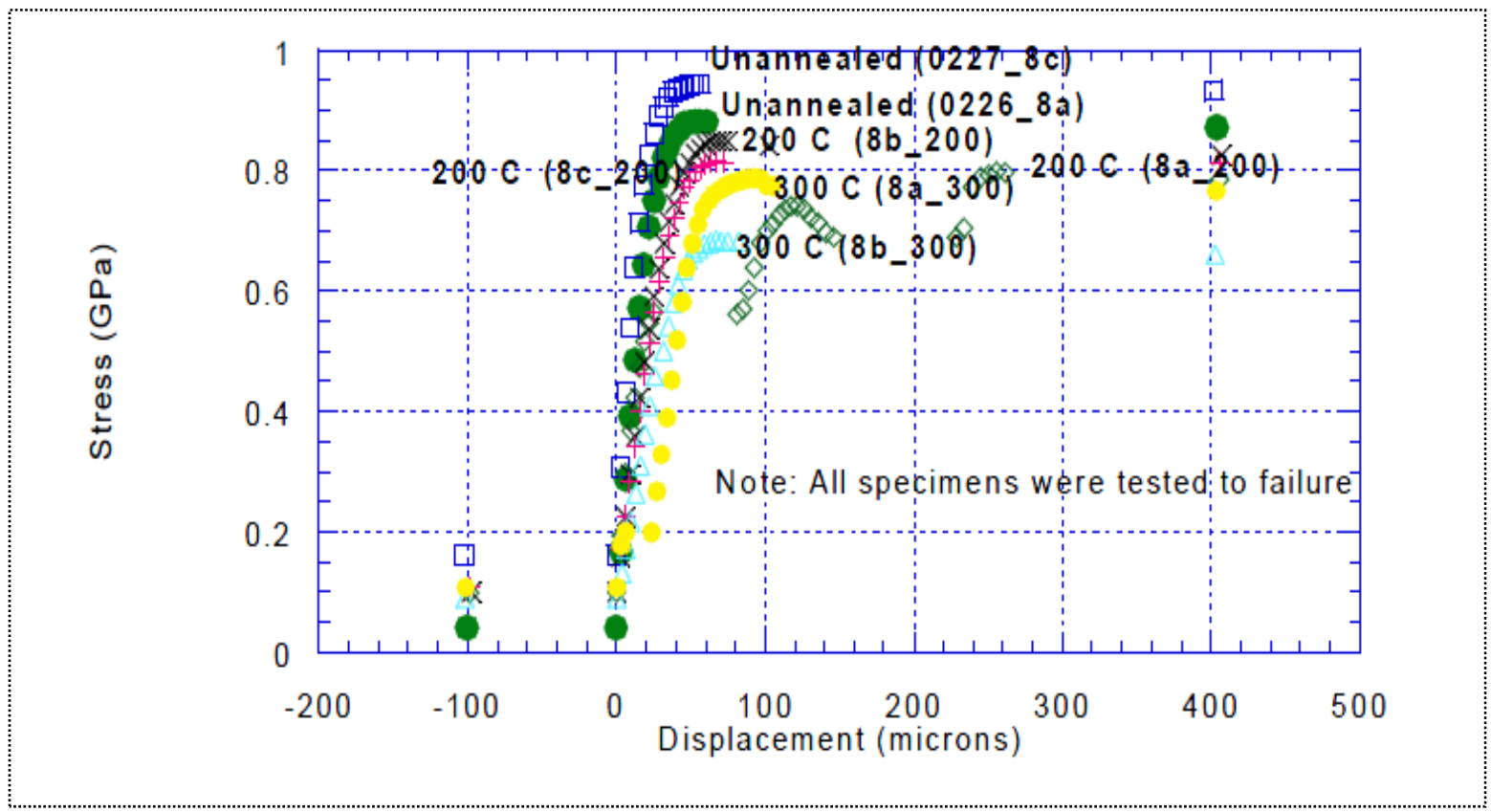

Figure 4.4: Yield Stress Measurement by Sharpe et al. [34]

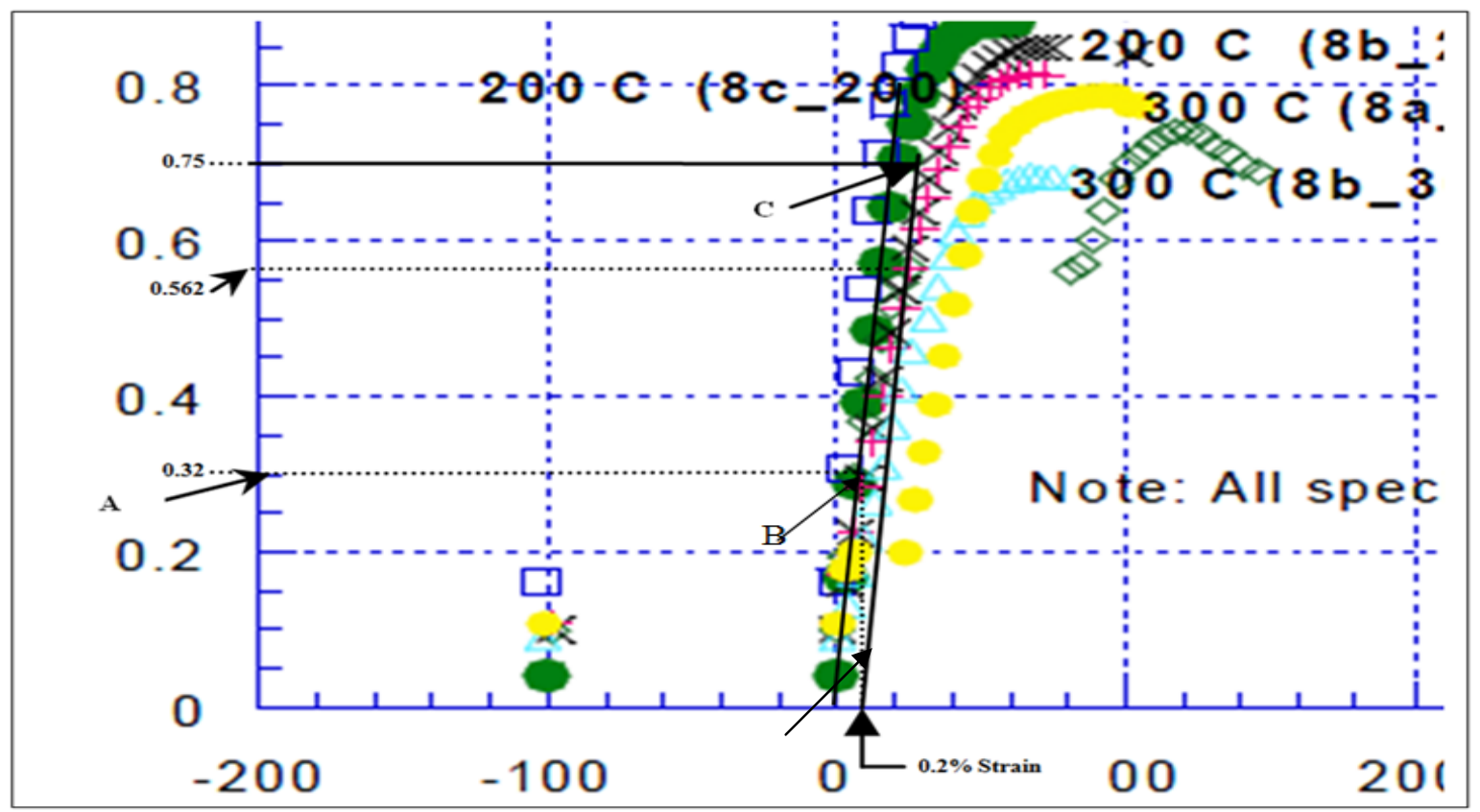

Figure 4.5: Measurement of Yield Stress for Electroplated Nickel 
Point A corresponds to $0.32 \mathrm{GPa}$ stress in Fig 4.5. If a horizontal line is drawn from point A, it will intersect with the stress-displacement curve (0226_8a) at point B. If a vertical line is drawn

from the intersection point $\mathrm{B}$, it will intersect the $\mathrm{X}$-axis at the point 0.002 or $0.2 \%$ of strain. If a line is drawn from this $0.2 \%$ strain point with the same slope of the linear portion of the stressdisplacement curve i.e. offsetting the stress-displacement curve linear portion will intersect the stress-displacement curve at point $\mathrm{C}$. The $\mathrm{Y}$ coordinate of point $\mathrm{C}$ is the yield strength of the electroplated nickel fabricated in MetalMUMPs process which is $0.75 \mathrm{GPa}$.

In order to ensure that a designed actuator does not yield in service, a safety factor 1.33 i.e. $75 \%$ [42] of the yield strength has been used. Therefore $562.5 \mathrm{MPa}(75 \%$ of $750 \mathrm{MPa})$ is the strength up to which nickel structure is safe.

\subsection{Simulated Performance of the Actuator}

The performance of the designed magnetic actuator has been simulated using CoventorWare, a FEA simulator dedicated to MEMS device design. The parameters presented in the embedded MetalMUMPs process in CoventorWare are used to simulate displacement and stress analysis of the designed actuator. The tetrahedral mesh type and 200 mesh size has been used to mesh the model. The displacement and VonMises stress under different load has been simulated. The simulated force vs. displacement and force vs. VonMises stress are shown in Fig 4.6 and Fig 4.7.

From the simulated force vs. stress curve it has been seen that the designed actuator experienced more than $600 \mathrm{MPa}$ of stress when $6 \mathrm{mN}$ load has been applied to the four trapezoidal plates of the actuator. From the Chapter 4.2 of yield strength measurement, it has been calculated that the 


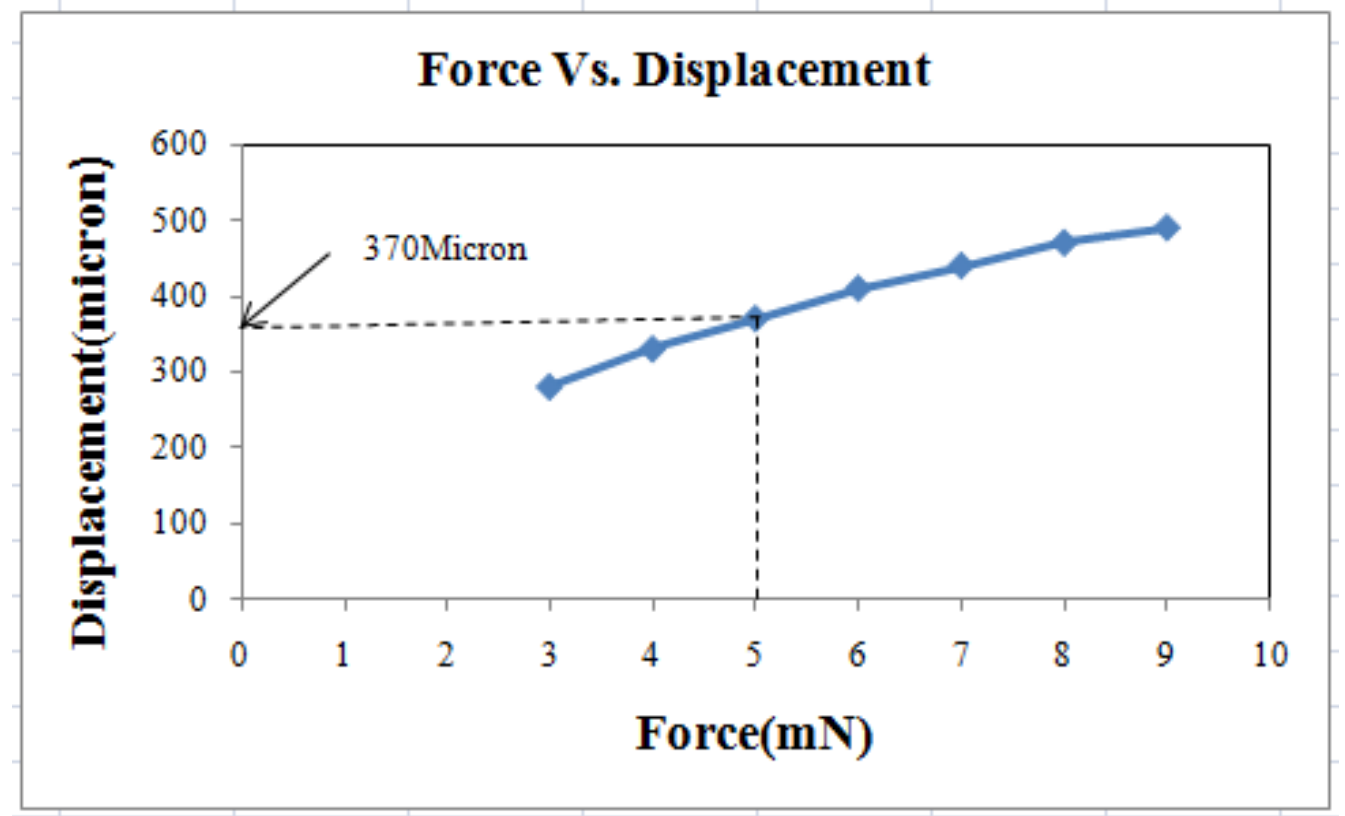

Figure 4.6: Simulated Force vs. Displacement

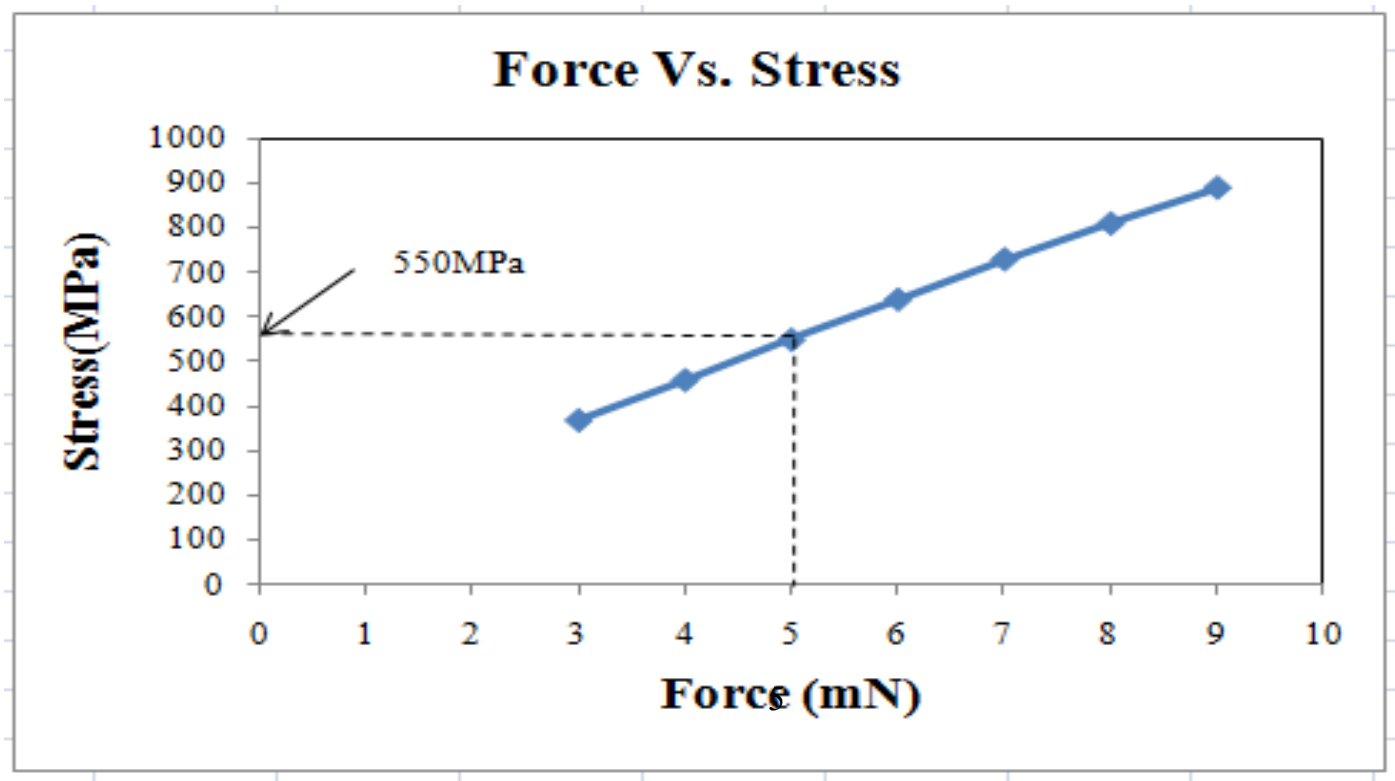

Figure 4.7: Simulated Force vs. VonMises Stress

yield strength for electroplated nickel fabricated in MetalMUMPs process is $562.5 \mathrm{MPa}$. Therefore, the applied load should be less than $6 \mathrm{mN}$. 
The simulation of the displacement and VonMises stress of the actuator under $5 \mathrm{mN}$ load is shown in Fig 4.8 and Fig 4.9.
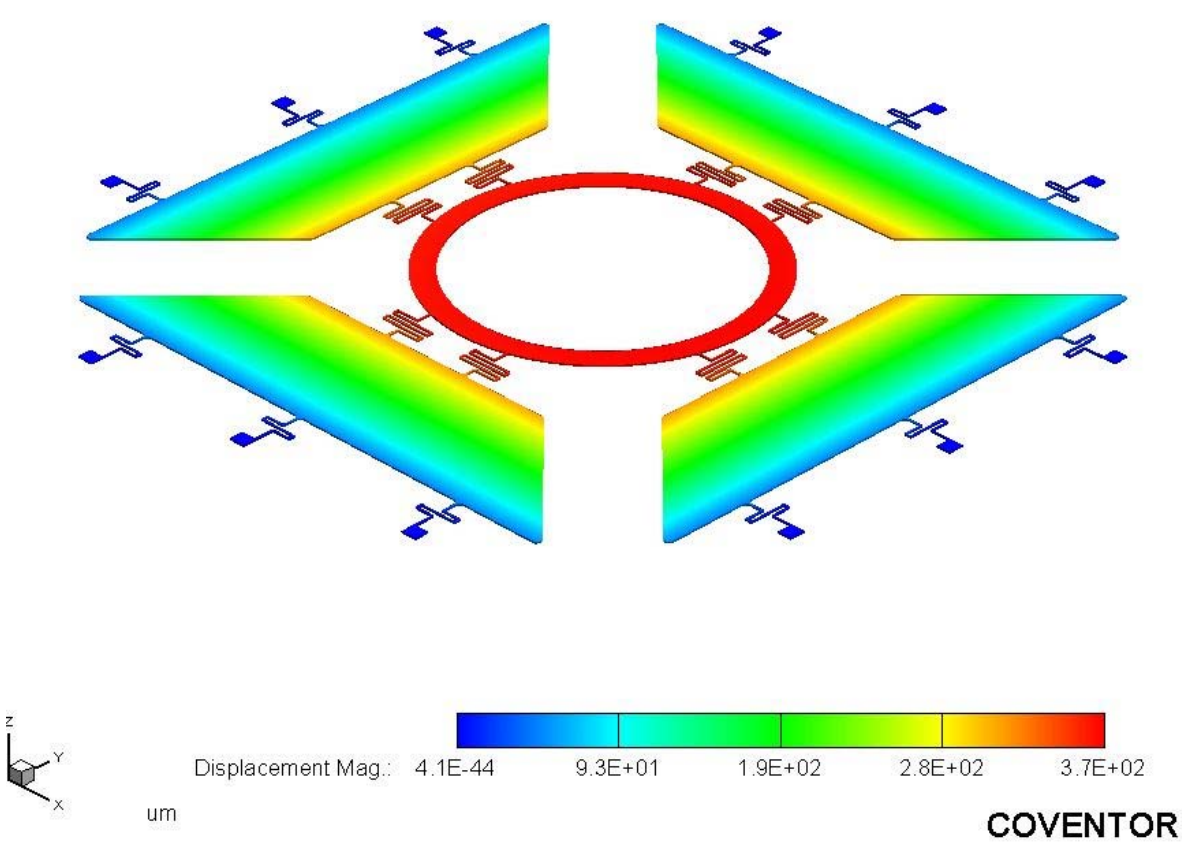

Figure 4.8: Displacement of Actuator @ 5mN Load
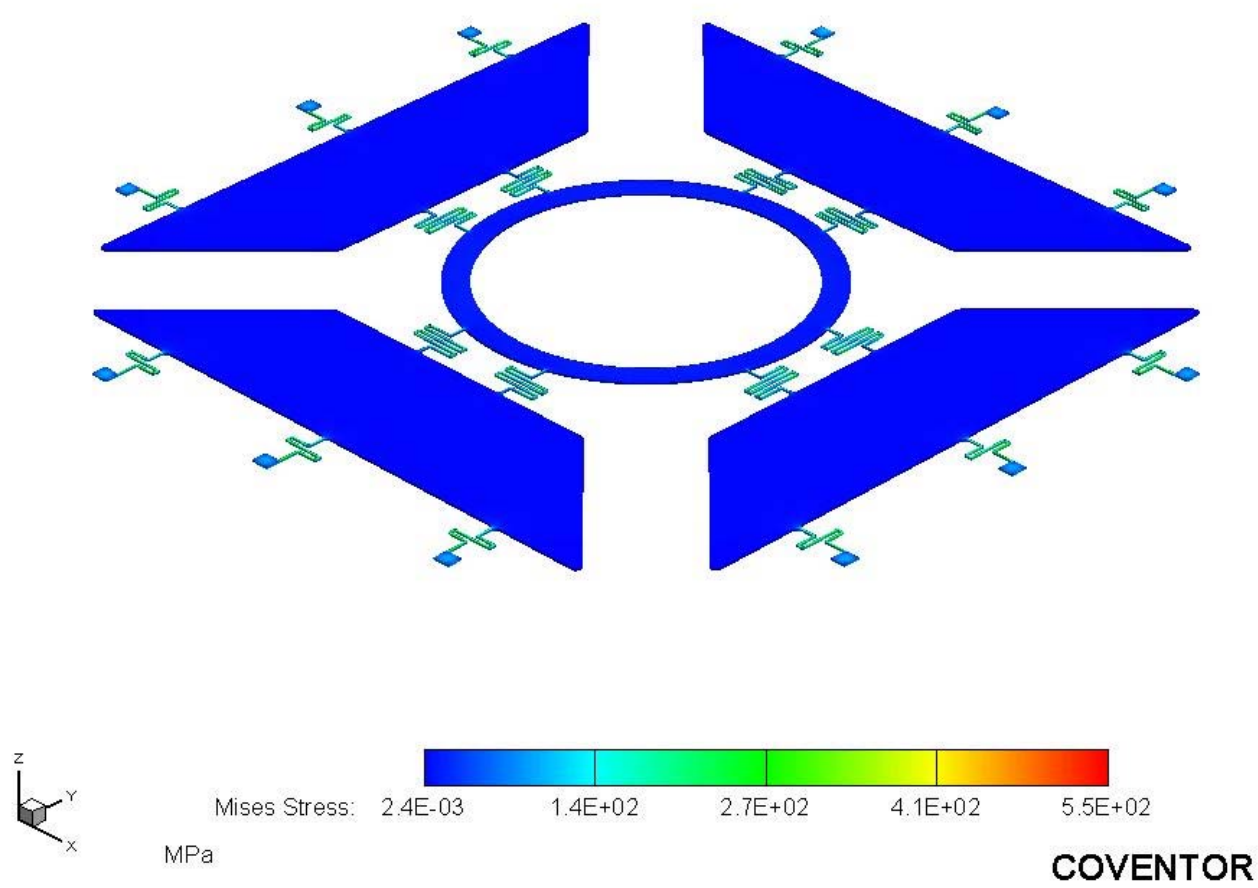

Figure 4.9: VonMises Stress of Actuator @ $5 \mathrm{mN}$ Load 
From the Fig 4.6 and Fig 4.7, it is found that the displacement of actuator under $5 \mathrm{mN}$ load is 370 micron and the VonMises stress is 550MPa. $5 \mathrm{mN}$ load is the total load applied on the four trapezoidal plates. It can be inferred that the actuator will work safely under $5 \mathrm{mN}$ load as the VonMises stress is smaller enough than the yield strength measured for electroplated nickel. The spectral resolution of the actuator can be estimated using the equation 4.2 and 4.3

$$
\mathrm{OPD}=2 \times \text { Displacement of moving mirror }=2 \times 370=740 \text { micron }
$$

$$
\text { Spectral resolution }=1 / \mathrm{OPD}=\left(1 / 740 \times 10^{4}\right) \mathrm{cm}^{-1}=13.5 \mathrm{~cm}^{-1}
$$

\subsection{Force and Magnetic Field Calculation}

The Permalloy beam is considered non-magnetized in the absence of external magnet as the remnant magnetization of the electroplated Permalloy is low. When current is applied to the solenoids, Permalloy materials of trapezoidal plates interact with the external magnetic field. Force acting on the trapezoidal plates can be calculated using Maxwell's pull force equation derived from the energy in B field equation.

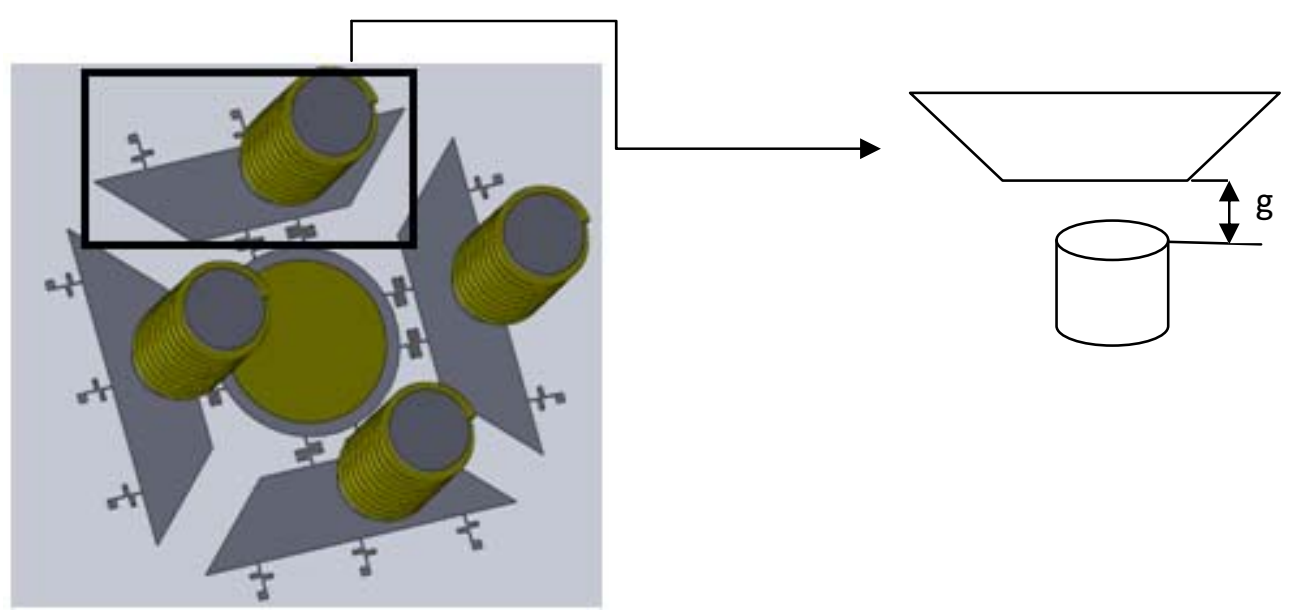

Figure4.10: Actuator and Solenoid Arrangement and its Cross-sectional View of a Single Trapezoidal Plate and Solenoid Arrangement 
Energy in Field B (FEB) equation [45] gives the energy density (joules per metre cubed). Assuming that the field inside the air gap is uniform, total energy can be calculated using the FEB equation by multiplying the volume of the magnetic field.

$$
W=\frac{B^{2}}{2 \mu_{0}} \times V
$$

The volume of the magnetic field (V) can be calculated [45] by multiplying the gap length (g) and the cross sectional area of the coil's core (A)

$$
V=g \times A
$$

From equation 4.4 and 4.5 ,

$$
W=\frac{B^{2}}{2 \mu_{0}} \times g \times A .
$$

The force on the trapezoidal part is given by the rate of change of energy with gap length

$$
\begin{gathered}
F=\frac{d W}{d g} \ldots \ldots \ldots \ldots \ldots \ldots \ldots \ldots \\
F=\frac{d}{d g}\left(\frac{B^{2}}{2 \mu_{0}} \times g \times A\right) \\
F=\frac{B^{2} A}{2 \mu_{0}}
\end{gathered}
$$

Where, $F$ is force (in Newton), $A$ is the cross section of the area of the trapezoidal plate in square meters, $B$ is the magnetic induction (magnetic flux density/magnetic flux) exerted by the solenoid and $\mu_{0}$ is the permeability of space, which equals $4 \pi \times 10^{-7} \mathrm{~T} \cdot \mathrm{m} / \mathrm{A}$. 
$5 \mathrm{mN}$ force is applied to all four trapezoidal plates. Therefore, the force in each trapezoidal plate is $1.25 \mathrm{mN}$. The area of the each trapezoidal plate is $6.75 \times 10^{-6} \mathrm{~m}^{2}$. Putting all these quantities in the equation 4.11 will provide the magnetic field requirement for each solenoid to generate $1.25 \mathrm{mN}$ force. The calculation is as follows:

$$
1.25 \times 10^{-3}=\frac{6.75 \times 10^{-6} \times B^{2}}{2 \times 4 \times 3.14 \times 10^{-7}}
$$

$$
\mathrm{B}=0.0027 \text { Tesla }
$$

Therefore, each solenoid needs to generate 0.0027 Tesla magnetic field. This calculation was performed assuming that the surface of the trapezoidal plate is parallel to the solenoids. Solenoid and trapezoidal plates are parallel at the beginning. When the current is applied to the solenoid, the trapezoidal plates will attract to the solenoid. Therefore, the distance between the free end of the trapezoidal plates and solenoids get closer and will require lower magnetic field.

\subsection{Design of the Fixed Part (Solenoid)}

The principle of a solenoid is based on electricity generated magnetic field and consists of a coil and a moving metal rod known as armature or plunger. When a current is passes through a coil, a very strong magnetic field is created in the solenoid. This field is then used to accelerate a plunger through the coil. The schematic of a solenoid is shown in Fig 4.11

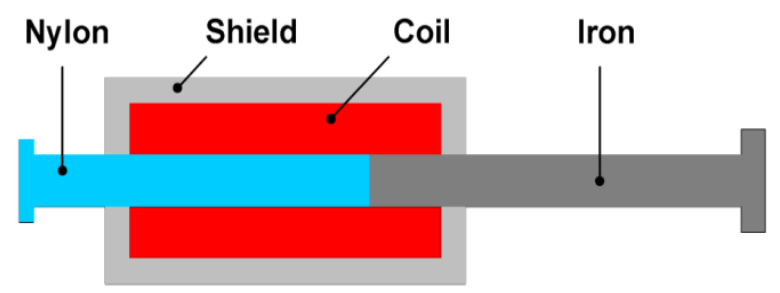

Figure 4.11: Schematic of Solenoid [37] 
The operation of solenoids is based on conversion of electrical energy into mechanical energy.

The magnetic field of the solenoid can be calculated using equation 4.14 [37]:

$$
B=\frac{\mu_{o} i N}{\sqrt{l^{2}+4 r^{2}}}
$$

Where, $\mathrm{B}$ is the magnetic field, in Teslas, at any point on the axis of the solenoid; $\mu_{0}$ is the permeability constant; $\mathrm{i}$ is the current in the wire, in amperes; $\mathrm{N}$ is the total number of turns of wire in the solenoid and 1 is the length and $r$ is the radius of the solenoid, in meters.

It has been calculated in the Chapter 4.4 that each solenoid needs to generate 0.0027 Tesla magnetic field. The summary of the parameters for the solenoid is shown in the Table 4.1

\section{Table 4.1 Solenoid Parameter}

\begin{tabular}{|c|c|c|c|c|}
\hline $\begin{array}{c}\text { Coil Diameter } \\
(\mathbf{m m})\end{array}$ & $\begin{array}{c}\text { Number of } \\
\text { Turns }\end{array}$ & $\begin{array}{c}\text { Coil Length } \\
(\mathbf{m m})\end{array}$ & Wire Geometry & $\begin{array}{c}\text { Wire Diameter } \\
(\mathbf{m m})\end{array}$ \\
\hline 2.5 & 50 & 4 & Round & 0.15 \\
\hline
\end{tabular}

The diameter of each solenoid has been chosen as $2.5 \mathrm{~mm}$. There will be four solenoids above four trapezoidal plates. The width of the each trapezoidal plate is $1.5 \mathrm{~mm}$. Therefore, an arrangement of four solenoids with $2.5 \mathrm{~mm}$ diameter each above four trapezoidal plates will leave enough space over micromirror to allow light passing through it. The wire dimension has been chosen as $0.15 \mathrm{~mm}$ round wire and the length of the coil has been chosen $4 \mathrm{~mm}$. Li et al. compared wires of different geometry for small solenoid in 2005 [35] and they used $2.5 \mathrm{~mm}$ and $1 \mathrm{~mm}$ diameter coil and 0.1 and $0.15 \mathrm{~mm}$ diameter wire. This confirms that it is possible to fabricate a solenoid consisting of $2.5 \mathrm{~mm}$ diameter coil and $0.15 \mathrm{~mm}$ diameter of winding wire. If the number of turn is 50 and the applied current $400 \mathrm{~mA}$ are considered, magnetic field can be calculated using equation 4.14 [37] as shown below: 


$$
\begin{gathered}
B=\frac{4 \times 3.14 \times 10^{-7} \times 0.4 \times 50}{\sqrt{\left(0.004^{2}+\left(4 \times 0.000075^{2}\right)\right.}} . \\
B=0.00627 \text { Tesla } \ldots \ldots \ldots \ldots
\end{gathered}
$$

The magnetic flux density generated by the solenoid is calculated 0.00627 Tesla using equation 4.10. The solenoids are placed $0.5 \mathrm{~mm}$ above the trapezoidal part. The calculated magnetic field density will decrease due to this air gaps between the solenoids and trapezoidal part. The magnetic field density considering the air gaps can be calculated using equation $4.17[45]$

$$
\begin{aligned}
& N i=B \frac{L_{\text {gap }}}{\mu_{0}} \ldots \ldots \ldots \ldots \ldots \ldots \\
& B=\frac{50 \times 0.4 \times 4 \times 3.14 \times 10^{-7}}{0.0005} \ldots \\
& \mathrm{B}=0.00502 \text { Tesla } \ldots \ldots \ldots \ldots
\end{aligned}
$$

The magnetic field density has been calculated 0.00502 Tesla considering air gap is $0.5 \mathrm{~mm}$. But the air gap between the free end of trapezoidal plates and solenoids will decrease when trapezoidal plates will start interacting with magnetic field.

It is found from the calculation in Chapter 4.4 that each solenoid needs to generate 0.0027 Tesla magnetic field to generate $1.25 \mathrm{mN}$ force. The total force applied on the four trapezoidal plates is $5 \mathrm{mN}$ i.e. each plate is subjected to $1.25 \mathrm{mN}$ force. In Chapter 4.3 , it has been modeled that 370 micron displacement can be achieved when $5 \mathrm{mN}$ force is applied on the four trapezoidal plates. Therefore, it can be inferred that the 370micron displacement can be achieved using the proposed solenoid when $400 \mathrm{~mA}$ current is applied to each solenoid. 


\subsection{Working Frequency:}

The actuator mirror assembly has to be operated in static mode to provide linear motion instead of oscillatory motion at resonant mode. The frequency of the moving mirror will be less than 5 Hz. Therefore, the natural frequency should be more than the working frequency to ensure static mode operation. The natural frequency for the actuator without mirror has been simulated using Coventorware and found that the natural frequency is more than ten times than the working frequency. Then actuator plate assembled with a 200micron mirror plate has also been simulated and natural frequency is found more than ten times of the operated frequency. Therefore, it can be inferred that translation micromirror consisting of the mirror plate of 200micron thickness is capable of producing required displacement at static mode. The first three mode of natural frequency is shown in Table 4.1

Table 4.2: First Three Mode of Natural Frequency

\begin{tabular}{|c|c|c|}
\hline \multirow{2}{*}{ Mode } & \multicolumn{2}{|c|}{ Natural Frequency(Hz) } \\
\cline { 2 - 3 } & Without Mirror & With Mirror \\
\hline 1 & 290.14 & 158.65 \\
\hline 2 & 590.35 & 454.37 \\
\hline 3 & 590.75 & 454.69 \\
\hline
\end{tabular}

The deformed shape of the actuator for the first mode of the natural frequency with and without mirror is shown in Fig 4.12 and 4.13. 

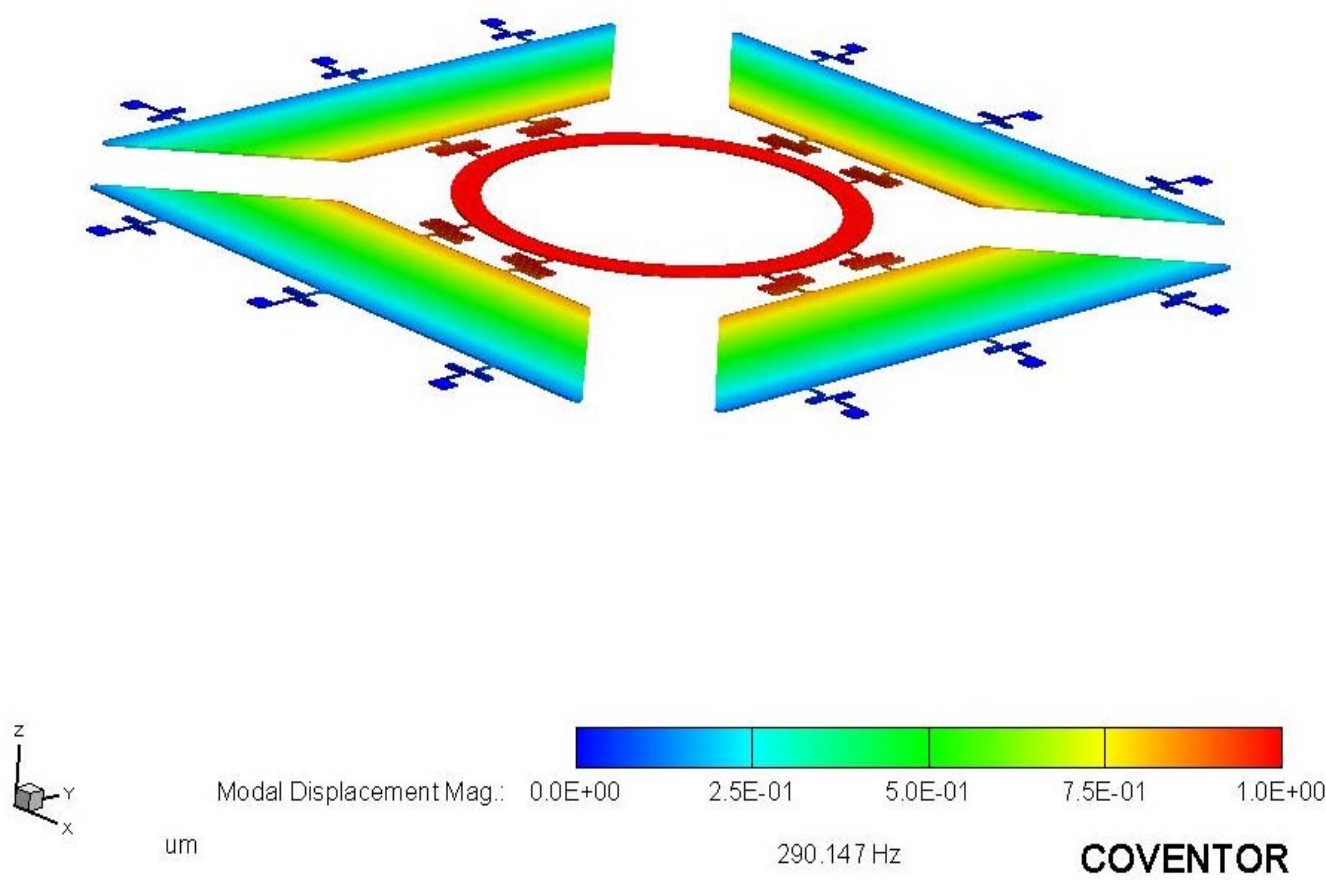

Figure 4.12: 10First Mode of Natural Frequency (without mirror)

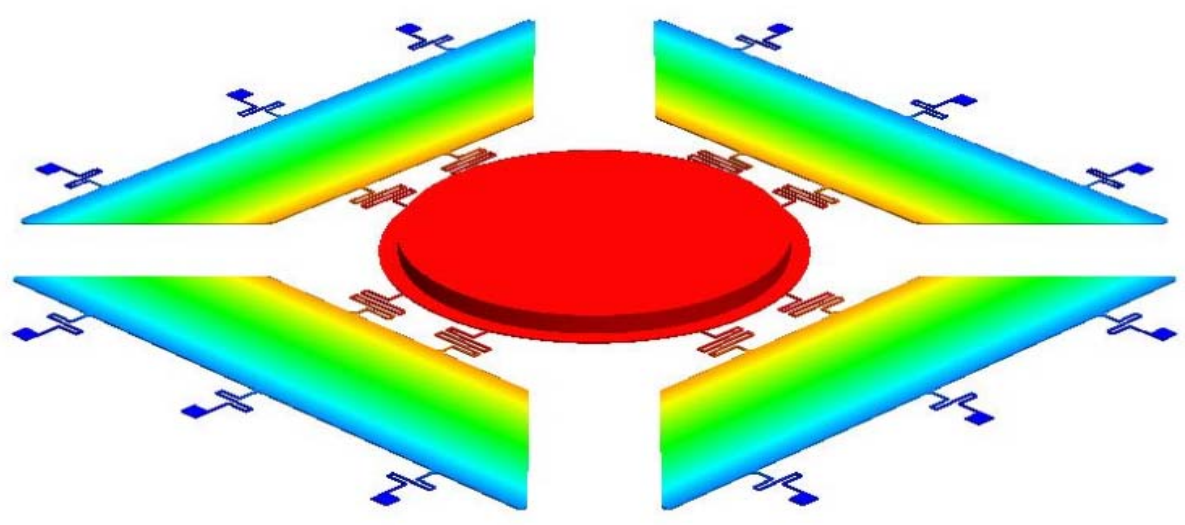

$\mathbb{R}^{2}$

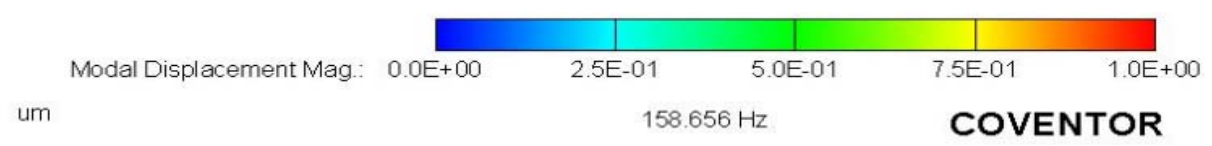

Figure 4.13: First Mode of Natural Frequency (with mirror) 


\subsection{Verification of bonding operation}

Experimental tests by another student showed that the current prototype of the moving part of the actuator can survive during the bonding operation. It ensures that the approach of developing a translation micromirror using two separate fabrication process for actuator and micromirror is feasible.

\subsection{Improvement of the existing design}

The main objective of this thesis was to design a high displacement MEMS magnetic actuator which will be used in MEMS FTIRS to realize high sensitivity in alcohol interlock system. It has been found that designed actuator is capable of achieving 370 micron of displacement. The highest displacement achieved by FTIRS is 600 micron developed by Block MEMS LLC. But the actuator used in that FTIRS is a thermal actuator. Thermal actuator has disadvantages of use in the long run as polysilicon break at high temperature as well as thermal actuator is less actuator and less reliable. The designed actuator use two microfabrication processes to achieve both features of high displacement and high mirror quality. Commercial MetalMUMPs process has been used to fabricate magnetic actuator and silicon wafer is used to fabricate the micromirror and then assembling together. The displacement achieved by the designed actuator is less than the available FTIRS. But the designed FTIRS is predicted to perform better as both high displacement and high mirror quality has been incorporated together comparing to other available FTIRS. Determining the current for each solenoid for a specific displacement and hence controlling the current based on that system will decrease the tilt of the mirror which improves the overall performances of the FTIRS.

It has been found that the structure is too stiff to produce larger displacement than 370micron. Larger displacement can be achieved by decreasing the stress. In has been found from the stress 
simulation that the concentration of stress is in the corner of the springs as shown in Fig 4.14. Redesigning the spring stiffness can decrease the stress and can improve the performance of the designed actuator.

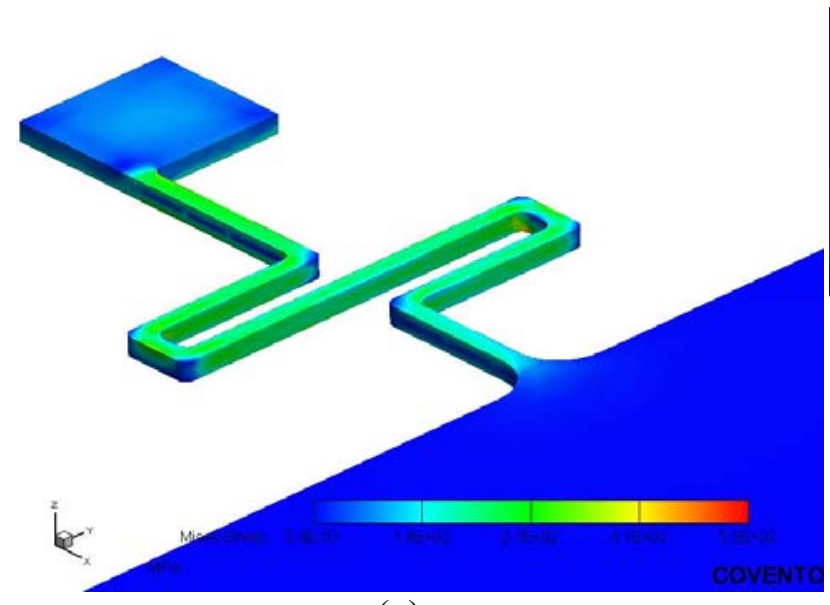

(a)

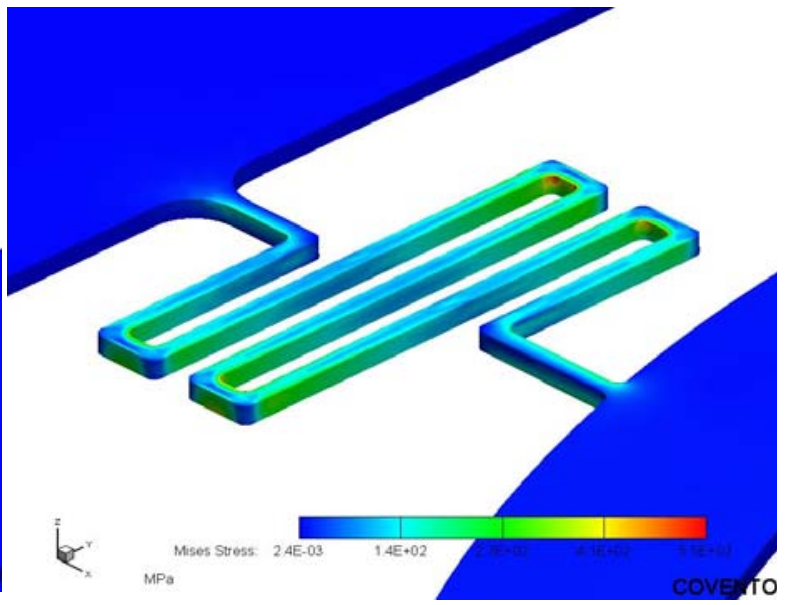

(b)

Figure 4.14: Stress Concentration at the Corner of the Spring: (a) Anchoring Spring, (b) Connecting Spring

Box spring and serpentine type spring are the two types of spring used in MEMS components. The layout of these two types of spring is shown in the Fig 4.15 (a) and (b)

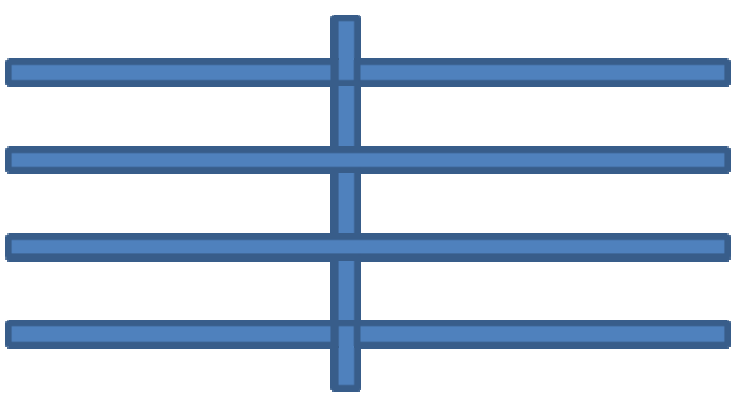

(a)

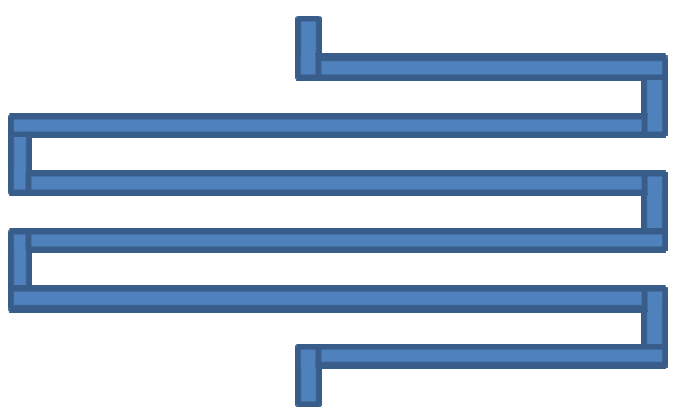

(b)

Figure 4.15: Layout of Springs (a) Box Spring, (b) Serpentine Spring

Box type springs are consisting of number of boxes connected together. These types of springs are stiffer and are not capable of exhibit sideways movement at the ends. On the other hand, 
serpentine type springs are consisting of number of bars connected on alternating ends. These types of spring are capable of exhibiting sideways movement and are less stiff. Therefore, serpentine type springs are used to acquire more displacement.

Fillets are used at the corner of the spring to distribute the load over larger areas to reduce stress concentration at the corner. The size of the fillet in the spring of the actuator is $0.01 \mathrm{~mm}$ as shown in Fig 4.2(c) and (d). Increasing the size of the fillet will reduce the stress concentration at the corner.

Commercial MetalMUMPS is used to fabricate the prototype. The thickness of the metal (Nickel) layer cannot exceed 20 micron in case of MetalMUMPS as shown in Table 5.1. Therefore, it is not possible to design a very large spring. But number of turns and other attributes of the spring can be adjusted in order to reduce stress concentration. There are two turns in case of anchoring springs and one turn in case of connecting springs as shown in Fig 4.2 (c) and (d) . Increasing the number of turns either in anchoring springs or connecting springs or both will help to reduce the stiffness as well as stress.

In serpentine type springs, there are bars which are connected at alternative ends to provide sideways movement. The size of the bar of the spring has a huge effect on the stiffness. Increasing the length of the bar will also help to reduce the stiffness and hence reduce stress. The gap between the two bars of the spring can also plays an important role on the stiffness. Increasing the gap will also decrease the stiffness.

Another modification to improve the performance of the overall system is redesigning the central ring where micromirror will be assembled. The size of the center ring needs to be decrease in order to leave more space for the spring with more number of turns. As stated earlier, increasing 
the number of turns in the spring will make the system less stiff which will help to achieve more displacement. In addition to decreasing the size of center ring, a center structure is also needed for the ring. Otherwise there is chance of touching the substrate when micromirror will be glued on this ring. 


\section{Chapter 5: Prototype Fabrication}

MEMS magnetic actuator designed in this thesis has been fabricated using commercial MetalMUMPs microfabrication process. Microfabrication is a special micromachining process used to fabricate MEMS component and is different from the traditional machining and manufacturing process and similar to the IC (Integrated Circuit) manufacturing process. There are several different microfabrication processes available. Some are customized others are commercial. Customized process has the advantages of manufacturing components of any desired attributes, whereas commercial process has some specific criterion but has the advantages of low cost, reliability and higher fabrication success rate. The Multi-User MEMS Processes or MUMPs are commercial MEMS component fabrication process. MEMSCAP offers three standard processes as part of the MUMPs program: PolyMUMPs, a three-layer polysilicon surface micromachining process; MetalMUMPs, an electroplated nickel process; and SOIMUMPs, a silicon-on-insulator micromachining process. The MEMS magnetic actuator in this thesis is fabricated using MetalMUMPs process.

\subsection{MetalMUMPs Process Steps}

Electroplated Permalloy layer is necessary for the designed actuator in order to realize the magnetic interaction at the presence of external magnetic field. Therefore, MetalMUMPs fabrication process has been chosen to fabricate the actuator. MetalMUMPs is a commercial micromachining process where nickel is used as the electroplated metal. The process is facilitated by MESCAPE. The process consists of the following steps [46]:

1. MetalMUMPs process is started with a $\mathrm{N}$-type(100) silicon with a $2 \mu \mathrm{m}$ oxide layer(isolation oxide) and deposition of phosphosilicate glass (PSG) layer known as Oxide1 as shown in Fig 5.1. It is then coated with UV sensitive photoresist and lithographically patterned with the first 
level mask known as OXIDE1. The area of the photoresist which are exposed to the UV is removed and wet etching is used to remove the unnecessary sacrificial layers.

2. The second step is the deposition of a $0.35 \mu \mathrm{m}$ layer of silicon nitride known as NITRIDE1and $0.7 \mu \mathrm{m}$ layer of Poly Silicon known as Poly as shown in Fig 5.1. The wafer is then again coated with photoresist and patterned with the 2nd mask level known as POLY. Reactive Ion Etching (RIE) is used to remove the unwanted polysilicon and photoresist is removed after the etch is completed. A second $0.35 \mu \mathrm{m}$ layer of silicon nitride known as Nitride2 is then deposited and coated with photoresist and patterned with third mask level known as NITROHOLE. RIE etching is used to remove both Nitride1 and Nitride2 from the patterned area and photoresist is removed when etch is completed.

3. The third step is patterned the wafer with the fourth mask level METANCH to create a channel for anchoring the metal layer. A thin metal layer (Anchor Metal) consisting of 10nm Cr and $25 \mathrm{~nm}$ Pt is deposited and a liftoff process is used to remove the photoresist and leave the metal layer as shown in Fig 5.1.

4. The fourth step is to create a plating base of $500 \mathrm{~nm} \mathrm{Cu}$ and $50 \mathrm{~nm}$ Pt. The wafer is covered with photoresist and lithographically patterned with the fifth mask level METAL to form a patterned stencil for the electroplated metal layer. Then the nickel is electroplated into the patterned stencil with a thickness of $20 \mu \mathrm{m}$ followed by $0.5 \mu \mathrm{m}$ gold layer on the top of the metal layer as shown in Fig 5.1. The electroplated nickel with the gold layer together creates the metal layer.

5. Finally the wafer is released in two steps. First, is to chemically stripped the plating base and then remove sacrificial layers using $49 \% \mathrm{HF}$ solution. Then a protective coating is applied and the wafers are diced, cleaned, sorted and shipped to customer. 
The graphical presentation of the METALMUMPS process is as follows:

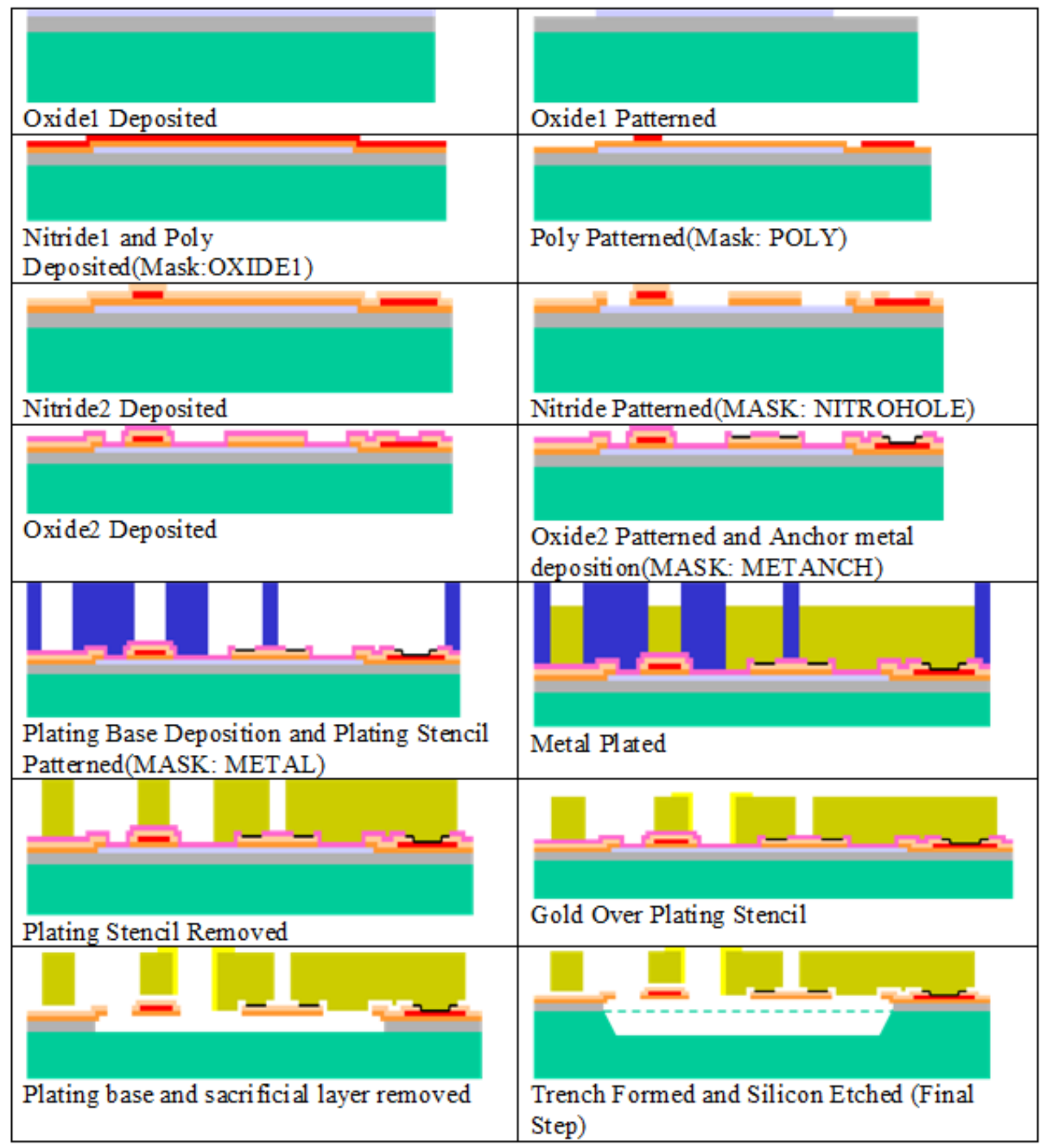

\begin{tabular}{|l|l|l|l|l|l|l|l|l|l|}
\hline & Substrate & & Oxide 1 & & Poly & & Oxide 2 & & Metal \\
\hline & Isolation Oxide & & Nitride 1 & & Nitride 2 & & Anchor Metal & & Sidewall Metal \\
\hline
\end{tabular}

Figure 5.1: Graphical Presentation of MetalMUMPs [46] 


\subsection{MetalMUMPs layers and materials properties}

MetalMUMPs process has different layers and some design rules which has to be followed properly in order to ensure successful fabrication. The design rules are a set of requirements that defines the minimum feature sizes and spaces for all levels and minimum overlap and spacing between relevant levels. The purpose of the rule is to ensure the successful fabrication. These rules are developed through process development and the experience of the MEMSCAP staff. Some rules are mandatory which must be followed whereas some are not. The minimum line widths and spaces are mandatory rules. Mandatory rules are given to ensure that all layouts will remain compatible with MEMSCAP lithographic process tolerances. The violation of the design rule may result in missing, undersized, oversized or fused features. Minimum overlap (enclosure, cut-in and cut-out rules) requirements reduce the effect of large topographies and prevent unnecessary etching of underlying layers whereas minimum spacing between levels guarantees that features of two different levels can be delineated by photolithography and etch [46]. The summary of the design rule for MetalMUMPs process are shown in Table 5.1 and 5.2

Table 5.1: Layer names, thicknesses and lithography levels (from MetalMUMPs Manual)[46]

\begin{tabular}{|c|c|c|c|}
\hline $\begin{array}{c}\text { Material } \\
\text { Layer }\end{array}$ & $\begin{array}{c}\text { Thickness } \\
(\boldsymbol{\mu m})\end{array}$ & $\begin{array}{c}\text { Lithography } \\
\text { Level Name }\end{array}$ & Lithography Level Purpose \\
\hline Isolation Oxide & 2.0 & & Define trench; release Nitride \\
\hline Oxide 1 & 0.5 & OXIDE1 & Pattern Poly \\
\hline Nitride 1 & 0.35 & POLY & $\begin{array}{c}\text { Define areas where is removed. Define } \\
\text { trench. Open hole for electrical contact } \\
\text { between metal and Poly }\end{array}$ \\
\hline Poly & 0.7 & NITRHOLE & $\begin{array}{r}\text { Open hole to anchor Metal to Nitride or } \\
\hline \text { Nitride 2 }\end{array}$ \\
\hline Oxide 2 & 1.1 & METANCH &
\end{tabular}




\begin{tabular}{|c|c|c|c|}
\hline $\begin{array}{c}\text { Material } \\
\text { Layer }\end{array}$ & $\begin{array}{c}\text { Thickness } \\
(\boldsymbol{\mu m})\end{array}$ & $\begin{array}{c}\text { Lithography } \\
\text { Level Name }\end{array}$ & Lithography Level Purpose \\
\hline Anchor Metal & 0.035 & & $\begin{array}{c}\text { Poly. Open holes for thin metal traces of } \\
\text { Anchor Metal }\end{array}$ \\
\hline Plating Base & 0.55 & & Pattern Metal \\
\hline Metal & 20.5 & METAL(HOLM) & \\
\hline
\end{tabular}

Table 5.2: MEMSCAP level name, CIF and GDSII' ${ }^{T M}$ level designation, and nominal and minimum features and spaces for each level.(MetalMUMPs Manual)[46]

\begin{tabular}{|c|c|c|c|c|}
\hline $\begin{array}{c}\text { Mnemonic } \\
\text { Level Name }\end{array}$ & CIF Level Name & $\begin{array}{c}\text { GDS Level } \\
\text { Number }\end{array}$ & $\begin{array}{c}\text { Minimum } \\
\text { Feature } \\
(\boldsymbol{\mu m})\end{array}$ & $\begin{array}{c}\text { Minimum } \\
\text { Space }(\boldsymbol{\mu m})\end{array}$ \\
\hline OXIDE1 & OX1 & 10 & 20 & 20 \\
\hline POLY & POLY & 20 & 5 & 5 \\
\hline NITRHOLE & NITR & 30 & 5 & 5 \\
\hline METANCH & ANCH & 40 & 50 & 10 \\
\hline METAL & METL & 50 & 8 & 50 \\
\hline GOLDOBP & OVP & 60 & 50 & 5 \\
\hline HOLEP & HOLP & 21 & 5 & 8 \\
\hline HOLEM & HOLM & 51 & 8 & 5 \\
\hline
\end{tabular}

The minimum thickness of different layers of MetalMUMPS process are stated in Table 5.1. The minimum thickness for isolation oxide is $2 \mu \mathrm{m}$, Oxide1 is $0.5 \mu \mathrm{m}$, AnchorMetal is $0.035 \mu \mathrm{m}$, Metal is $20.5 \mu \mathrm{m}$ including the gold layer. The component fabricated in MetalMUMPS process cannot exceeds the thickness as stated in Table 5.1. The design rules i.e. CIF and GDS level name, minimum features and minimum thickness of the material layers are shown in the Table 5.2. The minimum features for OXIDE1, METANCH, METAL and HOLEM are $20 \mu \mathrm{m}, 50 \mu \mathrm{m}, 8 \mu \mathrm{m}$ and 
$8 \mu \mathrm{m}$. These features have to be strictly followed in order to ensure successful fabrication. The AUTOCAD diagram sent to MEMSCAPE for prototype fabrication using MetalMUMPS process is shown in Fig 5.2. All rules of Table 5.1 and 5.2 have been followed.

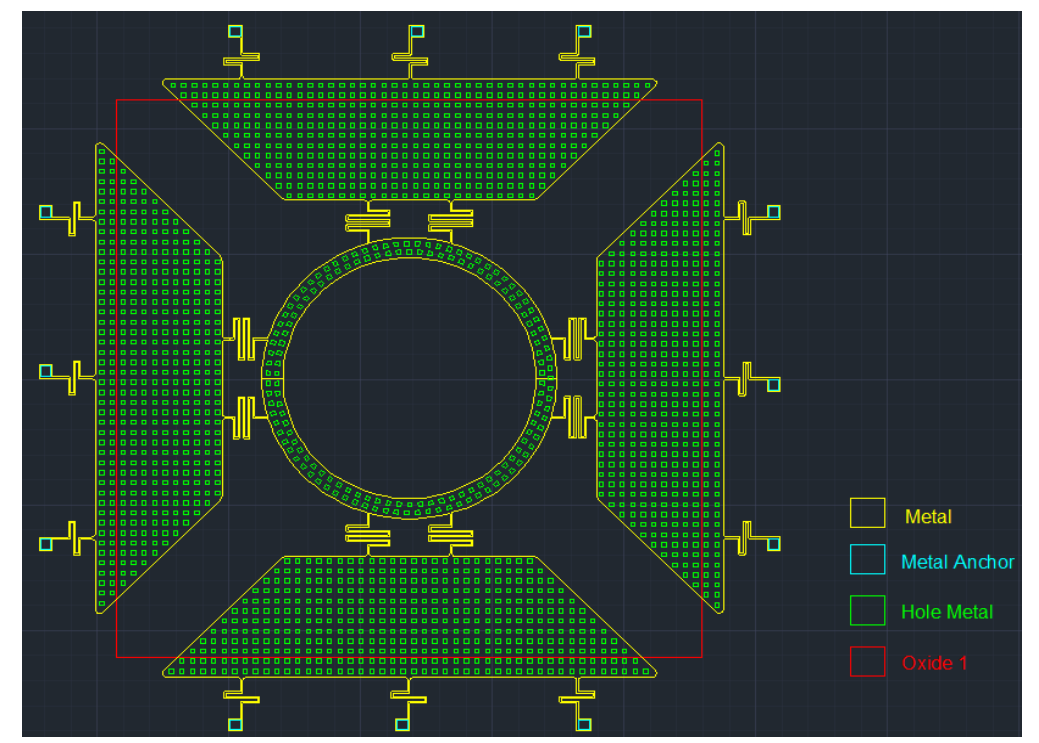

Figure 5.2: Actuator Design in AUTOCAD

\subsection{MetalMUMPs process simulations}

The Coventorware software has been use to generate the 3D model of the actuator using actual MetalMUMPs process simulation. The rules and parameters described in Table 5.1 and 5.2 have been used. This software uses the real time simulation of the MetalMUMPs process and generates a 3D model of the AutoCAD drawing. Like the real MetalMUMPs process, it also starts with a substrate of N-type silicon as shown in Fig 5.3(a) followed by a deposition of a $0.5 \mu$ sacrificial phosphosilicate glass (PSG) layer known as Oxidelin Fig 5.3(b) and deposition of Nitride1 as shown inFig 5.3(c). No Poly and Nitride2 layer were used in this fabrication. Then the nickel is electroplated into the patterned stencil with a thickness of $20 \mu \mathrm{m}$ as shown in Fig 5.3(d) and a $0.5 \mu \mathrm{m}$ gold layer is immediately deposited on the top of the metal layer and forms the Metal layer as shown in Fig 5.3(e). The final 3D model of the designed actuator generated by 
the CoventorWare is shown in Fig 5.3(f). The holes which are needed for the successful release of the metal structure are not included in the simulation due to the huge time requirement for processing. The graphical representation of the MetalMUMPs process simulation is shown in the figure below:

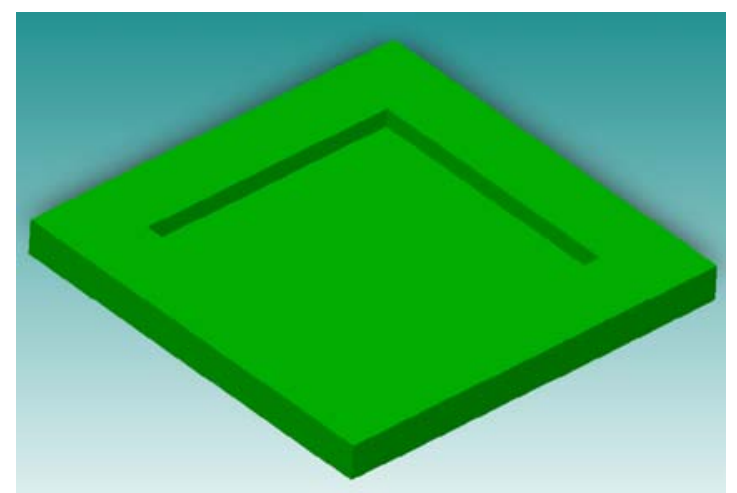

(a)Starting Wafer (N-type 100 Silicon)

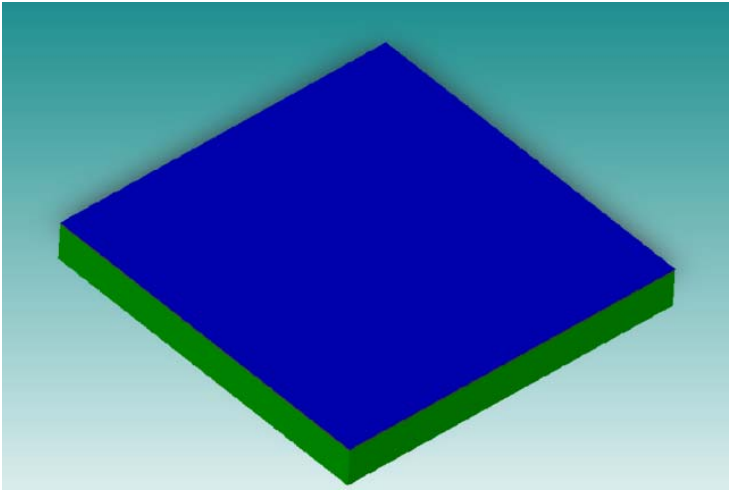

(c)Nitride 1

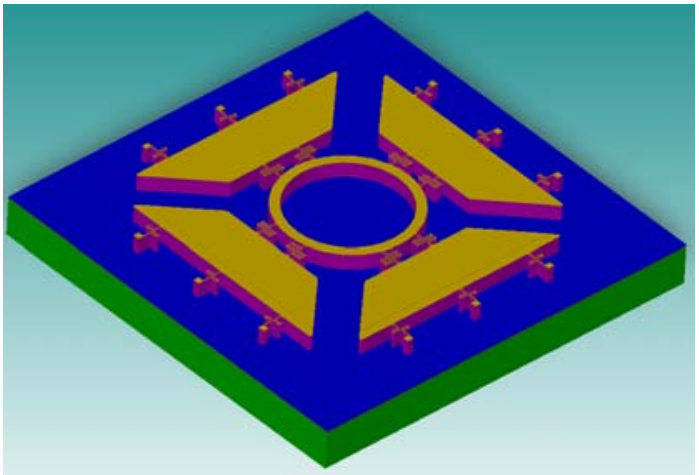

(e) Gold plating over nickel structure

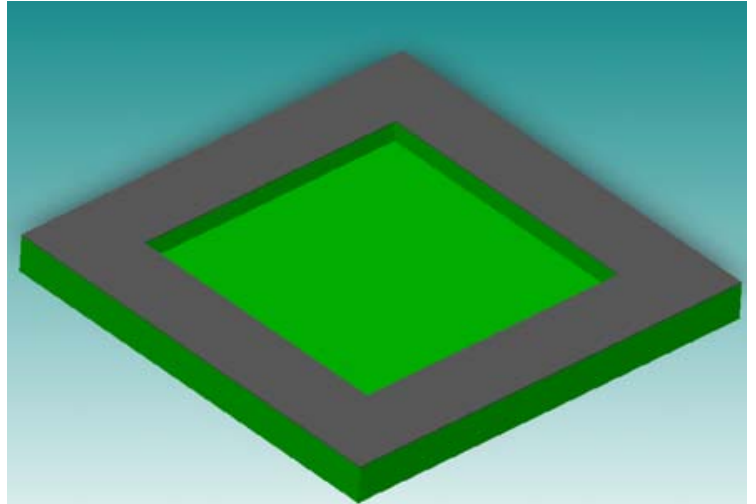

(b)Sacrificial layer (Oxide1)

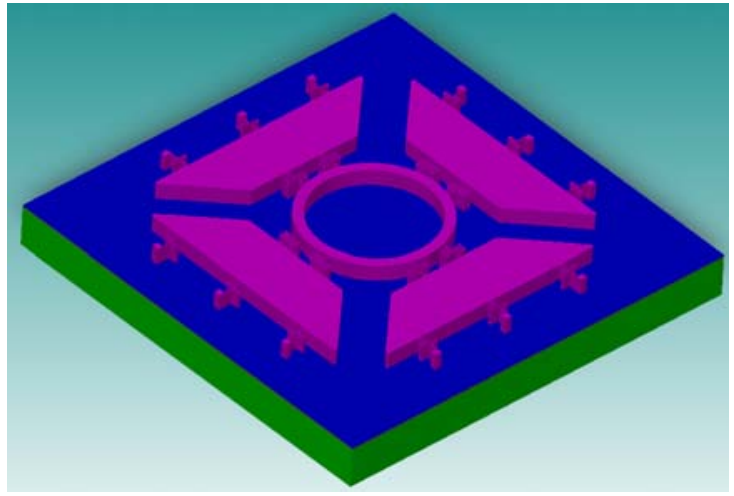

(d)Electroplated Nickel Structure

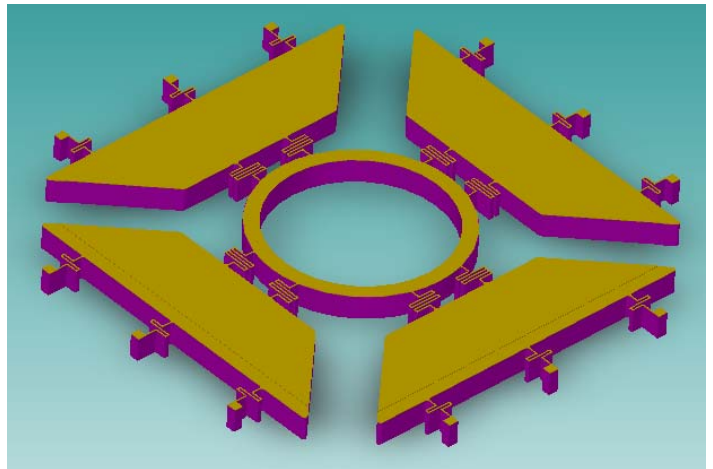

(f) 3D model of final structure

Figure 5.3: MetalMUMPs Simulation of Creating 3D Model using CoventorWare software 


\subsection{Prototypes}

Prototype has been fabricated using commercial process call MetalMUMPs. MEMSCAPE has provided this service of fabricating the prototype. The visual testing of the prototype was performed using a Zygo microscope. The image of the prototype under Zygo is shown in Fig 5.4

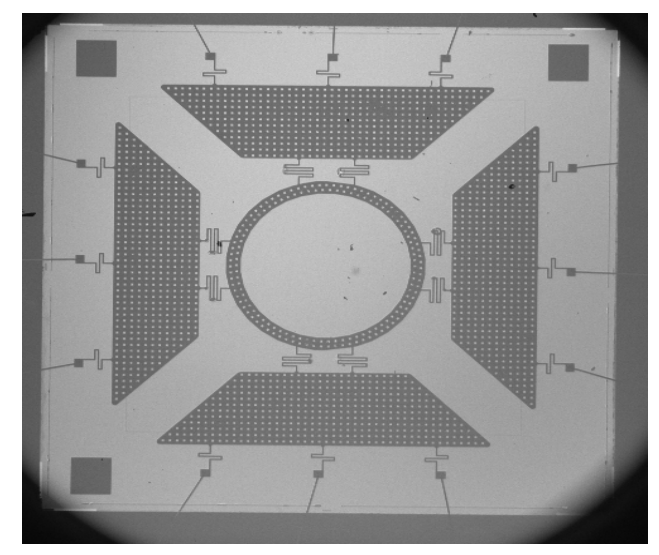

Figure 5.4: Protoype under Microscope Zygo

Another student experimentally verified that the designed actuator is capable of producing displacement under electromagnetic interaction.

\subsection{Micromirror Fabrication}

The mirror can be fabricated using polished wafer and coated with a thin layer of gold. The dimension of the mirror $(3.2 \mathrm{mmin}$ diameter and $0.2 \mathrm{~mm}$ in thickness) is large which makes it possible to fabricate using simple and mature microfabrication process. The mirror will be bonded with the central ring of the actuator using adhesive material. It has been tried by another student that the MEMS magnetic actuator can survive during the bonding operation. This verifies the proposed solution of separate fabrication of mirror and actuator plate and bonded them together. 


\subsection{Solenoid Fabrication}

The solenoids are not fabricated in this thesis. It can be fabricated using the parameter developed in the Chapter 4.5. 


\section{Chapter 6: Conclusion}

\subsection{Conclusion}

It is found that the designed actuator can achieve 370 micron displacement at static mode while the displacement achieved by existing MEMS FTIRS at static mode is 54 micron are presented. Two microfabrication processes have been used to develop the translating mirror mechanism. Commercial MetalMUMPs process is used to fabricate the magnetic actuator moving part whereas the micromirror plate is fabricated separately using silicon wafer to achieve a high surface quality. The actuator moving part and the micromirror plate are assembled together to realize both large displacement and high surface quality. The performance of the MEMS FTIRS based on the translation micromirror designed in this thesis has been predicted to be higher than existing designs. The stress has been analyzed for the moving part of the actuator. Dynamic analysis has been conducted for the moving part of the actuator along with the micromirror plate. The first resonant frequency is $158.65 \mathrm{~Hz}$, which is much higher than the FTIRS working frequency. The prototype of the actuator moving part has been fabricated using MetalMUMPS process.

\subsection{Contribution}

- Designed a novel large displacement micro electromagnetic actuator.

The actuator can provide large displacement through interaction with magnetic field. The actuator has the capability of minimizing the tilting. The actuator is bonded with micromirror plate which is separately fabricated. Two microfabrication processes are used to achieve both large displacement and high surface quality.

- Prototypes are fabricated. 
Prototypes are fabricated using a commercial micromachining process MetalMUMPs. A visual testing of prototypes is done using a microscope and found the fabrication is successful.

- Calculated and simulated the displacement of the actuator, which is 370 micrometers.

- Dynamic analysis has been conducted for the moving part of the actuator along with the micromirror plate, and ensured that the translating micromirror has high enough resonant frequency.

- The actuator fixed part, i.e., the solenoids has been designed.

\subsection{Future Work}

- Optimizing the actuator moving part to achieve larger displacement

- Assembling the micromirror plate with the actuator moving part

- Fabricating the solenoids

- Assembling all components to build the complete MEMS FTIRS 


\section{References:}

[1] R. D. Robertson, E. A. Holmes, and W. G. M. Vanlaar. Integrating Systems: Creating a Continuum of Care. Proceedings of the 12th International Alcohol Interlock Symposium. Ontario, Canada, October 17-19, 2011. Ottawa: Traffic Injury Research Foundation, 2012.

[2] Mto.gov.on.ca. Impaired Driving - Ignition Interlock. [online] Retrieved from: http://www.mto.gov.on.ca/english/safety/impaired/interlock.shtml [Accessed: June 2013].

[3] Google.ca. Alcohol interlock device - google search. [online] Retrieved from: https://www.google.ca/search?es_sm=93\&espv=210\&q=alcohol+interlock + device $\&$ tbm $=$ is ch\&cad=h [Accessed: June 2013].

[4] V. A. Saptari and K. Youcef-Toumi. Sensitivity analysis of near infrared glucose absorption signals: towards noninvasive blood glucose sensing. Proceedings of SPIE. Optical Techniques and Instrumentation for the Measurement of Blood Composition, Structure, and Dynamics, 4163:45-54, 22 November 2000.

[5] R. Smith and F. K. Fuss. Theoretical analysis of interferometer wave front tilt and fringe radiant flux on a rectangular photodetector. Sensors, 13 (9):11861—11898, 2013.

[6] P. Hariharan. Basics of interferometry. Academic Press, California, USA, 1992.

[7] Biocenter.helsinki.fi. Home page of the molecular biophysics group. [online] Retrieved from: http://www.biocenter.helsinki.fi/bi/biophys/methods_ftir.html [Accessed: June 2013].

[8] P. R. Griffiths and J. A. Haseth. Fourier transform infrared spectroscopy (2nd ed.). Hoboken, NJ: Wiley-Interscience, 2007.

[9]Mmrc.caltech.edu. Index of /FTIR. [online] Retrieved from: 
http://mmrc.caltech.edu/FTIR/FTIRintro.pdf [Accessed: June 2013].

[10] C. P. Sherman. Infrared spectroscopy. In: Settle AF, Handbook of Instrumental Techniques for Analytical Chemistry. Prentice Hall; Har/Cdr edition Chapter 15, 1997.

[11] D. Bell, T. Lu, N. Fleck and S. Spearing. MEMS actuators and sensors: Observations on their performance and selection for purpose. Journal of Micromechanics and Microengineering, 15 (7):153-164, 2005.

[12] J. W. Park, J-H Yang and H. Kim, "A large-deflection high-force micro electromagnetic hydraulic latex membrane actuator for fluid manipulation in micro channels", In Proceedings of the IEEE $24^{\text {th }}$ International Conference on Micro Electro Mechanical Systems (MEMS), pp. 1209-1212, 23-27 January 2011.

[13] C. Liu. Foundations of MEMS. Upper Saddle River, NJ: Pearson/Prentice Hall, 2006.

[14] R. Ghodssi and P. Lin. MEMS materials and processes handbook. Berlin: Springer, 2011.

[15] B. C. Smith. Fundamentals of fourier transform infrared spectroscopy. Boca Raton, FL: CRC Press, 1996.

[16] D. Reyes, E. Schildkrau, J. Kim, R. Connors, P. Kotidis and Cavicchio. A novel method of creating a surface micromachined 3d optical assembly for MEMS-based miniaturized FTIR spectrometers. Proceedings of SPIE, 6888, MEMS Adaptive Optics II, 68880D, February 2008.

[17] A. Tortschanoff, A. Kenda, M. S. Kraft, T. Ner, H. Schenk and W. Scherf. Improved moems-based ultra-rapid fourier transform infrared spectrometer. Proceedings of SPIE, 7319, Next Generation Spectroscopic Technologies II, 73190H, 28 April 2009. 
[18] O. Ferhanoglu, H. R. Seren, S. L. Uttjohann and H. Urey. Lamellar grating optimization for miniaturized fourier transform spectrometers. Optics express, 17 (23):21289—21301, 2009.

[19] F. Lee, G. Zhou, H. Yu and F.S. Chau. A MEMS-based resonant-scanning lamellar grating fourier transform micro-spectrometer with laser reference system. Sensors and Actuators, 149 (2):221-228, 2009.

[20] H. L. Kung, S. R. Bhalotra, J.D. Mansell, D. A. B. Miller and J. S. Harris. Standing-wave fourier transform spectrometer based on integrated MEMS mirror and thin-film photodetector. IEEE Journal of Selected Topics in Quantum Electronics, 8 (1):98-105, Jan/Feb 2002.

[21] E. R. Deutsch, D. Reyes, E. R. Schildkraut and J. Kim. High-resolution miniature FTIR spectrometer enabled by a large linear travel mems pop-up mirror. Proceedings of SPIE, 7319, Next-Generation Spectroscopic Technologies II, 73190J, 28 April, 2009.

[22] C. Drabe, T. Klose, H. Schenk, A. Wolter and H. Lakner. A large deflection translatory actuator for optical path length modulation. Proceedings of SPIE, 6186, MEMS, MOEMS, and Micromachining II, 618604, 21 April, 2006.

[23] C. Solf, J. Mohr, and U. Wallrabe. Miniaturized LIGA Fourier transformation spectrometer. Proceedings of IEEE Sensors, 2: 773-776, 22-24 October 2003.

[24] C.Ataman, H. "Urey, S. Isikman and A. Wolter. A MEMS based visible-NIR fourier transform microspectrometer. Proceedings of SPIE, 6186, MEMS, MOEMS, and Micromachining II, 61860C, 21 April, 2006. 
[25] Y. Kim, N. Kim, J. Kim, S. H. Lee, Y. Kwon and Y. Kim. 60-ghz full MEMS antenna platform mechanically driven by magnetic actuator. IEEE Transactions on Industrial Electronics, 58(10):4830—4836, 2011.

[26] F. S. Chau, Y. Du and G. Zhou. A micromachined stationary lamellar grating interferometer for fourier transform spectroscopy. Journal of Micromechanics and Microengineering, 18 (2):025-023, 2008.

[27] O. Manzardo, H. P. Herzig, B. Guldimann, C. Marxer and N. F. De Rooij. New design for an integrated fourier transform spectrometer. Proceedings of SPIE, 4178, MOEMS and Miniaturized Systems, pp. 310-319, 22 August 2000.

[28] H. L. Kung, S. R. Bhalotra, and D. A. B. Miller.Edward L. Ginzton Laboratory. Compact standing-wave transform spectrometer based on integrated MEMS mirror and thin-film photodetector. Stanford University, Stanford, CA 94305-4085.

[29] H. L. Kung, S. R. Bhalotra, J. D. Mansell and D. A.B Miller, A. Compact fourier transform spectrometer based on sampling a standing wave. IEEE/LEOS International Conference on Optical MEMS, pp.19-20, 21-24 August 2000.

[30] K. A. G. Prakash, S. Dhabai, E. Bhattacharya, and S. Bhattacharya. Design and fabrication of a micro-mirror for spectroscopy. Proceedings of SPIE, 7594, MOEMS and Miniaturized Systems IX, 75940X, 16 February 2010.

[31] A. Kenda, S. L"Uttjohannb, T. S. Krafta, A. Tortschanoffa and A. Simonb. A compact and portable IR analyzer: progress of a moems FT-IR system for mid-IR sensing. Proceedings of SPIE, 8032, Next-Generation Spectroscopic Technologies IV, 80310O, 12 May 2011. 
[32] C. Liu and Y. W. Yi. Micromachined magnetic actuators using electroplated permalloy. IEEE Transactions on Magnetics, 35 (3):1976-1985, May 1999.

[33] H. Guckel, T. Earles, J. Klein, J. Zook and T. Ohnstein. Electromagnetic Linear Actuators With Inductive Position Sensing For Micro Relay, Micro Valve And Precision Positioning Applications. The 8th International Conference on Solid-State Sensors and Actuators, 1995 and Eurosensors IX. Transducers '95, 53 (1):324-327, 25-29 June 1995.

[34] W. N. Sharpe,Jr., K. Jackson and G. Coles. Young's modulus and fracture strength of three polysilicons", Materials Science of Micro Electromechanical System (MEMS) Devices III,MRS Proceedings 657:EE 5.5.1-6, 2001.

[35] Y. Li, A. G. Webb, S. Saha, W. W. Brey, C. Zachariah and A. S. Edison. Comparison of the performance of round and rectangular wire in small solenoids for high-field NMR Magnetic Resonance in Chemistry, 44 (3):255-262, 2006.

[36] M. Kraft, A. S. Kenda, T. Ner and H. Schenk. Mems-based compact ft-spectrometers-a platform for spectroscopic mid-infrared sensors. IEEE Sensor, pp. 130-133, 26-29 October 2008.

[37] R. Tilburgs. Design and realization of a solenoid for a Robocup kicking device, 22, DCT 2006.120, Internal Report, Technische Universiteit Eindhoven, 2006.

[38] I. Cho, K. Yun, H. Lee, J. Yoon and E. Yoon. A low-voltage two-axis electromagnetically actuated micromirror with bulk silicon mirror plates and torsion bars. The $15^{\text {th }}$ IEEE International Conference on Micro Electro Mechanical Systems (MEMS), pp. 540-543, 24 January 2002 . 
[39] I. Cho, and E. Yoon. A low-voltage three-axis electromagnetically actuated micromirror for fine alignment among optical devices. Journal of Micromechanics and Microengineering, issue. 8, $19085007,2009$.

[40] H. J. Cho and C. H. Ahn. A bidirectional magnetic microactuator using electroplated permanent magnet arrays. Journal of Microelectromechanical Systems, 11 (1):78-84, February, 2002.

[41] C. Lee, Z. Chen, H. Chang, C. Wen and C. Cheng. Design and fabrication of novel micro electromagnetic actuator. Symposium on Design, Test, Integration and Packaging of MEMS/MOEMS, pp. 52-55, 9-11 April 2008.

[42] R.C. Hibbeler. Mechanics of materials $\left(6^{\text {th }}\right.$ Ed). Upper Saddle River, N.J.: Pearson Prentice Hall, 2005

[43] D. J. Sadler, T.M. Liakapoulos and C. H. Ahn. A universal electromagnetic microactuator using magnetic interconnection concepts. Journal of Microelectromechanical Systems. 9 (1):460-468, December 2000.

[44] B. Liu, D. Li, X. Yang and X. Li. Design and Fabrication of a Micro Electromagnetic Actuator. 1st IEEE International Conference on Nano/Micro Engineered and Molecular Systems, NEMS '06, pp. 353-356, 18-21 January 2006.

[45] E. M. Purcell. Electricity and magnetism. Cambridge: Cambridge University Press, 2011.

[46] A. Cowen, B. Dudley, E. Hill, M. Walters, R. Wood, S. Johnson, H. Wynands and B. Hardy. MetalMUMPs design handbook. MEMSCAP Inc.; Durham, NC, USA: 2009. 